\title{
Peer-to-Peer Overlay Techniques for Vehicular Ad hoc Networks: Survey and Challenges
}

\author{
Abdelkader Ilyes Ameura,*, Abderrahmane Lakas ${ }^{\mathrm{b}}$, Yagoubi Mohamed Bachir ${ }^{\mathrm{a}}$, \\ Omar Sami Oubbatic \\ ${ }^{a}$ Computer Science and Mathematics Laboratory, University of Laghouat, Laghouat, Algeria. \\ ${ }^{b}$ College of Information Technology, UAE University, Al Ain, UAE. \\ ${ }^{c}$ LIGM CNRS-UMR 8049, University Gustave Eiffel, Marne-la-Vallée, France.
}

\begin{abstract}
Vehicular ad hoc networks (VANETs) provide an effective technology for vehicles communicating while on the road. Vehicles are organized in an ad hoc networks offering support for both safety-critical messaging, multi-hop entertainment applications, and connection to internet services through Road Side Units (RSUs). Sharing data between connected vehicles can help commuting people exchange travel information, and allows content delivery and entertainment applications using Peer-to-Peer (P2P) techniques. Despite the widely documented benefits of $\mathrm{P} 2 \mathrm{P}$ techniques, there are a number of challenges faced when deployed in such a highly dynamic environments as VANETs. Such difficulties include the broadcast storm problem, network partition, and the temporal network fragmentation to name few. These issues are principally due to the high mobility of vehicles and thus to the constantly changing topology of the network. In this paper, we present a survey of the existing P2P approaches in the context of VANETs including a comprehensive survey of the various problems related to data sharing in VANET using P2P techniques, along with their proposed solutions from the perspective of data access, information lookup, routing, and message management. In this study, we provide a classification of the studied approaches in terms of network overlays, information structures, and network coding. To differentiate between the discussed approaches, we include a comparative study based on the crucial parameters and identify and discuss the open research challenges in P2P techniques. To the best of our knowledge, this survey constitutes the first attempt at a comprehensive analysis of P2P approaches in VANETs, which describes more appropriately the state-of-art of this specific topic of research.
\end{abstract}

Keywords: VANET, P2P, Information dissemination, Overlay, Clustering, DHT.

\footnotetext{
*Corresponding author

Email addresses: ak.ameur@lagh-univ.dz (Abdelkader Ilyes Ameur), alakas@uaeu.ac.ae (Abderrahmane Lakas), m.yagoubi@lagh-univ.dz (Yagoubi Mohamed Bachir), omar-sami.oubbati@univ-eiffel.fr (Omar Sami Oubbati)
} 


\section{Introduction}

Peer-to-Peer (P2P) is a promising research area, and especially in wireless networks. P2P systems are characterized by their distributed control and their hierarchical organization, which allow establishing robust communication. Indeed, in a traditional P2P system, each node can play the role of a client and server. The major challenge of $\mathrm{P} 2 \mathrm{P}$ systems is the design and deployment of robust distributed systems consisting of a set of participants that can be personal computers (PCs), laptops, sensors, or mobile nodes (e.g., vehicles or drones) [1]. As a result, some P2P systems could have a million active participants connected at the same time. Moreover, such systems do not require any centralized administration, which provides three main advantages: (i) the scalability and the starting condition of the network, (ii) the ability of aggregation and the use of computing resources and data, and (iii) fault tolerance and intentional attack resistance due to the distributed nature of P2P systems. Therefore, all these advantages make P2P systems well suited to both long-term storage and heavy computations. P2P systems can address many data-sharing issues. One of these issues is the lookup problem, i.e., how to find any given data item in an extensive P2P system. This issue reflects the conception complexity of $\mathrm{P} 2 \mathrm{P}$ networks. To solve this issue, many researchers proposed the use of a Distributed Hash Table (DHT) for the lookup process [2]. Each data is identified by a unique key included in the DHT, and therefore the proposed algorithms have to find the responsible node for storing this data based on its identifier. For this purpose, each node has to keep the information (e.g., IP or MAC addresses) related to its neighbors forming overlays to both store and retrieve data keys.

\subsection{Motivation}

The development of P2P architecture in recent years has also been adopted within Vehicular Ad hoc Networks (VANETs), which is a logical structure established over a physical network. In VANETs, there are hundreds or thousands of vehicles equipped with wireless interfaces and GPS, which are cooperating and sharing data, services, and computational power, e.g., traffic information, environmental data, multimedia, and messages [3]. Also, the greatest goal of VANETs is to provide information and services by the communication between vehicles and infrastructure using the paradigm of vehicle-to-vehicle (V2V), vehicleto-infrastructure (V2I), Vehicle-to-Pedestrian (V2P), over a cellular network (V2N), and especially the Vehicle-to-Everything (V2X) communications $[4,5,6]$. The primary goal of V2X communications is to ensure secure and comfortable transportation, besides reducing pollution and gas costs. Therefore, it uses all cited network connections and even the new vehicle-to-cloud (V2C) communication technologies [7, 8]. VANETs variant communications strive to solve traffic security, transport efficiency, and entertainment application issues [9]. In this last decade of significant research and progress in VANETs, extensive knowledge has been gained on designing VANETs, and many techniques and protocols have been proposed. For instance, $5 \mathrm{G}$ cellular communications remedy V2X network limitations and give (5G-V2X) birth $[10,11]$. 5G network performance allows V2X communications to handle vehicular safety to avoid collisions in vehicle platooning, remote driving, and advanced driving. In addition, it improves analyzing the performance of Collective Perception service, 
redundancy, and the information freshness of extended sensors [12, 13]. Unlike VANET security applications that require information from the vicinity of vehicles, many applications can use this information on a broader environment, such as parking guidance systems, navigation systems, driving comfort applications, and sharing applications. In the latter type of applications, the information is collected and shared in a wide and cooperative manner. The type of this information can take various forms [14], such as videos, music, traffic information, etc. However, information dissemination over big networks, such as VANETs, is a great challenge.

Among the most critical constraints, we distinguish the broadcast storm problem[15]. This problem happens when many vehicles want to communicate simultaneously, which also causes different other issues, such as network overhead, network congestion, packet collision, and additional MAC delays. An important number of proposals [16, 17, 18, 19, 20] tried to solve these problems. However, they neglected many other issues related to VANET characteristics, such as the high mobility of nodes. The latter issue can cause another critical constraint that is the network partition problem, which occurs when a vehicle wants to communicate in a sparse network (i.e., there are no vehicles in the vicinity). The network partition is a challenging problem because the vehicles are randomly distributed in the area, which leads to a challenging issue in data dissemination over the network. After all, the messages cannot easily reach a part of the network [21]. The last constraint is the temporal network fragmentation problem, which is induced by network changing topology caused by the highly dynamic topology of these networks. For instance, a solution has been proposed by authors in [17] to overcome this problem, where their main idea is to both maintain and discover alternative routes, but it amplifies the broadcast storm problem. To solve all these issues and constraints, many $\mathrm{P} 2 \mathrm{P}$ approaches are proposed with the aim of sharing information between all vehicles all over the network and overcoming the different limitations of VANETs.

\subsection{Organization of the survey}

To draw a full picture of $\mathrm{P} 2 \mathrm{P}$ systems, this work is organized based on eight sections handling the following details(see., Figure 1):

- Section 2 describes the P2P networks, their characteristics and classifications, and the main challenges in this kind of network.

- Section 3 introduces the concept of VANETs and describes their design, their characteristics, and their routing protocols.

- Sections 4 provide a comprehensive and critical review of the proposed P2P techniques over VANETs, present a taxonomy of overlay independent, structured overlays and unstructured overlays schemes in VANETs, provide a detailed description of the majority of $\mathrm{P} 2 \mathrm{P}$ contributions in VANET proposed across the literature along with their illustrative figures and identify and discuss the main challenges in this area, respectively. 


\section{Survey Organization}

Peer-to-peer (P2P) Networks 1). We provide a comprehensive introduction of $P 2 P$ networks, and their and classifications depending on various criteria, such as storage, processing, and information sharing.

2). We highlight the different mechanisms, characteristics, and the main challenges in this kind of network.

\section{P2P techniques for VANET}

1). We present a taxonomy of overlay independent structured overlays and unstructured overlays schemes in VANETs.

2). We provide a detailed description of the majority of P2P contributions in VANET proposed across the literature along with their illustrative figures.

Open issues and future research directions

1). We summarize the different future research directions, which we think are relevant to our work.

2). We provide a brief definition for each research direction and how the chemistry between P2P overlay and these last ones can be benefic.

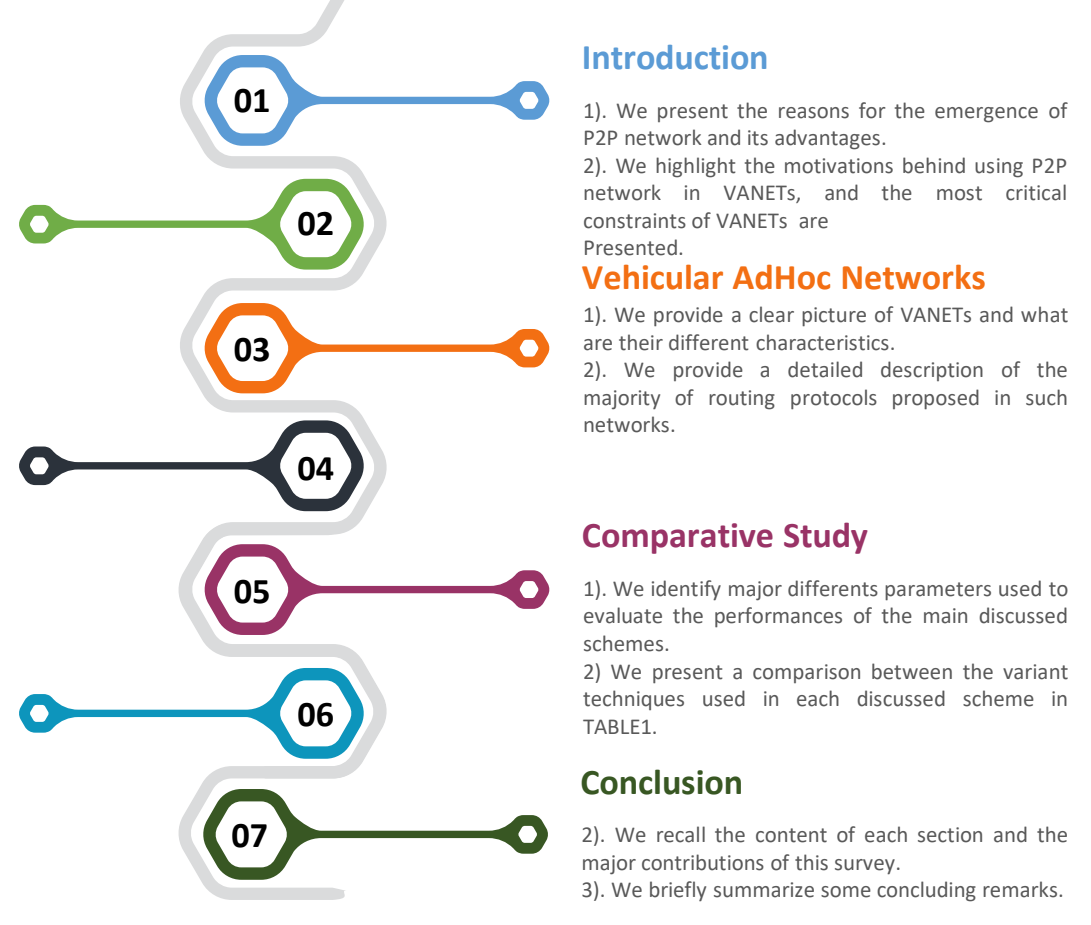

Figure 1: Organization of the survey 


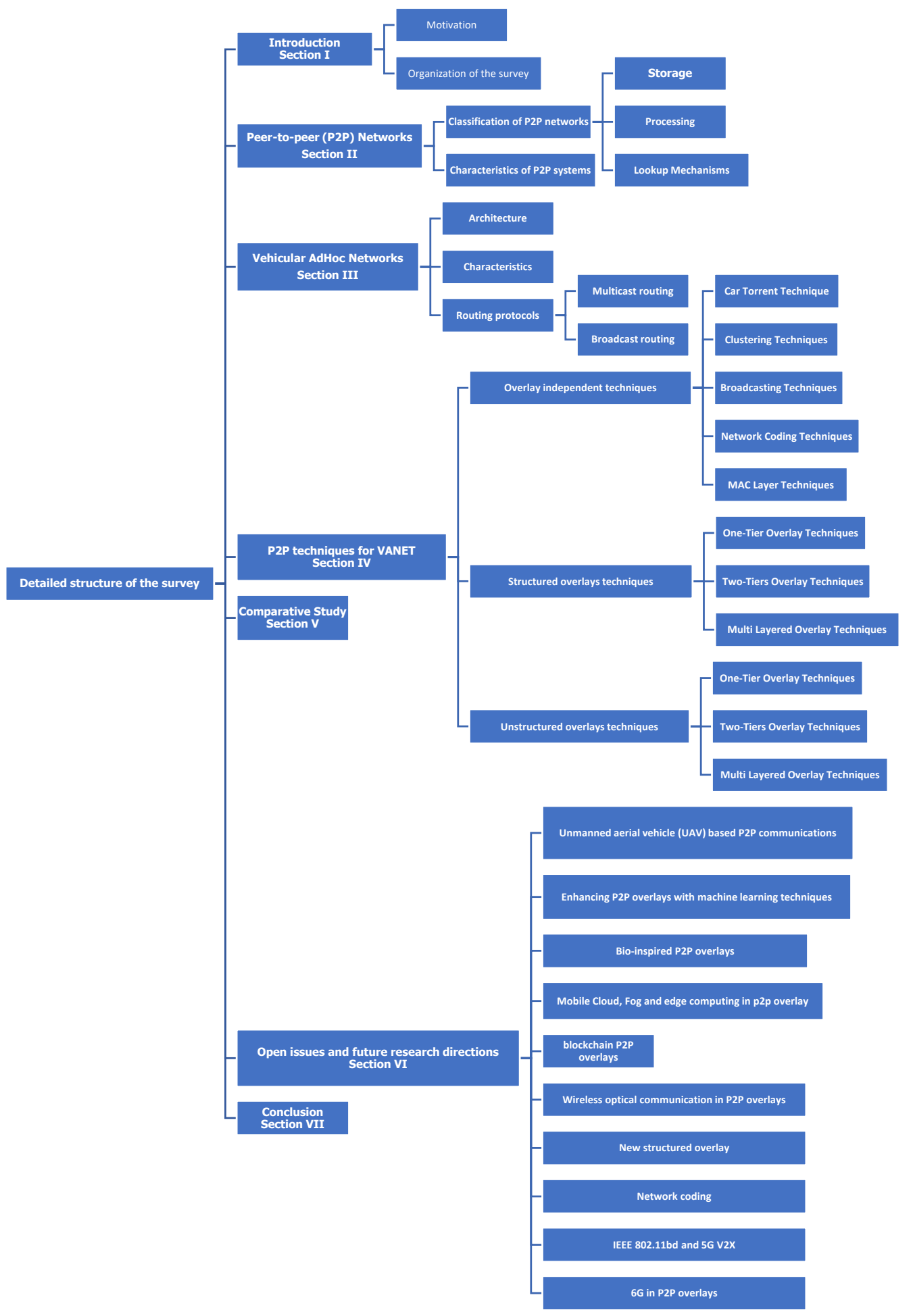

Figure 2: Detailed structure of the survey 
- Section 5 compares and classifies the most recent works in this field.

- Section 6 presents several open issues and future research directions.

- Section 7 presents concluding remarks. Also, a brief summary of contributions is presented in this section.

For more clarity, we use the term peer, node, car, and vehicle interchangeably.

\section{Peer-to-peer (P2P) Networks}

P2P systems comprise self-organized equal and autonomous entities (peers) aiming to share distributed resources in a given network [22]. P2P systems are virtual networks in the application layer with a specific overlay topology and supported by routing protocols. The overlay topology is defined in a way where the nodes are connected together, and the routing protocols define which nodes can exchange messages and share information and resources. The overlay topology and routing protocols have a key role affecting many properties of the system, such as performance, reliability, and scalability.

\subsection{Classification of P2P networks}

There are many classifications of P2P systems. In this section, these systems are classified into three categories depending on various criteria, such as storage, processing, and look up mechanisms.

\subsubsection{Storage}

In centralized systems, the central server is responsible for maintaining information about nodes and data where each peer has to communicate (i.e., sending queries) with the central server to have the data. In addition, this kind of systems is used by Napster [23] (see., Figure 4-A). The advantage of this centralized architecture is that it is simple, quick, and has an efficient information discovery. However, it has several vulnerabilities to be attacked (i.e., if the server is attacked, the system will be down), and several other drawbacks, such as the scalability restriction due to database sizes, the high number of queries to be responded, and the frequent periodic updates.

In decentralized systems, all nodes have the same functionality. Therefore, they act as servers and clients simultaneously. Chord [24] and CAN [25] are relevant examples of decentralized P2P systems (see., Figure 4-B). This kind of systems is highly scalable because there is no centralized aspect making them fault-tolerant to the loss of an important number of peers. The nodes have autonomous control over their contents. However, they have no quality of services along with joining and leaving problems.

The Hybrid architecture is divided into two designs: (i) Design with a central server and (ii) Design without a central server. In the design with the central server, the latter registers the users' list of the system and facilitates peer discovery. In addition, there are many peer servers (i.e., super peers or super nodes [26]) that memorize both information about users and content indexes of its peers. At the same time, they coordinate with the 


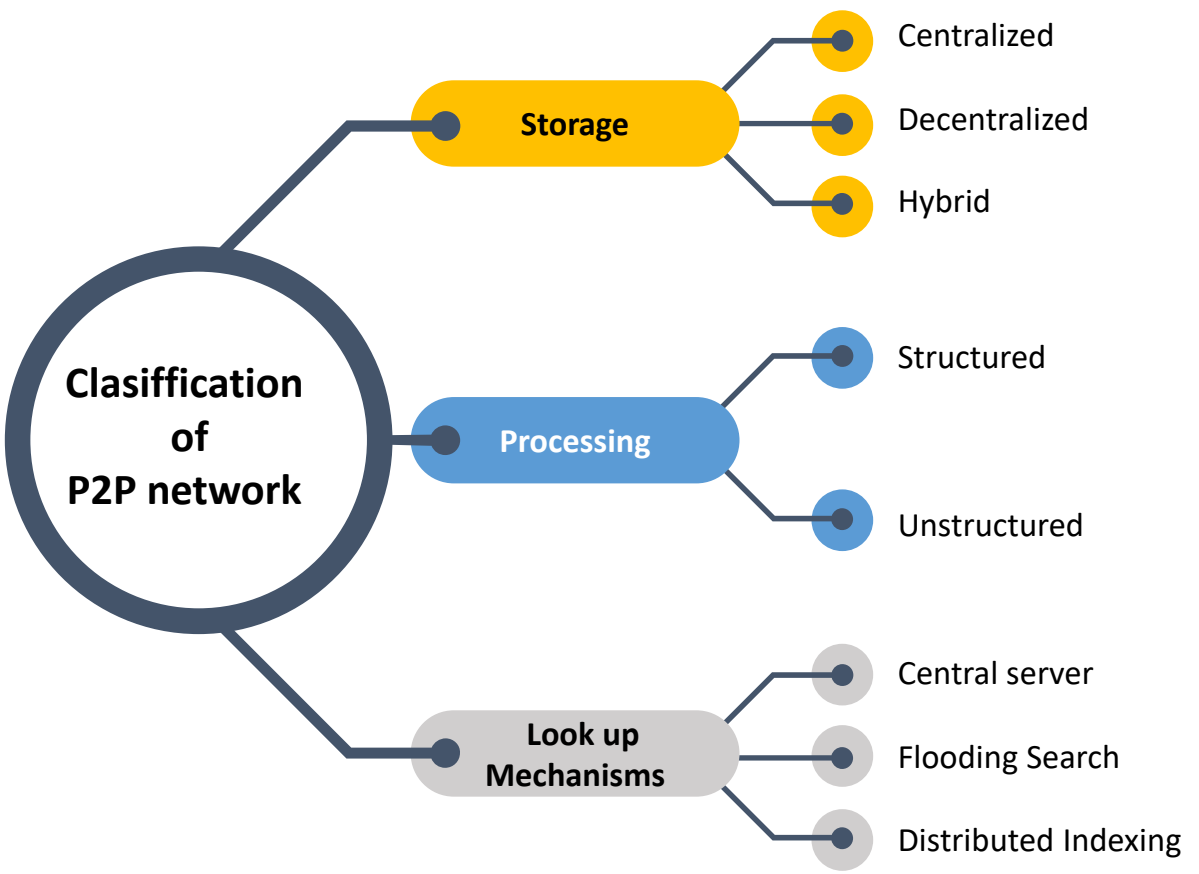

Figure 3: Classification of P2P networks 


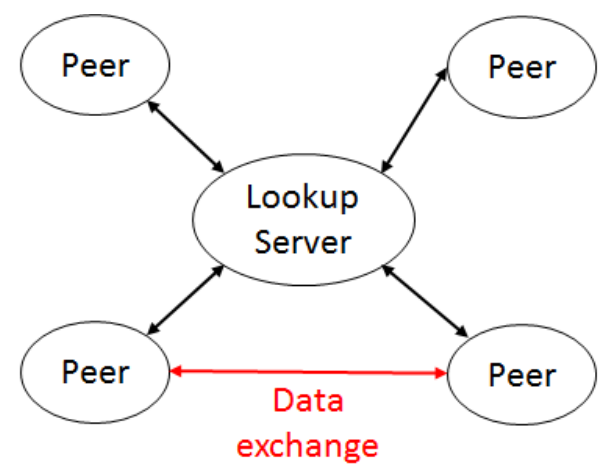

A- Centralized Architecture

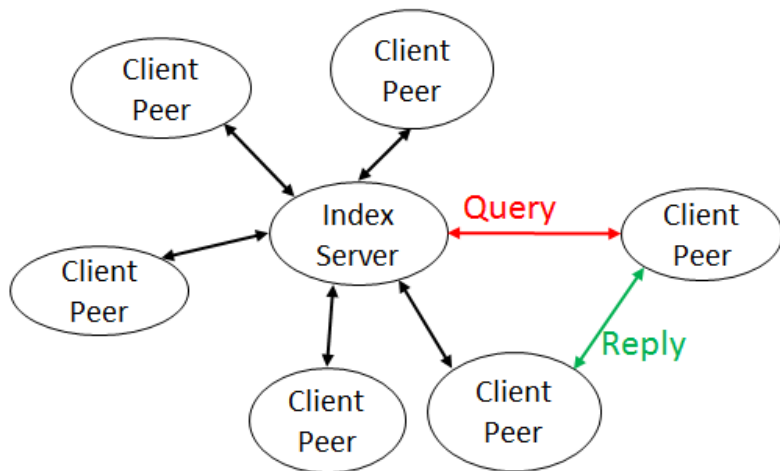

C- Hybrid Architecture with central server

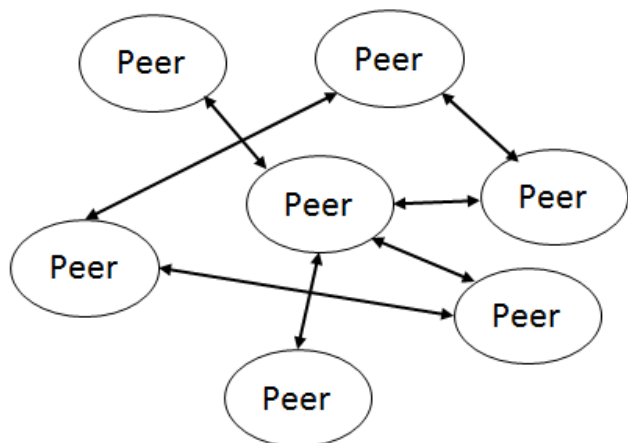

B- Decentralized Architecture

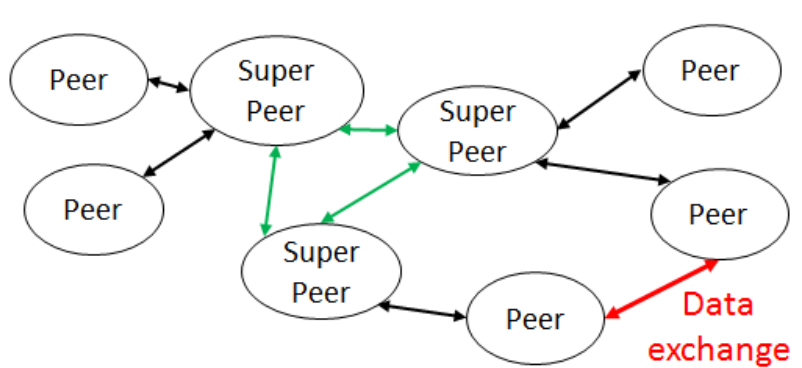

D- Hybrid Architecture without central server

Figure 4: Centralized Vs. Decentralized 
central server to manage their peers' lookup queries, since the queries are directly sent to super peers. This kind of architecture is used by Kazaa [27] (see., Figure 4-C). Moreover, super peers are elected depending on bandwidth and processing power, which allows the central server to provide new peers with a list of super peers that they can connect with. In the design without a central server, when a node wants to join the network, it has to contact the super-peer to validate its joining. If this newly entered node has a powerful processor, it can become a super-node, and then it forms a network with other super peers. This type of architecture is used by Gnutella [28] (see., Figure 4-D).

\subsubsection{Processing}

Structured P2P networks are used to distribute keys' space between peers, and each one has to be responsible for a specific key space partition, so that it has to store all information related to this key space partition. This helps a peer to find content using only the content key.

In unstructured systems, the peers are connected in an ad hoc manner so that they are able to operate in a decentralized way, even if they are within a centralized structure. In addition, the content placement is independent from the overlay topology. However, structured systems are based on a controlled overlay, where peers are indexed, and data is both indexed and distributed among peers based on mapping rules between the content and peer indexes (e.g., DHT).

\subsubsection{Lookup Mechanisms}

There are three basic strategies to store and retrieve data in $\mathrm{P} 2 \mathrm{P}$ systems: (i) Central server, (ii) Flooding Search, and (iii) Distributed Indexing. In the first strategy, the central server maintains the current locations of data items. If a peer joins the overlay, it has to share its contents and resources and it has to send this content's information to the central server so that the central server can respond to requests (i.e., data locations) of other nodes directly. This mechanism is used by Napster [23] (see., Figure 5(a)).

In the second strategy, to retrieve an element in this mechanism, the node must request all participating nodes in the network if necessary, which means that the nodes do not share information about their contents, so this lookup technique broadcast a request for an item among the peers of the system. If a node receives a request, it forwards this request to other nodes until finding the content or exceeding a predefined number of hops (Time to Live TTL). In addition, some broadcast mechanisms replicate the content in the network, to avoid data loss and improve lookup delay. Gnutella [29] is a good example of this mechanism type (see., Figure 5(b)) .

The third strategy, such as DHT is used in structured overlays because it provides a global view of distributed data in the network. Each node and content (data) has a key, and this key can be provided by a hash function, where, each node is responsible for some data depending on the DHT type. Therefore, by hashing data items, any node can find the content through a small number of hops. For more details, the following Figure 5(c) shows how Chord [24] is designed. 


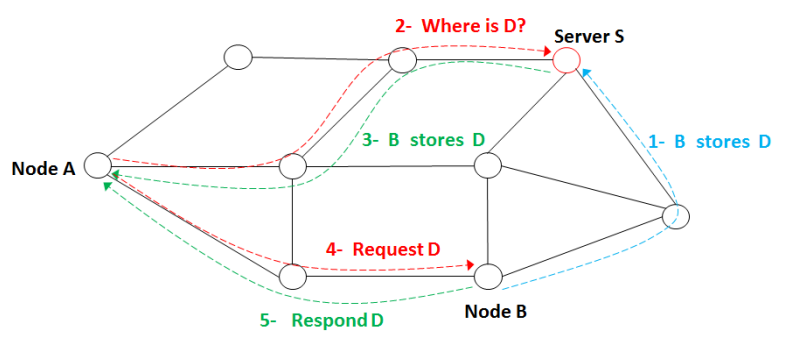

(a) Central Server

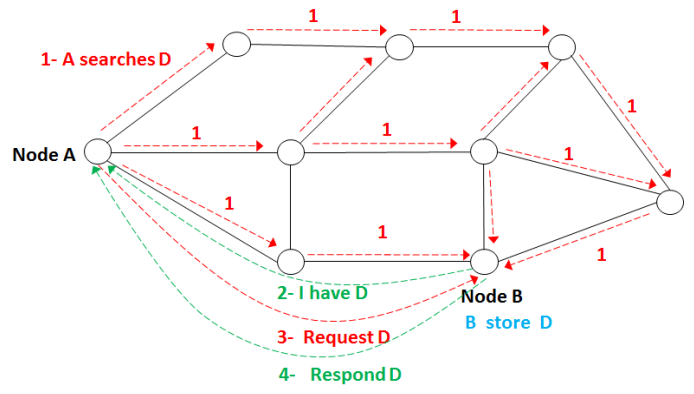

(b) Flooding Search

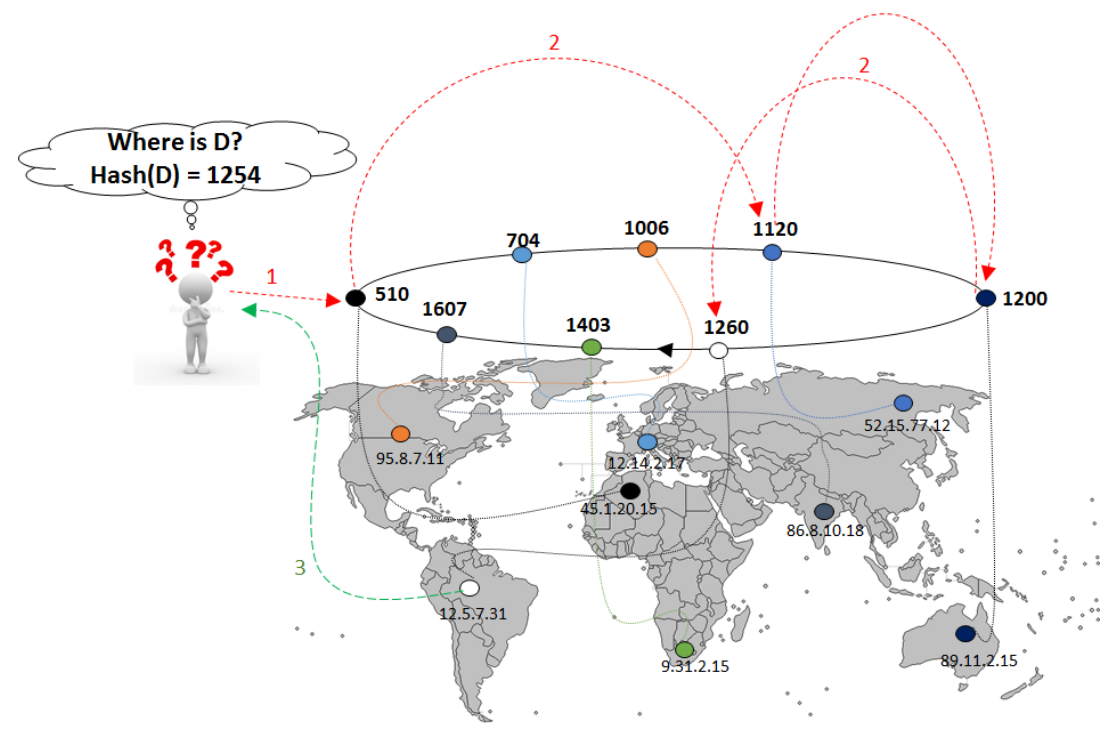

(c) Distributed Indexing

Figure 5: Lookup Mechanisms 


\subsection{Characteristics of P2P systems}

$\mathrm{P} 2 \mathrm{P}$ systems have the following characteristics:

1. Distributed resources like bandwidth, storage, and processing power.

2. Peers are connected within a network in a distributed manner, except in certain cases such centralized structures.

3. Each peer has a dynamic IP address, because of its multiple connections and disconnections to the network.

4. Content identifier is derived from its name using a hash function.

5. In general, peers directly communicate with each other to exchange content. However, there are some cases when peers have to transit through the central server.

6. Unlike other systems, P2P nodes act as clients and servers at the same time.

7. Peers have the same functionality, so they are fully autonomous.

\section{Vehicular AdHoc Networks}

In VANETs, the topology of the network changes frequently due to the high mobility of nodes. VANETs are autonomous wireless networks created between vehicles, where it is used both for safety driving application [30, 31, 32, 33, 34], for Traffic information systems (TISs), and for internet connection. In addition, they can collect and share important data used for route guidance and Intelligent Transportation Systems (ITSs) where the vehicles have short and medium range wireless communications. Consequently, vehicles are able to communicate, detect, and compute. In recent years, a new network known as Flying adhoc networks has emerged (FANET) $[35,36,37,38,39,40]$. It is a hybrid of UAVs and a VANET. UAVs can communicate directly or through intermediate nodes using the wireless medium [41]. For that reason, it differs from VANET in a few characteristics of VANET, especially in limited power, mobility, topology changes, and radio propagation.

\subsection{Architecture}

For a deep understanding of VANET architecture, it is important to study its different communication models, and this shows the full potential of vehicular communications. VANET is a highly dynamic network, vehicles have embedded on-board-units (OBUs) including wireless transmitter and receiver. Therefore, vehicles can communicate: (i) through some RSUs (V2R /V2I). (ii) with each other (V2V), (iii) with pedestrian (V2P), (iv) over a cellular network (V2N), and especially with everything (V2X) [4, 5, 6] (see., Figure 6). VANETs can be deployed in three different environments, such as urban, rural, and freeway/highway [42, 43, 44]. Each environment has its own mobility challenges. This is the reasons why there is an important number of research solutions $[45,46,47]$ trying to handle the different mobility issues. There are many mobility models that are classified into 


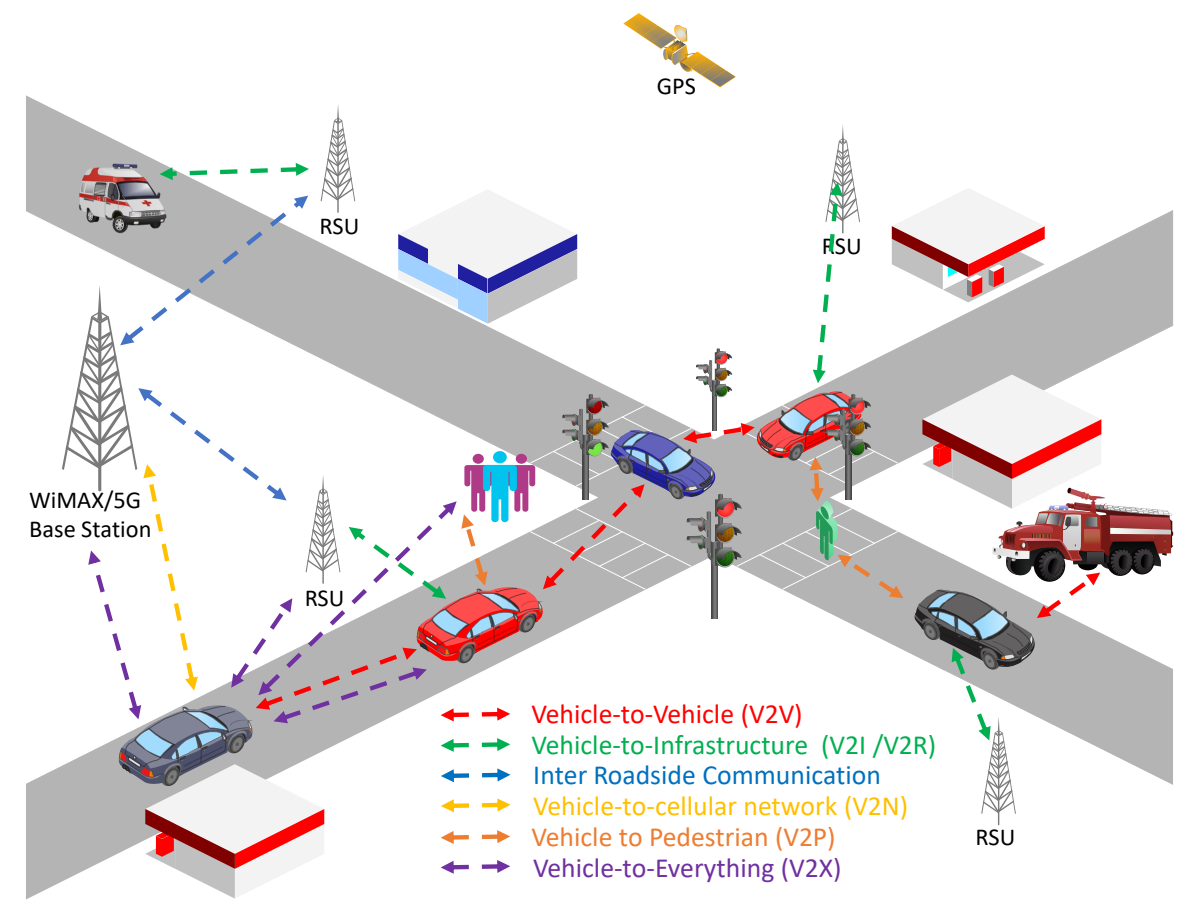

Figure 6: VANET Architecture

three categories based on many factors, such as acceleration, deceleration, lane changes, and human conduct[48, 49]:

1. Stochastic or random modelling,

2. Traffic or flow modelling, and

3. Trace modelling.

A high data rate is required for advanced VANET applications, such as cooperative collision warning, traffic management, and entertainment [50, 51]. As a result, the need for antennas that can handle several bands for communication in VANET applications is skyrocketing. Furthermore, bandwidth is critical for delivering efficient performance in terms of vehicle safety. New protocols such as IEEE 802.11bd (for Dedicated Short Range Communications (DSRC)) and 5G NR V2X (for Cellular V2X (C-V2X)) were born as a result of improved high reliability and low latency requirements. The $5.9 \mathrm{GHz}$ band (5.850-5.925 $\mathrm{GHz}$ ) is used for V2X features, with a bandwidth of $75 \mathrm{MHz}$, which is insufficient for today's demands. New bands with greater capacity in the sub $6 \mathrm{GHz}$ region will be delivered as part of the $5 \mathrm{G}$ rollout, allowing for better Cellular-V2X capability and superior performance. This necessitates a more significant number of communication devices, resulting in increased connection and high link dependability, which may be met by installing a large number of radio transceiver components. In addition, because it can receive signals from all directions, an antenna with more radiating components is likely to deliver a high-quality 
signal. Any antenna element in the system that fails to catch the intended signal can be seen with greater quality by another antenna element in the system [52], given that the antenna elements are isolated, as coupling between the components reduces performance and perhaps signal quality.

To avoid such effects, Multiple Input Multiple Output (MIMO) [53] systems that make use of numerous diversities are highly recommended. By combining many antenna components into a single module, a robust diversity scheme and better link reliability may be achieved. Many researchers have suggested numerous forms of antennas for VANET purposes throughout the years, including shark fin antennas. Despite their primary purpose, these antennas failed to meet the requirements for many antenna properties such as isolation and radiation pattern [54]. Slots in antenna design and the notion of fractals allowing broadband operation are two more strategies that have aided the implementation of planar antennas in V2X applications [55]. [56, 57] proposes a Penta band antenna for vehicle communication that excludes particular operating vehicular communication bands from the needed bands. The authors of [58] created a fractal antenna for vehicle communication that uses a compliant substrate, although it does not cover the LTE 42 band.

Planar antennas with MIMO implementation have recently demonstrated significant advantages over conventional antenna designs, as they address fundamental demands such as isolation, polarization diversity, spectrum efficiency, and enhanced signal-to-noise ratio. It also contributes to the high throughput needed in V2V and V2I situations [59, 60]. The isolation between close antenna parts is the most critical factor to consider while developing MIMO antennas. The millimeter wave (mmWave) [61, 62, 63, 64] band is used in 5G and other wireless communication networks. Although the massive bandwidth available in this band allows for unparalleled throughput, it also has a higher propagation loss than the bands below 6 Ghz. This property necessitates a full rethinking of the old communication paradigm, namely, omnidirectionality. Indeed, mmWave systems employ directed beams produced by antenna arrays, or beamforming, to provide appropriate received signal strength. By appropriately aligning the antenna components of the antenna array, beamforming concentrates power (both transmit and receive) in the desired direction.

\subsection{Characteristics}

VANETs have many characteristics as follows:

1. Frequent disconnections: Due to the high mobility of vehicles, the communication links cannot remain stable all the time.

2. Infrastructure access: Due to the high mobility of VANETs, communication infrastructures, such as RSUs, access points, and hotspots, can be deployed to maintain network connectivity and communication.

3. Computing Capacity: To send, receive, process, and store data, each node in VANETs has to be equipped with processing capabilities, storage ability, and energy capacity. Since they are equipped with rechargeable batteries, the energy of vehicles is not restricted. 


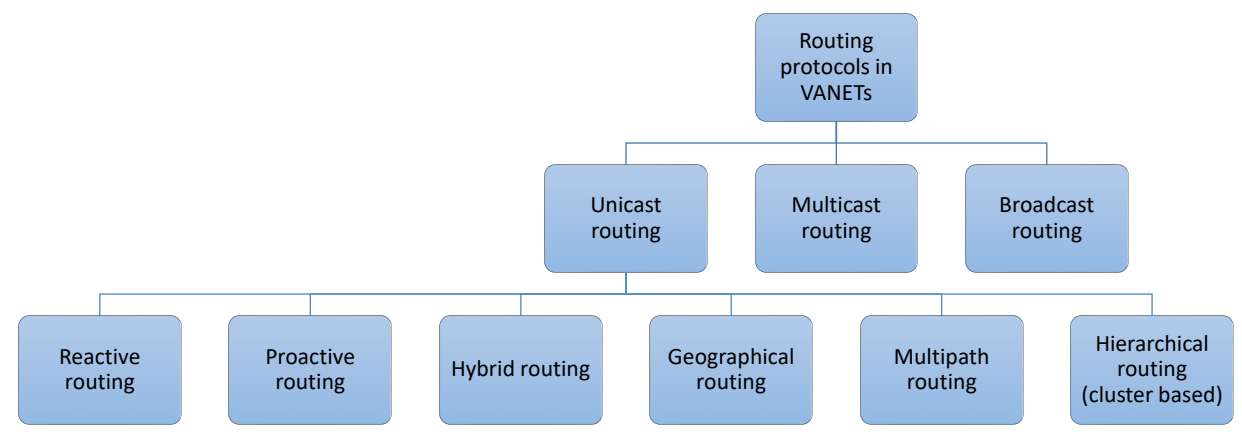

Figure 7: Taxonomic of VANET routing protocols

4. Geographical position: In VANETs, the majority of vehicles are equipped with a Global Positioning System (GPS) used to locate the vehicle.

5. Communication environments: The vehicles move in various places, such as cities, highways, and rural zones.

6. Dynamic density: VANETs have a dynamic density depending on the location where they are deployed in (i.e., highway, city, or urban) and the moment (i.e., rush hours or nights).

7. Delivery delay constraints: There are several security applications (e.g., alert dissemination) in VANETs, which help to avoid accidents. In this kind of applications, the delay of transmission has a significant impact on the dangerous situation avoidance.

8. On-board sensors: Each vehicle is equipped with various sensors that ensure data gathering.

\subsection{Routing protocols}

There are several routing studies [43, 65, 66, 67], which illustrate many challenging issues to design a routing protocol while taking into account the high mobility of vehicles and the changing topology of the network. Many MANET routing protocols have been adapted for VANETs, but the problem is the high message dissemination delay. Therefore, the challenge is how to find and maintain the optimal routing paths between peers in such networks. In the following, the VANET routing protocols are classified into seven classes (see., Figure 7): 


\subsubsection{Unicast routing}

In this kind of protocol, the source node sends data to another single node via intermediate relays that can use store and forward schemes. This helps to minimize the network overhead and the delivery delay. This category of routing protocols can be classified into six sub-categories:

Reactive routing. In reactive approaches, such as Adhoc On-demand Distance Vector (AODV) [68], Dynamic Source Routing (DSR) [69], AODV with Predicting Node Trend (AODVPNT) [70], AODV with Multi-RREP (AODV-MR) [71], Reactive Routing Protocol for VANETs (RRPV) [72], and Speed Based on Demand Vector Link Routing Protocol (SODV) [73], the route request messages are sent if there is a need, thus if a node wants to send a message to a specific destination, it broadcasts a Route Request Messages (RREQ) to their neighborhood, then each node that receives this message must forward it until it reaches their destination. Finally, the destination node sends back the message with the route using unicast communication. In addition, there are some reactive protocols based on Artificial Intelligence (AI) and Reinforcement Learning (RL), such as Point to Point Ad-Hoc On-Demand Vector (PP-AODV) [74], Portable Fuzzy constraints Q-learning AODV (PFQ-AODV) [75], Adaptive Routing Protocol based on RL (ARPRL) [76], Reliable Self-Adaptive Routing (RSAR) [77], Q-Learning-based AODV for VANET (QLAODV) [78], Practical and Intelligent Routing protocol for VANET (PIRP) [79], and Heuristic Q-learning based VANET Routing (HQVR) [80].

Proactive routing. In these protocols, each node has a routing table that must be periodically updated with the new route information, and this information must be broadcasted to their neighbors using Hello messages. Therefore, if a node requires to send a message to the destination, it uses the routing table to reach its goal. But unfortunately, these sorts of techniques consumes the network bandwidth. There are a great number of Proactive protocols like Destination Sequenced Distance Vector (DSDV)[81], Optimized Link State Routing (OLSR)[82], Global State Routing (GSR) [83], and Pheromone-Based Vehicle to Vehicle (PBV2V) Routing [84].

Hybrid routing. In these types of protocol, designers try to exploit the benefits of proactive and reactive routing. Therefore, if the destination node is not far from the source node, the source node uses a routing table (Proactive Routing) to achieve its destination, else it uses reactive routing. There are many variants of hybrid routing protocols detailed in [85] like: Zone Routing Protocol (ZRP) [86], Distributed Dynamic Routing (DDR) [87], Distributed Spanning Tree (DST) [87]. In addition, there are some Hybrid protocols based on AI and RL, such as RL-Based Hybrid Routing protocol (RHR) [88], Q-Learning and GRID-based routing Protocol for vehicular Adhoc networks (QGRID) [89], RL assisted Zone based VANET Routing Protocol (RLZRP) [90], Adaptive Data Collection Protocol using RL (ADCPRL) [91], and VANET Routing using Deep RL Technique (VRDRT) [92].

Geographical routing. With the aim to improve routing performance and reduce the control overhead, these algorithms use the geographic positions (Global Positioning System 
(GPS)) of all the vehicles in routing processes, so the nodes in the network must be aware of each others geographic position. There are many examples like Geographic Landmark Routing (GLR) [93], Location-Aided Routing (LAR) [94], Distance Routing Effect Algorithm for Mobility (DREAM) [95], Analytical Hierarchical Process (AHP)-Based Multimetric Geographical-Routing Protocol (AMGRP) [96], Named Data VANET Protocol (NVP) [97], Multimetric Next-Hop Vehicle Selection for Geocasting in Vehicular Ad-Hoc Networks [98], VANET Routing Based on Real-Time Road-Vehicle Density [99], Adaptive Geographical Routing Based on Quality of Transmission for Urban Vehicular Networks (AGQOT) [100], Greedy Curvemetric Routing Protocol (GCRP) [101], Greedy Perimeter Stateless Routing (GPRS)-Modified (GPSR-M) [102], Maxduration-Minangle GPSR (MM-GPSR) [103], Connectivity-Aware Intersection-Based Routing (CAIR) Protocol [104], Distance and Signal Quality Aware Routing (DSQR) [105], Q-learning based Traffic-Aware Routing protocol (QTAR) [106], Position-Based Q-learning Routing (PBQR) [107], and Q-learning based Load Balancing Routing (Q-LBR) [108].

Multipath routing. Unlike other routing protocols, these protocols attempt to find all possible paths between source and destination using reactive or proactive routing. Due to the highly dynamic nature of the VANET, these algorithms are divided into three processes: route discovery, route maintenance, and traffic allocation. Caching and multipath routing protocol (CHAMP) [85], Split Multipath Routing (SMR) [109], and Adhoc On-demand Oultipath Distance Vector routing (AOMDV) [110] describe in detail the three processes.

Hierarchical routing (cluster based). According to clustering techniques and an associated addressing scheme, these protocols build a logical hierarchy of nodes in the network. The nodes at a high level can be used to improve the connectivity scalability and the efficiency of the routing. But the major disadvantages of these algorithms are the increasing size of the routing tables and control packet overhead in the big networks. Hierarchical landmark routing (H-LANMAR) [111], Hierarchical State Routing (HSR) [85], and Core-Extraction Distributed Ad hoc Routing (CEDAR) [112] are an example of Hierarchical Routing protocols. Moreover, there are a number of Hierarchical routing based on AI and RL, such as Agent Learning-based Clustering VANET routing Algorithm (ALCA) [113], Context-aware Unified Routing for VANETS (CURV) [114], Reinforcement Learning-based Routing protocol for Clustered VANETS (RLRC) [115], RL and Game Theory based VANET Routing (RGVR) [116], and Reinforcement Learning routing in Software Defined Vehicular Routing (RL-SDVN) [117].

\subsubsection{Multicast routing}

In contrast to unicast protocols, multicast techniques deliver data to a group of nodes via multi-hop communications. This type of techniques is used with the aim to improve the packet delivery delay. Also, it uses clustering to ensure intra and extra cluster communications, but the cluster head selection is an additional issue. There are various multicast protocols, such as RObust VEhicular Routing (ROVER) [118] and Cluster-Based Directional Routing Protocol (CBDRP) [119]. 


\subsubsection{Broadcast routing}

Broadcast techniques are one of the first routing techniques used in VANETs [118]. . Basically, they use a flooding technique in the case where the message is needed to be sent to the node that is outside the communication range. This routing technique can ensure the message delivery, but it consumes bandwidth, congests the network, and increases the probability of packet collisions. To discover the route to the destination, several broadcast techniques, such as BROADCOMM [120] and the Nth-Powered P-persistent Broadcast protocol (NPPB) [121] can be deployed.

\section{P2P techniques for VANET}

In the aim of exploring the existing P2P methods in VANET, this section has been divided into three categories: overlay independent techniques, structured overlays techniques and unstructured overlays techniques.

\subsection{Overlay independent techniques for VANET}

In this subsection, we develop the first P2P techniques class in VANET that tries to overcome the limitation of such a network without P2P overlay improvement.

\subsubsection{Car Torrent Technique}

One of the first examples of P2P techniques in VANETs is Car Torrent [122]. It is a Java application that uses AODV and it is responsible for discovering and maintaining routes, It uses AODV to send Gossip messages and files, these applications are composed of:

1. A user interface,

2. A File Splitter: Responsible for splitting the file into a lot of pieces and combine them in the reception,

3. CarTorrent File Manager: responsible for tracing the parts of the file, it maintains the peers list and the hops number of each piece, it updates his information in each reception, and It selects the closest rarest pieces.

4. Send Gossip Thread: Sending Gossip messages from the client or the queue (others Gossips) depending on probabilities parametrized in the client program,

5. Receive Gossip Thread: if the received Gossip message is from the client, the message is deleted. If not it will be transmitted to CarTorrent File Manager and it will be treated and saved in the queue,

6. Listen Thread: it is triggered if there entrants connexions, and transmit these connexions to the Receive Packet Thread.

7. Receive Packet Thread: There to incoming messages:

(a) Data request: send the demanded data to the other client. 


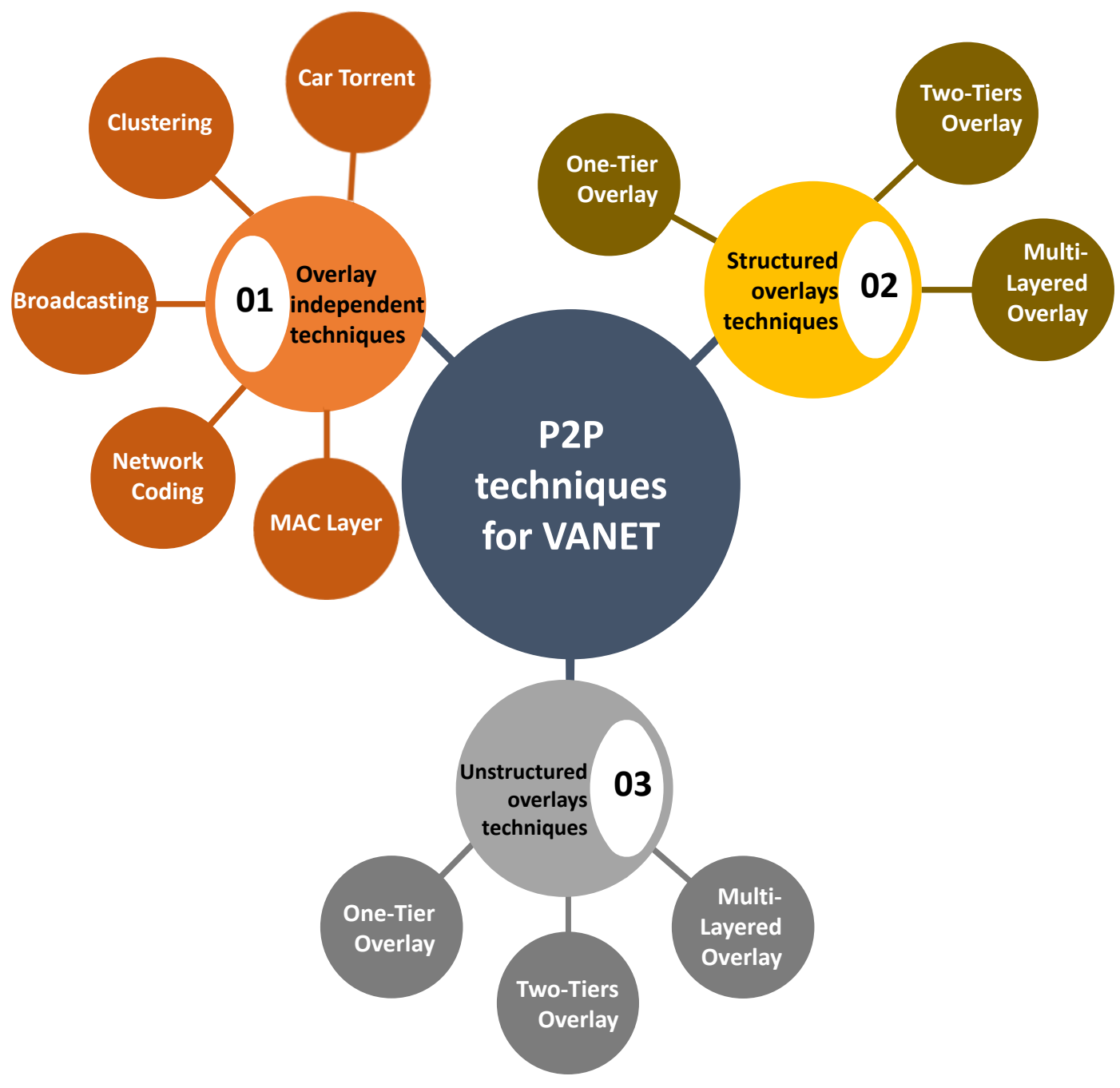

Figure 8: Taxonomy of P2P techniques for VANET 
(b) Data: saved in the local space of the client and combined b the File Splitter.

It is an interesting real-world application for sharing files. However, if there are many nodes, performance will suffer. However, this application does not use a list of peers who can have the file or the parts to void the disconnection problem (to detect the absence of files or pieces).

\subsubsection{Clustering Techniques}

One of the first examples of P2P techniques is presented in [123], to avoid congestion the authors use the warning (safety) messages in urban and highway zones, the peers are organized as a cluster (Peer Space, PS) according to common benefits and there are two ways of clustering (Cluster Based Organization and Peer Centered Organization), for sharing the information, this approach uses a mediation mechanism. The P2P resource mediation mechanism is the concept of distributed hash tables(DHT's)[124]. However, this approach does not allow a peer in one PS to inform peers in another PS, thus cannot be utilized in highways.

Another clustering technique proposed by C. Caballero-Gil et al [125]. This technique showed that a clustering technique designed can optimize the network load in the case of dense networks, the cluster is managed by the cluster head (leader), and the vehicles in the same group communicate via public and private keys, the Group stages are:

- Group detection: the node detects if there are a lot of neighbors,

- Group election: if there are many neighbors, the node must select one leader to join his group,

- Group creation: if there is not a leader, the nodes must create a group,

- Group membership: if a node leaves a group and the network is dense, it must join another

- Group life: if the network is sparse, the group must be scattered.

After the clustering, the message management inside groups is detailed in the algorithm in [125]. This paper defines the comprehensive process that nodes must follow in order to manage groups. This technique reduces network traffic and reduces packet loss. It is a flexible system that can be used in both sparse and dense networks. However, the simulation duration is quite short, it is inefficient in sparse networks, and if the network is particularly dynamic, the control messages can overload it, and we do not examine the relay node selection.

Another VANET communication model based on a P2P system that has been proposed in the literature tries to address blind spots and hot spots issues. The RSUs will no longer be necessary services in this study since the vehicle may be used as data relays. The degree distribution, as well as the agreement procedure, are used to designate the relay nodes. The performance parameters of a traditional VANET and a P2P network in a VANET have been 


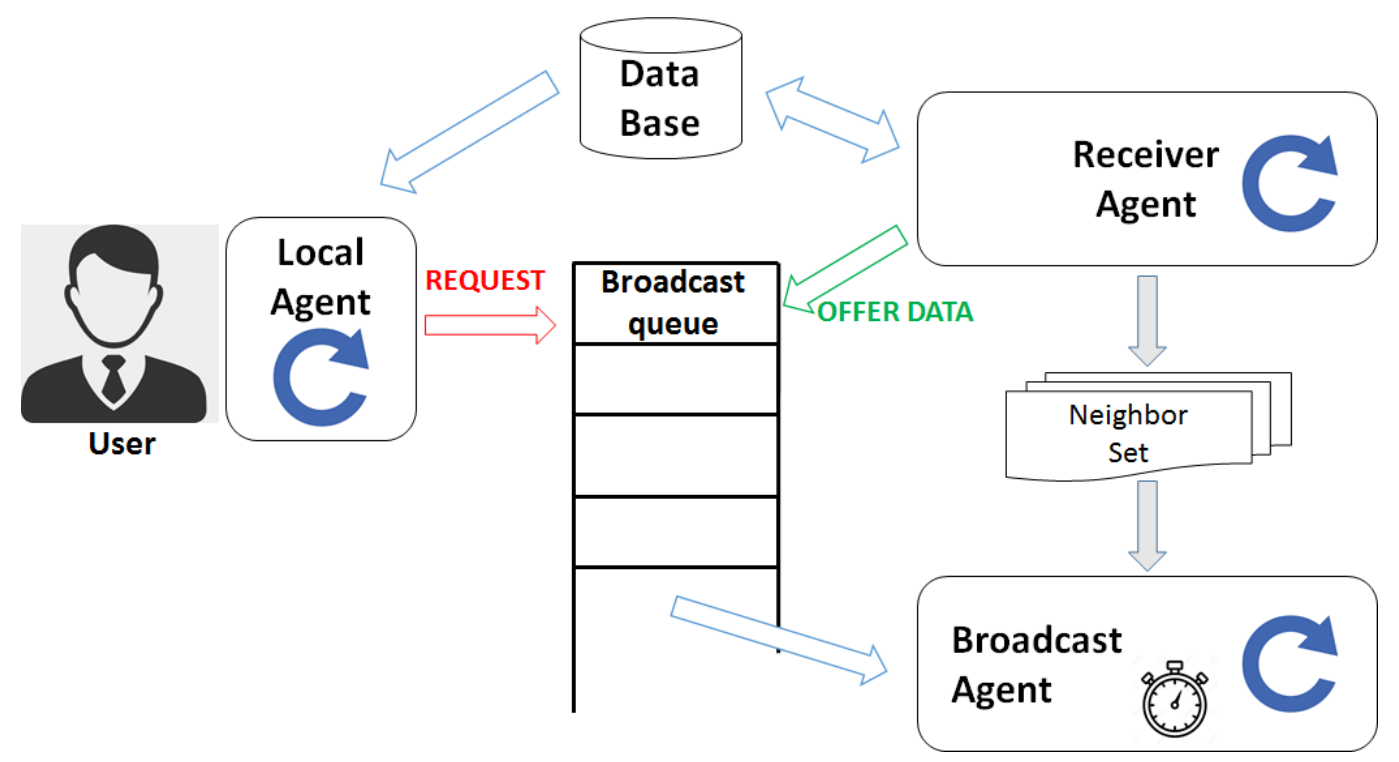

Figure 9: Design of a collaborative system based on epidemic broadcast [129]

compared using MATLAB simulation. As a result, the P2P network is demonstrated to be both cost-effective and real-time efficient. Nevertheless, in terms of self-organization, this view still has many issues [126].

In [127], an information-sharing technique uses V2V communications based on clustering and P2P-based Communication Framework for Geo-Location Oriented Networks (G-LocON) [128] to communicate with separated VANET groups through a mobile network.According to the authors, this proposal is efficient in packet arrival rates and dissemination delay, but it has not been tested in a dynamic network and the network relay on mobile communications.

\subsubsection{Broadcasting Techniques}

The design of a collaborative system based on the epidemic broadcast is another study proposed by C. Barberis et a.[129], this system can find a multimedia content from other vehicles or public internet servers because it uses multicast messages, like it is detailed in the scheme (see., Figure 9). This method aids in the sharing of content multimedia files in Vanet networks. It is effective when the network has a large number of RSUs. It can be utilized in any sort of area, and it can be used in various vehicle mobility models. However, because this technology overloads the network with multicasting messages (they employ a credit system for access medium sharing), it necessitates a large amount of memory to store the database (in each vehicle).

\subsubsection{Network Coding Techniques}

A lot of researches proof that network coding is an efficient method of networking, CodeTorrent [130], use the random linear coding system to share information on P2P networks in one-hop communication. It supposes that every file can be identified by fileID 


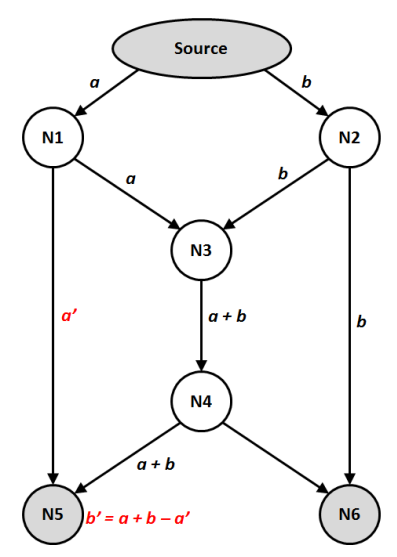

Figure 10: Network Coding [130]

and divided into $\mathrm{n}$ pieces, the seed node creates and diffuses the file descriptions to their one-hop neighbor. In this protocol, nodes exchange coded frames instead of file pieces and every time the seed node is invited to exchange coded frames, the node must generate a new frame. A node can know about a file if it receives the description of this file from his neighbors, but If a node finds the interesting file, it defuses the request with fileID, in the reception of this request, each node that has a piece or a coded frame that contain a piece of the requested file must respond with a new coded frame, to restore the complete file each node must collect $n$ coded frame linearly independent, every node must:

1. Broadcast periodically the description of the files contained in their coded frame to their neighbors with a low probability

2. Save all received frames (linearly independent of the others frames in the memory) even if it is not interested in this frame because it can help the neighboring nodes,

3. Use a little slot of random time (broadcast jitter) before broadcasting to avoid the collision.

In Codetorrent, the nodes work together to disseminate the coded bits via network coding. However, malicious nodes generate many problems, and specific propagation faults occur (incorrect frames can influence all the coded frames after linear combining).

\subsubsection{MAC Layer Techniques}

Two proposed techniques suggest a layer modification to improve P2P communications: The first one is presented by R. Xie [131] and Z.Y. Xie [132]. They have demonstrated that to ensure the streaming without stopping the video, we must use a P2P application to share multimedia content (e.g., video, music). For that reason, they conceive an Ad-hoc Stream Adaptive Protocol (ASAP), ASAP uses UDP in the transport layer to deal with route changes in VANETs, each video is divided into equal segments (with IDs), to join the network, the client node must broadcast joining request, it must choose the first server node, the intermediate (i.e., relay) node reacts like the real client node, the nodes must request the 


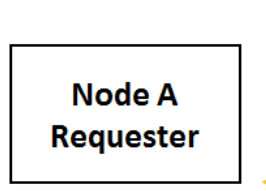

Orig. Requester $=$ self

Orig. Supplier $=$ B

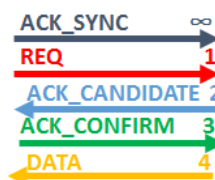

Orig. Requester $=\mathrm{A}$

Orig. Supplier $=\mathrm{C}$

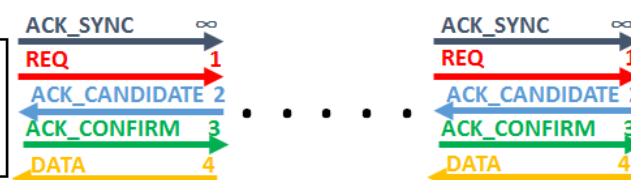

Orig. Requester $=\mathrm{B}$

Orig. Supplier $=$ D
Node D

Supplier

Orig. Requester $=\ldots$ Orig. Supplier $=$ Self

Figure 11: Ad-hoc Stream Adaptive Protocol (ASAP)

next segments before the playback buffer finish with the current segment and the ASAP can reuse an existing path many times (see., Figure 11).ASAP is better than Vanilla and ensures flexible playback in terms of efficiency and duration. It can stream again after a failure, and each relay node reconnects as if it were a client node. However, ASAP is dedicated to video streaming and nothing else, it can be accomplished through caching mechanisms, and it employs the first path rather than the best.

And the second one is proposed by M. S. Almalag et al. [133] and B. Nataraj et al [134]. In this technique, the authors have proposed an algorithm for P2P file-sharing over VANET using a new MAC protocol (i.e., TC-MAC) to improve inter-cluster communication. The network is divided into clusters, and each cluster has a cluster head $(\mathrm{CH})$. This protocol offers a dynamic assignment of TDMA slots. Like DSRC MAC, TC-MAC is divided into 1 Control Channel (CCH for safety/update messages) and 6 Service Channel (SCH) (see., Figure 12). Even if the cluster has a large set of vehicles, this technique may ensure file sharing and be used in different systems (TC-MAC use DSRC channels). However, this method only uses one-hop communication (MAC only), and the control messages management puts a strain on the network.

\subsubsection{Interference Evaluation Techniques}

The suggested method in [135] evaluates the impact of interference in P2P in VANET over independent and non-identically distributed Nakagami-m fading channels. The construction of a closed-form specification of the Probability Density Function (PDF) and overall Signalto-Interference-plus-Noise Ratio is used to derive P2P communication in this paper (SINR). Using the PDF, they get the mathematical closed-form formulation of the suggested system's Moment Generating Function (MGF). Furthermore, an Average Bit Error Rate (ABER) expression for $\mathrm{P} 2 \mathrm{P}$ communication is constructed under the impact of interference. So, this paper concentrates on the impact of interference on $\mathrm{P} 2 \mathrm{P}$ communications and provides essential mathematical expressions verified using Monte Carlo simulation, such as the PDF, MGF, and ABER. However, the other factors that influence the network are neglected.

\subsubsection{Security Techniques}

The different initiative [136] proposes an effective, secure routing technique, known as ESSPR, that combines searchable encryption with vehicle proxy encryption to maintain message security in vehicular P2P networks. The author has demonstrated that ESSPR 
can withstand packet processing, packet monitoring, replay attacks, wormhole attacks, and interference. The efficacy of ESSPR among malicious peers has proven that it is secure and efficient in terms of average fairness, delivery ratio, detection ratio, and average latency in vehicular P2P social networks. However, it must be tested with other kinds of attacks such as user-profiling attacks and liability attacks.

V. Hassija et al. [137] created a vehicular P2P network to ensure data tracking and communication security. In this paper, two models were suggested.

- Vehicle to RSU auction-based model: an agreement was reached in the car network using the IOTA [138] consensus algorithm.

- Vehicle to vehicle Nash bargaining model: An auction model was utilized to allocate a maximum amount of required bandwidth.

The Nash bargaining algorithm has been implemented in this proposition to offer automobiles with optimal functionality. In addition, a game-theoretical framework was used to simulate the connection between vehicles that provide and consume offloading resources. According to the simulation results, all vehicles in the network deliver superior Quality of Experience (QoE) and utility potential than their peers.

CSP2P [139] is a new security scheme for the urban P2P VANET network. It was created with the goal of maintaining a functional certification framework. In addition, an intelligent cooperative detection system is also presented, which detects routing threats via homomorphic encryption. The CSP2P system is offered as an intelligent black hole attack detection scheme with video segment protection to safeguard the Urban P2P VANET network. It differs depending on the type of attack, vehicle node authentication, and the video data's integrity and secrecy. This model can identify, prevent, and inform elementary and compound attacks in the urban P2P VANET environment depending on the simulation. Blacklists, on the other hand, can help with security and confidentiality.

\subsubsection{Safety Messages Techniques}

The VANET P2P data sharing system [140] aims to improve driver situational awareness and minimize road accidents. The proposed system includes numerous sensors that identify things on the road and send warning messages to other vehicles through RSUs. The development is made up of an embedded system that is installed in both the vehicles and RSUs. The suggested system focuses on the distance sensor, which provides distance and accuracy information. The statistics indicated that the suggested system could exchange information with automobiles and infrastructures to avert collisions and support safe driving.

\subsubsection{Priority Signal Control Techniques}

In [141], a newly signal-priority control model is designed, which serves as the foundation for a Multi-Modal Intelligent Traffic Signal System (MMITSS). The MMITSS is a USDOTfunded initiative that focuses on connected vehicle technologies to create a cooperative transportation network. Because of the priority control concept, even a signal controller may have a scalable vehicle priority policy. Furthermore, the priority control approach minimizes 
the number of failures and interruptions by limiting the adverse effects on vehicles. Two VISSIM simulation networks evaluate the MMITSS. The results show that a P2P network can enhance the reliability of transportation while decreasing harmful effects on other modes of mobility. The analyzed parameters are priority control, coordination, and fully operational control. Although, vehicle priority management was established without taking coordination or pedestrians into account.

\subsubsection{Software Defined Network (SDN) Techniques}

You, Z., et. al.[142] developed a novel SDN direction technique for a peer-to-peer (P2P) network. It is a cross-layer protocol in VANET that uses cross-layer parameters such as available bandwidth, link duration, and forwarding probability to decide the route from source to destination. This technique is intended for urban vehicle networks that can determine the best path between source and destination on a global scale. The given technique evaluates the optimum global pathways for SDN and OpenFlow controllers vehicle communication and reduces end-to-end delay significantly. As a result, routing based on route reliability decreases the risk of a connection disruption, resulting in a reduced rate of route interruption. A network simulator named OPNET was used to compare this technique with SDN-based geographic routing (SDGR) protocol [143], SDN-based vehicular ad-hoc on-demand (SVAO) routing protocol [144], and AODV, and evaluate the performance of the proposed design at various speeds and densities. The simulation shows that this protocol is efficient in packet drop ratio, overhead, and average end-to-end delay throughput.

\subsubsection{Streaming Techniques}

TDDV [145] is a VANET-based P2P information distribution system for critical deadline streaming. It is a distinct search approach for gathering information and looking for segments in a specific province simultaneously. It is split into three functions, as follows:

- A lookup function that obtains data distribution part information to decrease network overhead.

- A function that requests data prepares only enough data to fulfill the service's playback rate requirement.

- A function solves the problem of highly mobile hosts in VANETs by providing deadlinesensitive segment distribution.

The NS shows that TDDV is better than other mechanisms in admission rate, successful rate, control bytes per second, average route delay, route duration, and single hop delay. However, the connected link duration is calculated based on the assumption that vehicle speeds follow some specific distributions. 


\subsubsection{Delay Tolerant Networks Techniques}

The described idea in [146] comprises two significant contributions to P2P communication using a data distribution method:

- Rubinstein Bargaining-based game-theoretical pricing model: A relay node must charge a service forwarder node based on the remaining service level in this paradigm.

- P2P communication into the data-centric suggested method to improve content distribution: The suggested algorithm's performance was measured using the message delivery ratio, average message delivery latency, and overhead ratio.

According to the simulation, the suggested method decreased data delivery delays, mitigated node selfishness, lowered overhead, and boosted the message distribution ratio.

Finally, in the following diagram, we present a taxonomic tree of overlay independent techniques for VANET(see., Figure 12).

\subsection{Structured overlays techniques for VANET}

Structured overlays mean that the resources are not randomly divided in the network. So the nodes must use the DHT, this feature facilitates the data discovery process due to the limited number of hops to find the resources. In most cases, the nodes and the resources have the same ID space, and the resources are distributed in the nodes depending on a hash function. In what follows, we will study the most known P2P structured overlays over VANET, and we will discuss their topologies, lookup process, and their performance.

\subsubsection{One-Tier Overlay Techniques}

Rybicki J Et al [3] developed a novel distributed traffic information system based on P2P overlay and internet. They have detailed several $\mathrm{p} 2 \mathrm{p}$ traffic information problems and their solutions like:

- Fairness and scalability

- Bootstrapping

- Network aggregation

- Privacy and trustworthiness

- connectivity

This proposition uses Publish/subscribe DHT (see., Figure 13), and It is based on three tasks:

- Detection changes to trigger the notifications

- Subscription management 


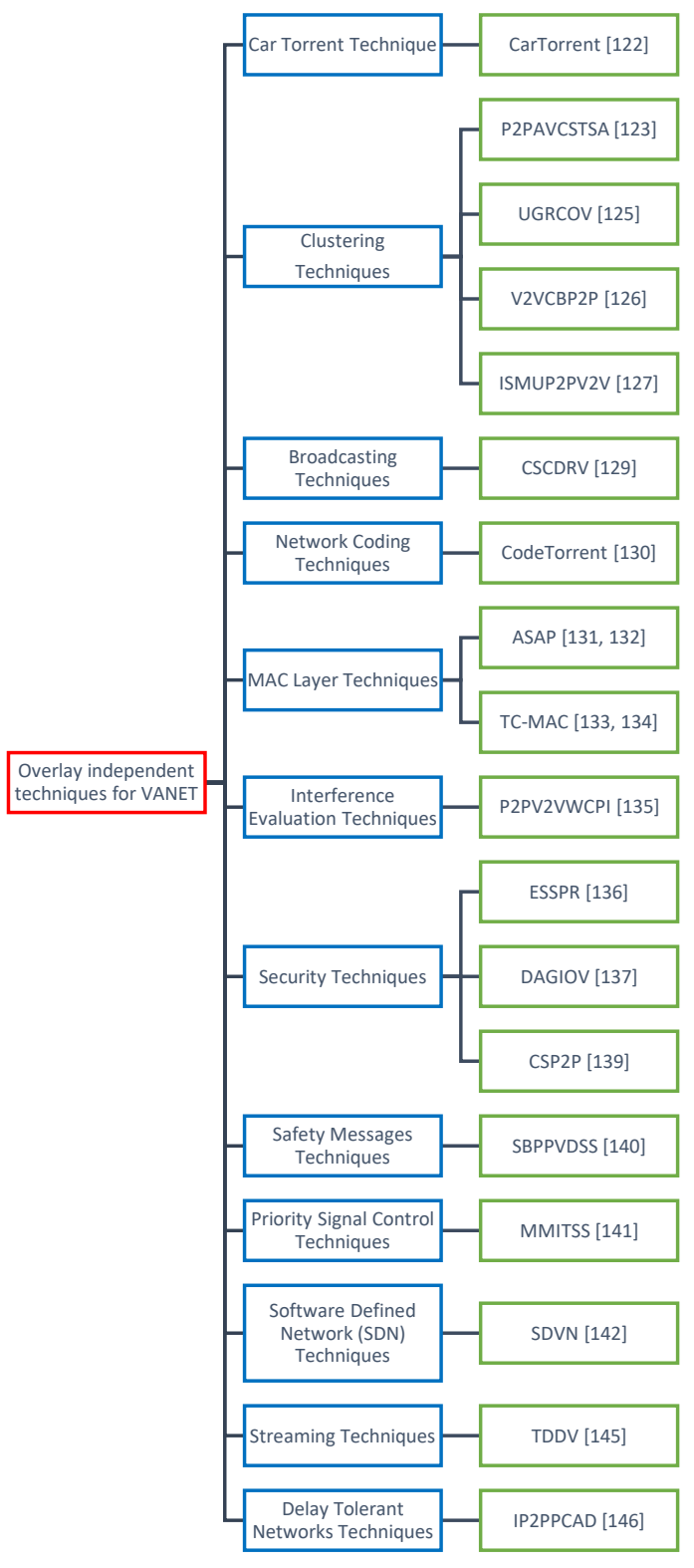

Figure 12: Taxonomic of overlay independent techniques for VANET 


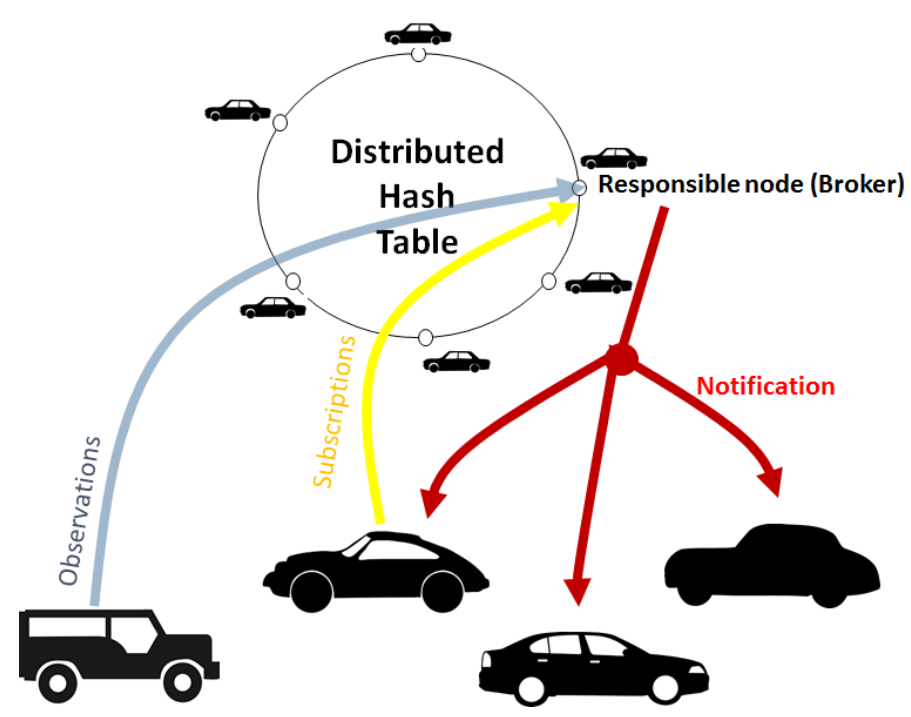

Figure 13: Publish/subscribe DHT [3]

- Information distribution

However, this approach does not allow a peer in one PS to inform peers in another PS, and it cannot be utilized in highways.

A new kind of VANET routing protocol that integrate P2P overlay in VANETs named Peer Computing based Adhoc On Demand Vector PAV [147], it uses DHT instead of the routing table, to identify the different nodes, it employs a consistent hashing algorithm of IP NID, the PAV routing table PRT is the same like DSRP routing table and the difference is in the IP address of the next hop node is replaced with the successor NID, for that purpose, it relies on three algorithms:

- PAV route, discovering algorithm,

- PRT querying algorithm: to manage querying messages,

- PAV route maintenance algorithm: to manage joining and depart of nodes.

This method is more efficient than traditional AODV, although it has a problem with Node Storage Overhead.

In [148], the proposed method uses enhanced Chord (i.e., Mobile Chord) to resolve P2P problems over VANET like request messages managing, join , and departure of nodes. MChord is based on Chord with four modifications:

1. Aggressive table update: Nodes have to update all possible fingers, because usually in the sparse networks, two or more fingers can index the same node,

2. Overlay table broadcasting: Every node has a knowledge table with the other nodes information, and in every moment, every node must exchange updated information with one hop neighbors, all that to avoid Ping messages for DHT update, using a lot of routing messages and Packets lose, 


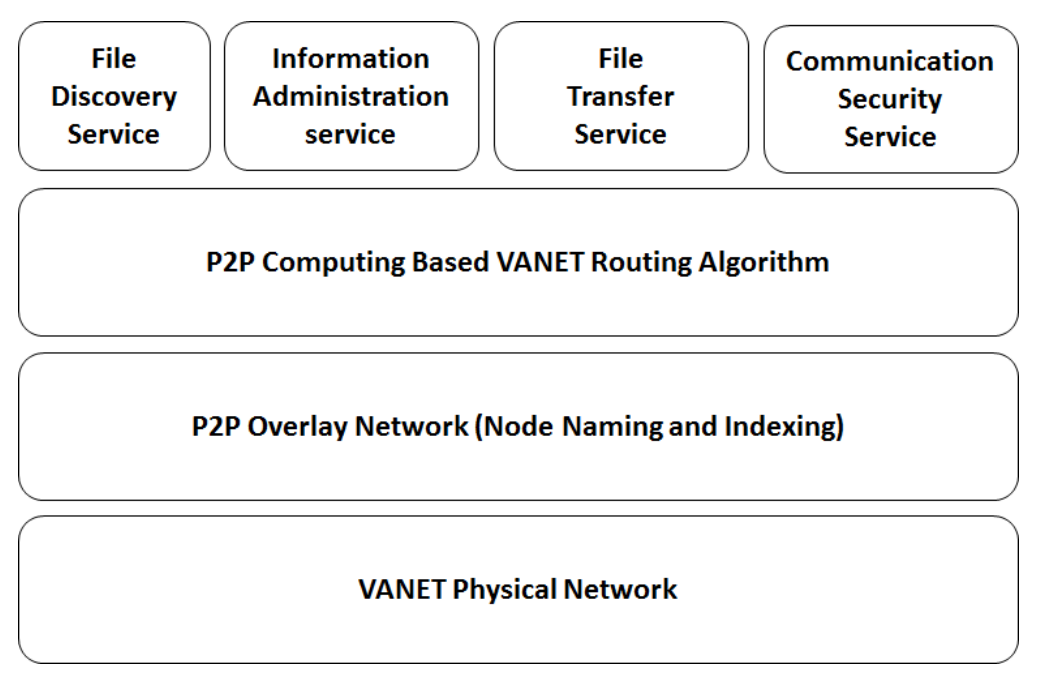

Figure 14: PAV Architecture [147]

3. Greedy forwarding: Every node sends application packet to the node who got the closest ID to Destination ID (using Knowledge table),

4. Passive bootstrapping: If a node wants to join the overlay, it must take a moment exchange knowledge table with his one hop neighbors without using hook node.

According to the simulation, the bootstrapping technique is more efficient than utilizing a hook node to join the overlay, and MChord outperforms Chord in terms of overlay consistency. Average request delay, correct response number, and response delay.

PeerTIS [149] is a traffic information system based on cellular network (i.e., mobile internet) to share traffic situation, it uses a modified CAN overlay for P2P sharing and GPS for route guidance. This technique uses GPS coordinates like a key (i.e., 2-dimensional keys) instead of IP address (i.e., for peers and route segments), the key space is divided into lots of zones, and each peer is responsible for the information of one zone. If a new peer wants to join the overlay, it must cooperate with the Bootstrap DNS server and it must divide a zone with another peer and update the road information. If a peer wants to leave, its zone must be merged with another one who belongs to another node. The peers are connected in the overlay if they share the same border and every peer has the address of their neighbors in the overlay. If a peer wants the information about a segment of the road, it must request the peer responsible, and if this peer goes to this road, it must update the segments information. In Vanet, PeerTIS is more efficient than CAN because it improves the lookup and updates messages. In addition, it presents a new dynamic navigation system to assist the driver in selecting the most appropriate route. However, this solution requires using a cellular network to access the internet, GPS errors might affect system performance, and it relies on the Bootstrap DNS server for node joining, which is unreliable (ID problems if many vehicles are in the same place).

N. Dutta [150] design a new DHT based p2p technique, this technique divides the map into many zones and each zone has a zip code, each zone and each file is hashed in a ring 


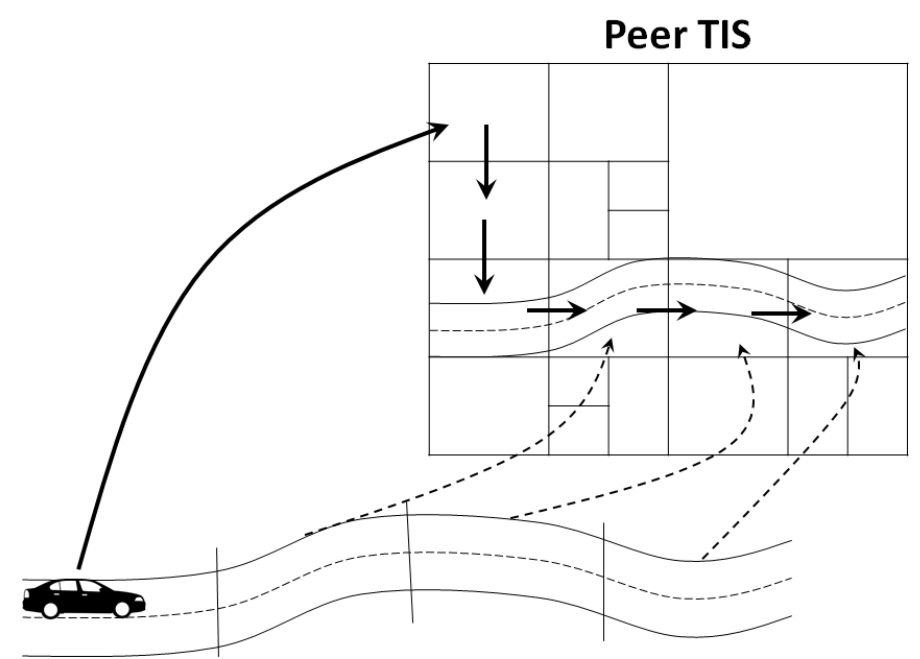

Figure 15: A P2P Traffic Information System [149]

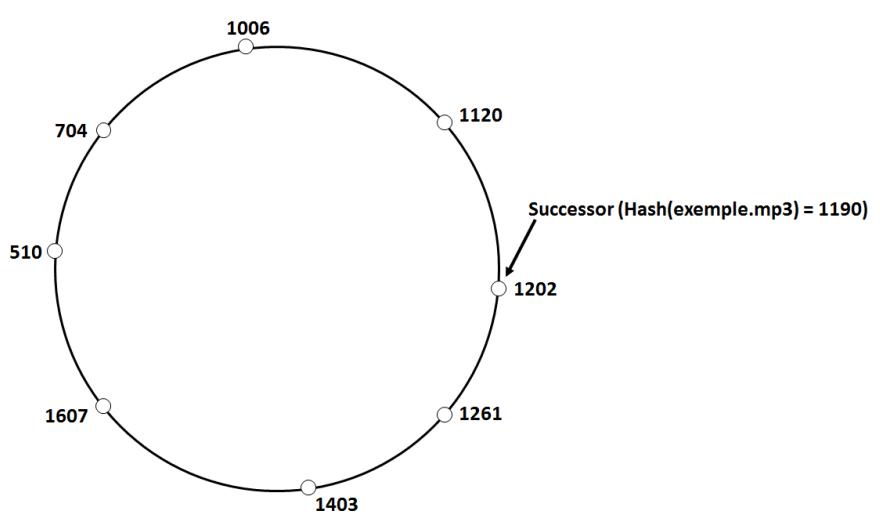

Figure 16: Chord Ring [150]

(i.e., SHA-1). Each file is assigned by the successor zone in the ring, so If a car wants a file, it will hash the name of a file and ask the cars of the successor zone for the file. This strategy is scalable and distributed, and it can be effective in a network with fewer users. However, the vehicles can switch the zone and possess the files; the vehicles must have an ID to reduce request time (request a vehicle, not a zone); and they must at the very least test this technique with simulation.

Another study [151] designs a cross-layer to improve Mobile Chord (CL MChord) [148] in order to enhance the information sharing techniques over VANET (see., Figure 17).

There are two modifications:

1. Cross-layer knowledge harvesting: The idea is to exploit the nature of wireless nature, every neighbor node can listen to other transmission packets even if they do not concern itself, and The MAC layer must send the important information to the application layer, so it can update the overlay table, 


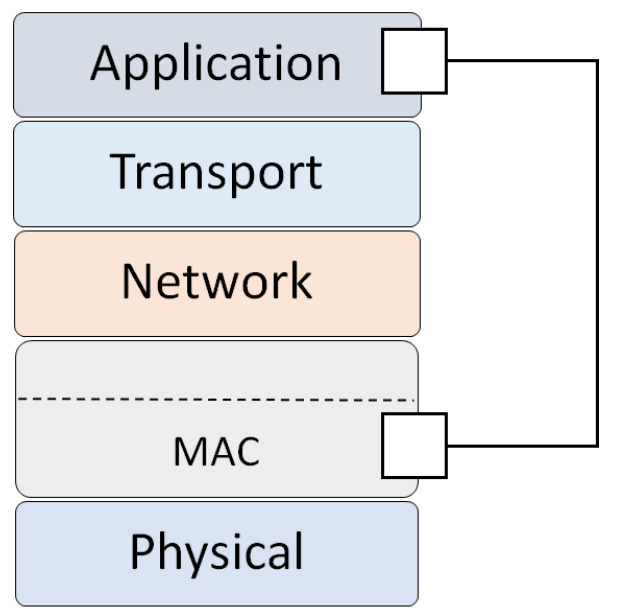

Figure 17: Cross-layer design [151]

2. Knowledge piggyback in overlay signalling: This proposed mechanism piggyback the overlay table in the Chord maintenance signalling packets and query packets, if a node receives a packet, it must extract the overlay table, updates his own table, and forwards the updated overlay table, so the neighbors nodes can have the updated overlay table (i.e., using Cross-layer knowledge harvesting).

This solution allows the overlay table to be broadcast without affecting the network. As a result, passive Bootstrapping is more efficient than Hook Nodes and avoids congestion around the Hook Nodes. This Mobile Chord improvement outperforms Mobile Chord and Chord in many aspects. However, it can also be compared to other peer-to-peer approaches such as CAN, MCAN, and Pastry. There are few changes between Mobile Chord and Cross-Layer Mobile Chord in the simulation, and the relationship between groups is still a concern. GraphTIS [152] is another traffic information system based on P2P overlay and cellular network, It is a modification of another old traffic information system PeerTIS [149] (e.g., CAN), the network is represented by a Graph (i.e., key neighbor graph), the vertices represent the road and the edges represent the intersections between roads, the graph is divided into a lot of sous graphs, each peer is responsible for one sub-graph. The roads between sous graphs represent the link between peers in the overlay. If a peer joins the overlay, it must be accountable for one subgraph. However, if it leaves, it must give back its sub-graph, so When a peer wants the information about an itinerary, it must send the request to the peer responsible for the first segment of this itinerary, and then it can process the shortest path to the destination (i.e., list of neighboring peers) using the key neighbor graph, to improve the lookup in the overlay, the authors use:

- An additional random link in the key neighbor graph, and it is not used in the shortest path procedure,

- Caching: to exploit the temporal correlations, so each peer must save the information about the peers responsible for some roads using requests and the update messages. 
This strategy improves the cooperative information system lockup performance (compared with CAN and PeerTIS). It represents the map using a key neighbor graph, which allows it to master the overlay information. It also utilizes a caching approach to avoid sending redundant messages. However, this strategy necessitates ample memory space for Caching, the map, the neighbor's key graph, the subgraph information, and the additional random linkages.

Another structured overlay technique was proposed named LAar-VANET [153]. It is a replica mechanism combined with a locality-aware approach over a structured P2P overlay in VANET. The proposition uses Chord DHT overlay with two mains modifications:

- Data replication in the owner node's finger node list addresses a data loss issue caused by node churn.

- The overlay is built using a locality awareness application that considers the physical position.

In terms of lookup success ratio, end-to-end time, and the number of logical lookup hops, simulations reveal that LAar-VANET outperforms Mobile Chord. However, if the number of buses representing the Bootstrap Vehicle (BV) is few, it will be fragile.

[154] discusses another interesting P2P system in the VANET. It uses the physical group clustering approach, and the pyramid tree algorithm is used for inter-cluster communication. It has several advantages, including data query and delivery efficiency, location independence, and rapid deployment in the real world.

\subsubsection{Two-Tiers Overlay Techniques}

In [155], the authors offer a new design of publish/subscribe architecture based on Chord overlay for VANET. The buses (i.e., Brokers) constructs the Chord ring Overlay (i.e., connected via mobile internet), and the vehicle can publish and subscribe. Each bus is responsible for a predefined number of vehicles (Hash (BusIP) is the successor to Hash(VehicleIP)), and every vehicle has a:

- Subscription Table and Publication Table: to save the active local sub/pub,

- Forwarding Table: to save the others vehicles sub/pub,

- Last Mobile Brokers: to save the ID of the last contacted Bus.

This protocol is divided into 5 procedures:

1. DHT formation of mobile brokers,

2. Publication/Subscription routing and storage,

3. Locating the subscriber vehicle,

4. Notification routing and delivery, 

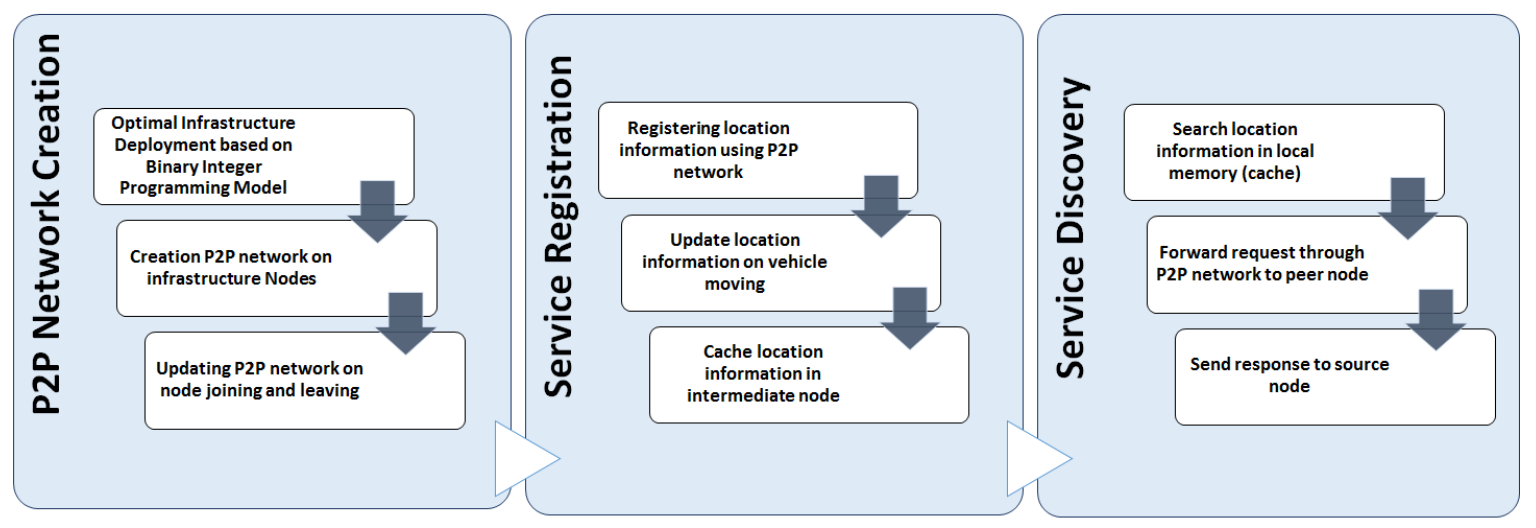

Figure 18: A service discovery framework installed in VANETs with infrastructure [156]

\section{Opportunistic delivery of notification.}

This method outperforms both the stationary infrastructure-based and the pure Vanet approaches. This method is simple to implement (Low cost). The lookup is improved by using Chord overlay. However, Chord overlay must be maintained over the internet. In addition, if a bus disconnects, all of its data will be lost.

The latest solution is described in [156]. It is a design of a service discovery framework installed in VANETs with infrastructure, this framework aims to discover services (i.e., vehicles information) with the help of structures P2P overlay (i.e., Chord) and it is divided into three steps:

1. Creation of P2P network: The RSUs are placed in the city using the Binary Integer Programming model, according to zones density, connectivity of the network, and the cost of the infrastructure. To manage the network, they use the DHT and a Chord overlay (i.e., each RSU is responsible for several vehicles),

2. Service registration: All information concerning a vehicle are saved in the responsible RSU $\left(R S U_{\text {Resp }}=\operatorname{succ}\left(h a s h\left(V_{I D}\right)\right)\right.$, every vehicle sends periodically (i.e., location, speed, direction) to the overlay via the nearest RSU and the intermediate RSU can save the vehicle information momentarily,

3. Service Discovery: If a node wants the information about a vehicle, it sends a request to the cluster head (i.e., clustering is used), and the cluster head forwards it to the overlay via intermediate RSU. Finally, The responsible RSU (or a node in the path can respond) responds to this request.

In terms of query success rate, location finding delay, and infrastructure cost, this technique outperforms EMBLS and HMLS [157] (other service discovery methods). However, it is ineffective in metropolitan areas.

A new two-tier P2P overlay-based technique was proposed in [158]. It is based on clustering (Trust Group), and each group performs a Chord overlay between nodes. In addition, 


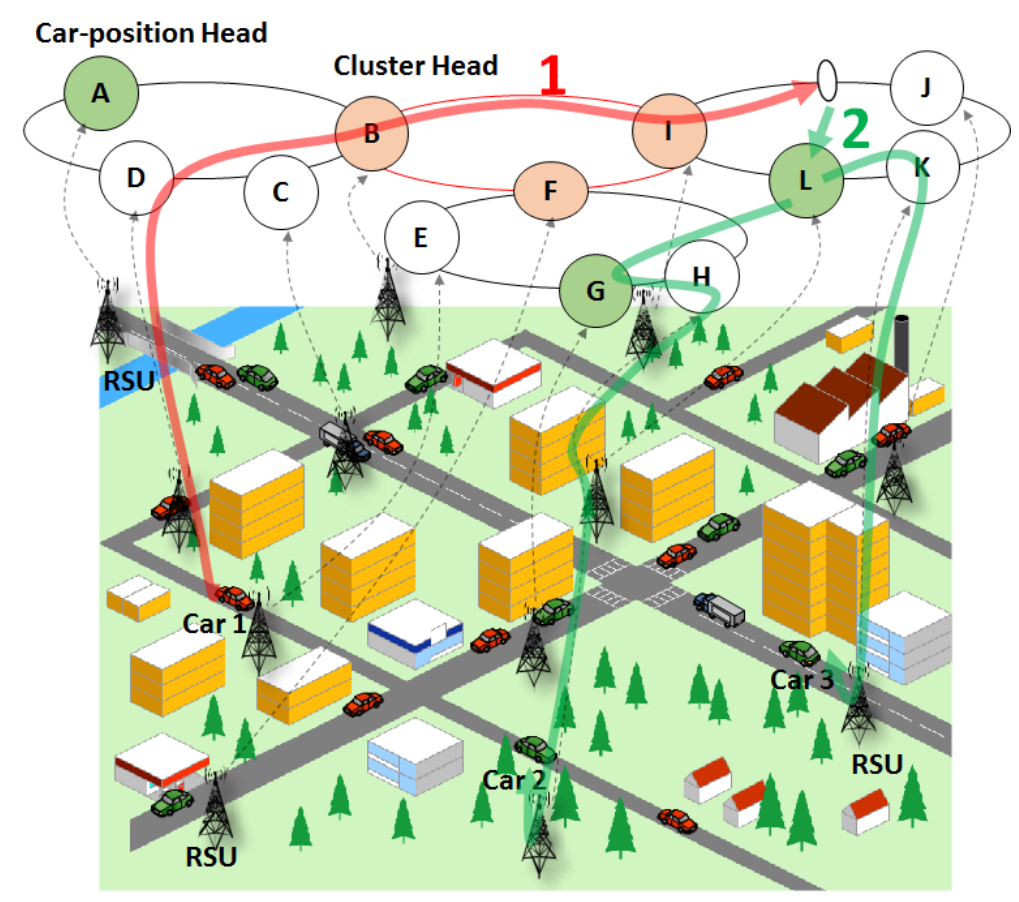

Figure 19: Urban Multi-Layered Chord (UML-Chord) [159]

each group selects a sort of cluster head to share the inter-cluster data with the global Chord overlay. This global P2P network connects the RSUs all over the city. This technique uses Machine Learning (ML) method named evidence theory to choose the best node from all the nodes in the groups to connect with the global overlay, depending on the reliability, the accuracy, the gradient information, and the learning parameters. This notion is more robust and less complex, but it comes at a higher cost in terms of computing and communication.

\subsubsection{Multi-Layered Overlay Techniques}

Urban Multi-Layered Chord (UML-Chord) is described by [159]. It uses the multi-layered Chord P2P file sharing, the network is divided into zones, each zone has an RSU, and this RSUs form a cluster, each cluster constructs an overlay (i.e., Chord ring), it has a cluster head and car-position head (cp-head). The cluster heads form another Chord ring (named Super ring), and the cp-head stores all the information about the vehicles in the cluster. The download process is based on two formulas to compute the connection lifetime and bandwidth. In several parameters, such as the number of hops, control message overhead, and query latency time, this strategy outperforms Chord and Overlay-based solutions. However, a large number of chord rings can result in an overhead. Therefore, it has been compared to older approaches like Chord.

In the end, the following diagram presents a taxonomic of structured overlays for VANET (see., Figure 20). 


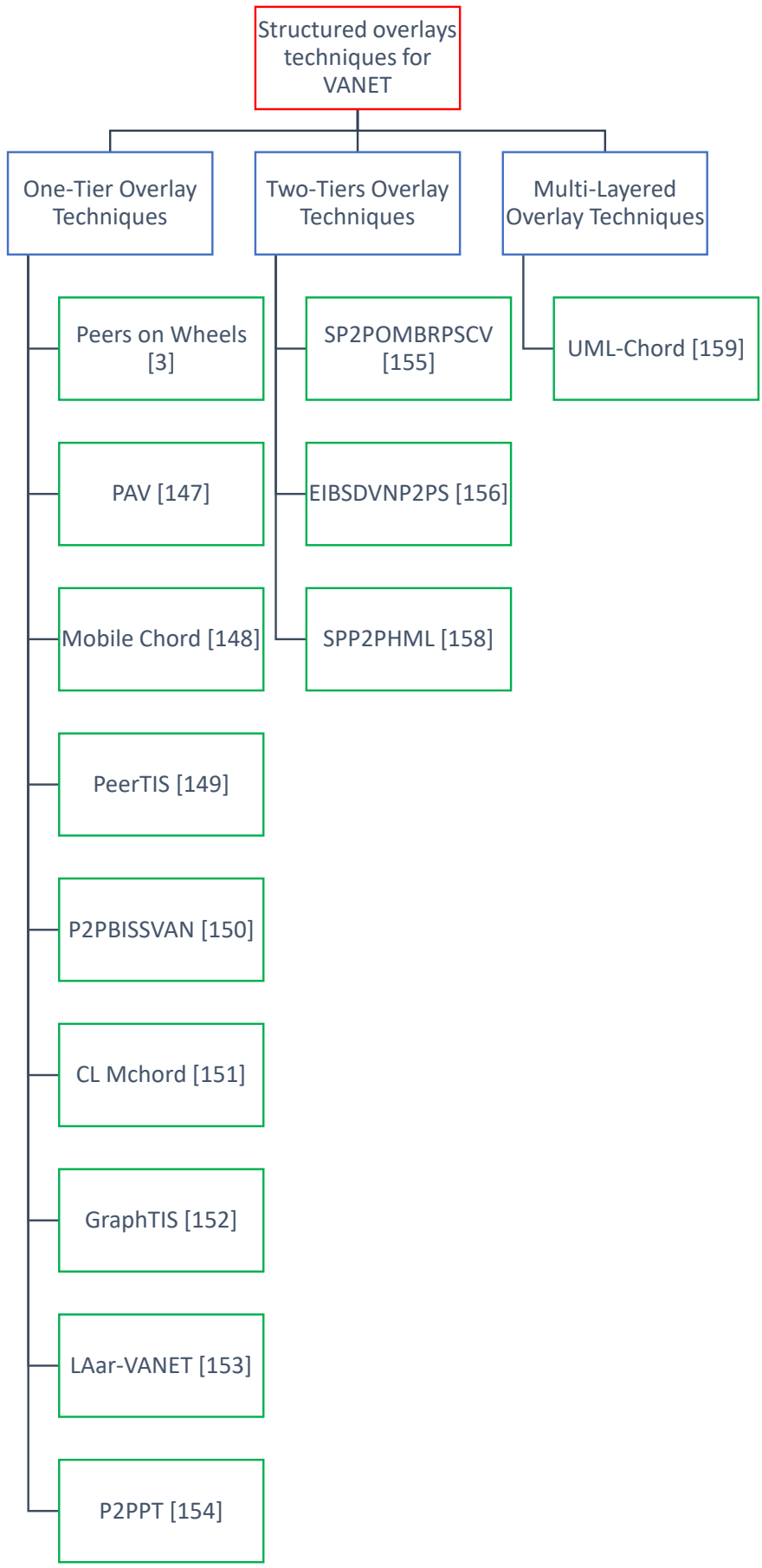

Figure 20: Taxonomic of structured overlays techniques for VANET. 


\subsection{Unstructured overlays techniques for VANET}

Unlike the structured overlays, the unstructured overlays ensure the flexibility of nodes. So this type of overlay can facilitate node membership, reduce the maintainability cost, and decrease messages overhead, but the major problem is the lookup process. In the following, we will discuss and study the most interesting unstructured $\mathrm{P} 2 \mathrm{P}$ overlays in a detailed manner.

\subsubsection{One-Tier Overlay Techniques}

Another solution that uses safety messages is in [160]. This P2P technique is dedicated to neighboring vehicles in the same direction. The idea is to divide the network into a lot of Local Peers Groups (LPG) with two communication types: Intra LPG and Inter LPG. For that purpose, it proposes two different clustering architectures (i.e., Dynamic and Stationary LPG). In Dynamic Clustering, the roadway is divided into lots of Zip-code areas that define the LPG depending on the communication range, number of vehicles we want to be in an LPG, road conditions, and LPG's members dynamically change as vehicles move. However, in Stationary Clustering, the vehicles in the neighborhood can construct an LPG with Relative Ordering (LPG-RO) and LPG with Linked Equivalent Cells (LPG-LEC). It can be utilized on city or highway routes, however if the network is crowded, it will have a high overhead.

The results offered by T. Atéchian et al. in CoFFee [161] suggest the use of the following ideas:

- Geocast Routing Protocol (DG-CastoR): It is a new routing protocol to route the request messages based on mobility prediction to estimate the communication time between the sender and the receiver nodes.

- Content Distribution management: Used to share files, It is dependent on neighbors exchanging periodic Hello messages to confirm their availability. Every file is separated into sections, much like a tree (i.e., every piece into many parts). The provider node uses the communication time prediction formula to send the rarest pieces first (i.e., divide and prune functions).

In terms of block transfer rate and network overload, this solution outperforms the random method. It forwards files using a prediction trajectory function. To distribute the rarest components, dividing files into a tree structure is a brilliant idea. However, sending hello messages on a regular basis and creating an overlay for each request overloads the network. The requester node's trajectory varies, resulting in an incorrect prediction. This methodology is compared to the random method rather than other methods.

H. Perkuhn et al. [162] developed an application that combines a GIS with a P2P system to share information. It uses mobile communication (i.e., 3G) as a default communication besides a VANET communication (i.e., P2P over VANET) in the case of the 3G network cannot provide the communication like: in the tunnels, the charge of the cellular antenna. This system detects the limit of the cellular network and establishes a P2P communication. For this purpose, the system offers a list of nodes with their IPs that can provide the resources 


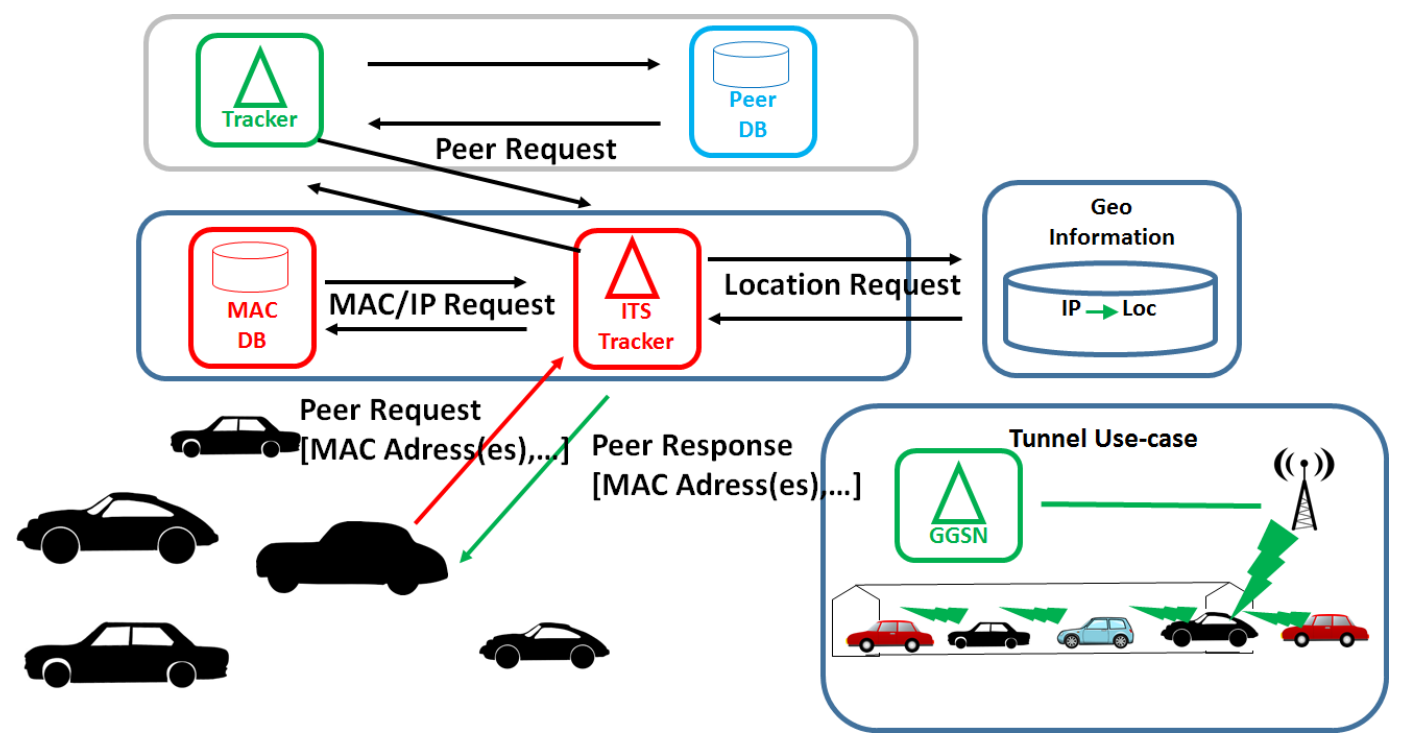

Figure 21: Schematic Overview of the Geo-Supported P2P VANET for ITS Applications [162]

to the requester peers, and the requester peers can communicate with the seed(i.e., provider) $\mathrm{P} 2 \mathrm{P}$ communication( using Bit torrent). It is a new hybrid application that can overcome the limitations of cellular networks by delivering content to many peers in a given area at the same time. However, because multiple peers can request the same peer at the same time, this may result in overhead. In addition, the response time can be huge, and the network can change constantly. Thus it must be compared to other systems, at the very least through simulation.

N. Ahmadifard et al [163] propose a new file sharing protocol in the VANET (ISEFF) (i.e., Without infrastructure) using Bit torrent and consistent hashing to Improve sharing performance. This protocol is divided into five mechanisms:

\section{Mapping}

\section{DHT establishing}

\section{Searching of the requested file and finding owners}

4. Piece and owner selection strategies and retrieving the requested file

\section{Updating DHT}

(a) If a vehicle changes the region, it must update the DHT from the first neighbor vehicle,

(b) If a vehicle receives the first piece of a file, it must send a NMsg to the mapped region.

In terms of received file ratio, average download time, average file download, and control message overhead, this technique outperforms the P2P-based approach. Only a few vehicles 
keep track of the files to avoid the network load. The advantages of using BitTorrent are to increase file reception rates and download times. However, the dynamic changing of the regions creates an aggressive Update, and timer optimization remains a concern.

In [164], A. Valibak et al. design a cooperative traffic information system using P2P file sharing and clustering in the urban zones. It is a two purpose system: The best path selection (i.e., for vehicles) and the best-connected path (i.e., for request messages). Each vehicle has a GPS, digital geographic map and 802.11p interface, and each node in the network has three tables:

1. Travel Time table: to save the travel time for each route segment,

2. Neighboring Table: to keep the information about the vehicles in the same segment,

3. Connectivity Probability Table: to save the connectivity probability for each segment.

Each peer must update its tables continuously by neighboring peer messages and by self computations. The peers are organized in clusters, the nearest peer to the intersection is the cluster head, and the cluster head is responsible for the communications between the local segment peers and the other segment peers. If a vehicle arrives at the intersection, it must calculate the best path to the destination using the updated information in the travel time table and DIJKSTRA algorithm, as it is illustrated in 22. If the peer does not have the information about a segment (or out of date), this peer must send a request packet to the peers in the requested segment with the best-connected path (i.e., Connectivity Probability Table):

$$
\begin{gathered}
P_{\text {Path }}=\left(\sum_{i=1}^{m} P_{i} L_{i}\right) /\left(\sum_{i=1}^{m} L_{i}\right) \\
P_{\text {Connectivity }}=\left(\sum_{i=1}^{n+2} d_{i}\right) / L \quad d_{i}= \begin{cases}d & , d<R \\
R & , d \geq R\end{cases}
\end{gathered}
$$

Where $m$ is the number of segments in the path, $d$ is the distance between two peers, and $R$ is the communication range. This strategy can decrease travel time in urban areas, optimize control messages to avoid bandwidth waste, and guarantee link connectivity, all of which increase response time. However, in the simulation, they utilize a limited comparison criterion, and the threshold $\mathrm{T}$ (of update time) must be optimized.

The objective of M. Picone et al. in their book [165] is to propose a new unstructured P2P overlay for an ITS that uses the Geographic position to form the clusters, decentralized infrastructure-based system, and a P2P overlay scheme named Distributed Geographical Table (DGT). The purpose of this design is to make information retrieval more efficient. The network data is saved in a distributed manner by the DGT, the overlay's neighboring peers are geographically adjacent, and clustering methods are not used to prevent peers' saturation. However, the use of periodic messages continues to be a concern. It is not possible to use it in a sparse network. It also has to be compared to other systems. 


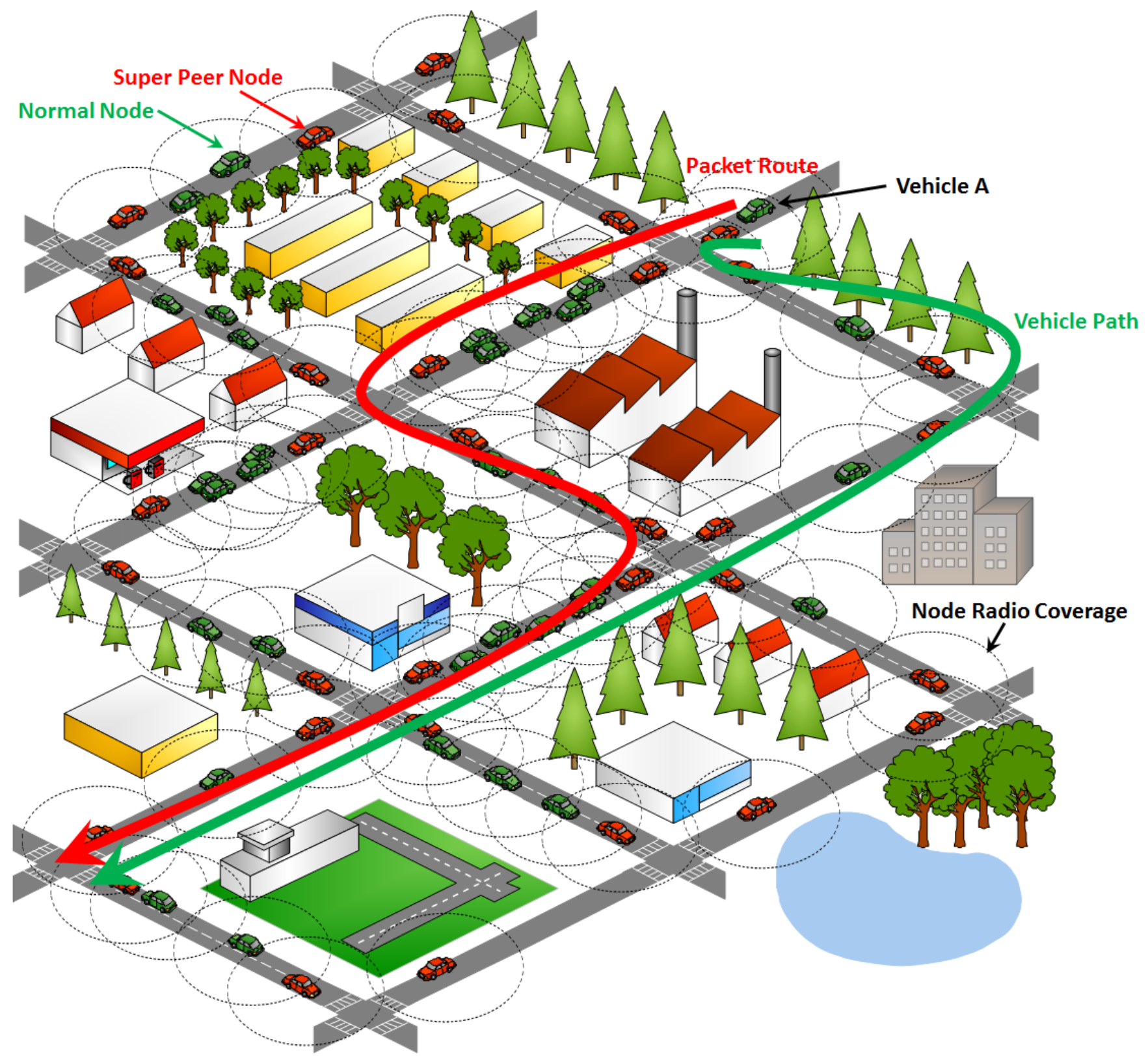

Figure 22: A cooperative traffic information system using P2P file sharing and clustering in the urban zones [164] 
In this study, a new technique that improves the message dissemination technique in VANETs applying P2P communications is suggested [166]. This technique uses the link sustainability between peers to select the cluster head and discover the roads, it is based on broadcasting for message dissemination, and it is divided into two parts: Clustering and Route Discovering, This approach is more efficient than ASAP [134] regarding the number of exchanged packets, delivery time, and package transfer rate per time unit. It can anticipate the link lifetime in order to avoid disconnection. Furthermore, it can also assist in selecting the ideal cluster head (to avoid repeated lection). However, the cluster head must be involved in route discovery, and the network can be loaded by broadcasting and periodic messages.

A.A. Valibak el al [167] present a cooperative ITS in VANETs with the help of P2P communication (to improve [163]). This system has two purposes: finding the shortest path (i.e., time) for vehicles and routing the exchanged messages through the best-connected path simultaneously. To do that, it uses a clustering technique with a specific data structure (i.e., each node has specific tables) and different types of messages. The vehicles on the road are organized in clusters (i.e., depending on the road segment), the cluster head is the closest peer to the intersection. Each node in this system has three tables:

1. Travel Time table: for each segment, update data with aggregation $T_{\text {Agg }}=\alpha T_{\text {Old }}+$ $\beta T_{\text {New }}$

2. neighboring Table: updated periodically and in case of segment change, contain information about neighbors,

3. VANET Connectivity Table: updated in messages reception and periodically, include the radio connectivity probability of each segment.

There are four kinds of exchanged packets:

1. Normal Packets: to periodically (i.e., adaptive) exchange data between peers,

2. Super Peer packets: to exchange information between Super peers in each intersection,

3. Lookup Packets: to request the travel Time Information

4. Reply Packets: to reply to the lookup packets.

The radio connectivity probability of each segment (i.e., VANET Connectivity Table) is computed as follows:

$$
P_{\text {Connectivity }}=\left(\sum_{i=1}^{n+2} l_{i}\right) / L
$$

$L$ the road segment length and $l_{i}$ are the length between vehicles. This system can ensure that information about the route segment is available at the same time as the optimum path to the target is being retrieved. Furthermore, because this approach is dynamic, the best path can be adjusted according to road conditions. However, this technique cannot 
be employed on the highway (disconnection on lengthy highways), and several parameters, such as update time and thresholds, must be optimized.

The PEer-to-Peer protocol for Allocated REsource (PrEPARE) [168] is based on VANET. It supports information sharing without the use of RSUs or any infrastructure (based on the Gnutella protocol) Vehicles will need to collaborate in order to exchange and search for accessible information. The simulation results demonstrate that the proposed strategy has an impressive search time for inquiring sources in a single hop and searching for information in several hops. However, the method produces a high success rate with minimal overhead, resulting in significant resource availability. The researchers hope to develop a P2P protocol that makes resources available to vehicles and expands the amount of resources used in a vehicle cloud.

Another interesting technique is described in [169]. It's an Open p2p vehicular network library for real-world experiments. It exploits the libp2p (Goland library for P2P overlay) protocol library to construct a network and expose its vehicle trip parameter. It uses the gossip protocol and has demonstrated library functionality on an Android application in realworld conditions. It also uses 802.11ac protocol (as opposed to $802.11 \mathrm{p}$, a specially designed protocol for vehicular networks) Wi-Fi hardware, which is found in almost all smartphones today. Researchers will have autonomy in collecting appropriate trip parameters for their purposes and inferring study outcomes, but this is still not a VANET application, and simulation will be required to compare this application to others.

\subsubsection{Two-Tiers Overlay Techniques}

In [170], The authors proposed a hybrid framework (P2P and Internet) to share information over VANET using mobile and fixed nodes clustering. The Social Cluster-Based P2P Framework (SCBP2PF) is composed of:

1. Social Cluster-Based Overlay Structure: Mobile and fixed peers are grouped into (many) clusters using the following cluster mechanisms:

(a) Interest-Based Cluster Construction: Every cluster head must be a fixed node, each mobile node has a cluster head (i.e., must be similar using similarity function), and each information in the cluster must be registered with the cluster head,

(b) Small World Based Inter cluster Overlay Construction: Each cluster head can calculate the similarity with the other cluster heads and use Random Walk to be connected. After constructing the overlay, every node can use it to discover resources by sending a request message to the cluster head. If the cluster head has the information, it must transfer the data directly. If not, it must forward the request message to other cluster heads.

2. Life time-aware Flooding Scheme: It is used to control the broadcast of request messages by computing Link Expiration Time LET, LET is calculated with the position, direction, and speed. The node who got the long LET can forward the messages, 
3. Connectivity-aware Retrieval Scheme: Used to resolve a lot of problems like cutting paths and network load,.... It calculates the Path Life Time and Bandwidth with the help of respond packets header.

This solution outperforms older strategies in communication overhead, retrieval file ratio, and query hit ratio. However, even if the network uses an internet connection and RSUs, the periodic messages load the network.

In the aim to enhance the lookup success rate and avoid the disconnection problems of the VANET, S. -L. Tsao et al. [171] prefer to use VANET and unstructured p2p overlay to offer a better traffic information system. The neighboring peers forms a cluster and select their super peer depending on their IDs, and the super peer must not be further than $d$-hop to other peers. The peers in the cluster communicate with Inter vehicular communication IVC (i.e., lower tier), and the super peers are connected to construct an unstructured P2P overlay (i.e., High tier) like it is described in Fig 23. In terms of network connection, traffic information propagation, and lockup success rate, this strategy outperforms broadcasting, structured P2P overlay, and infrastructure-based P2P. On the other hand, the super-peers selection procedure needs to be investigated and is exclusively employed in traffic information systems. This solution outperforms older strategies in communication overhead, retrieval file ratio, and query hit ratio. However, even if the network uses an internet connection and RSUs, the periodic messages load the network.

J. Guo [172] choose to improve the link lifetime and resource lookup by enhancing the two-tier p2p communication system [171]. For this purpose, their technique has three modifications:

1. Reliable super-peer selection: The peer with the maximum predicted link lifetime between peers is selected to be the super peer, and the link lifetime between two peers is predicted as (see., Figure 24):

$$
\text { Tlink }_{i j}= \begin{cases}\frac{\sqrt{R^{2}-h^{2}}+\sqrt{d_{i j}^{2}-h^{2}}}{\left|v_{i}-v_{j}\right|} & \text {, When moving closer } \\ \frac{\sqrt{R^{2}-h^{2}}-\sqrt{d_{i j}^{2}-h^{2}}}{\left|v_{i}-v_{j}\right|} & \text {, When moving away }\end{cases}
$$

2. Pre-broadcast detection: to select between one-hop neighbors communication or high-tier request.

3. Location awareness access: physically neighbors nodes they can quickly exchange information.

This technique can help to solve link instability and resource discovering problems. Moreover, it is more efficient than the old version of two tiers p2p communication system. Nevertheless, the forming mechanism of the cluster is a big problem to resolve.

The idea of J.S.Li et al. [173] is to design some information localization management system (i.e., localization of vehicles in urban areas) based on VANET, P2P overlay, and a backbone network (i.e., wired). The map is divided into many equal zones. Each zone is 


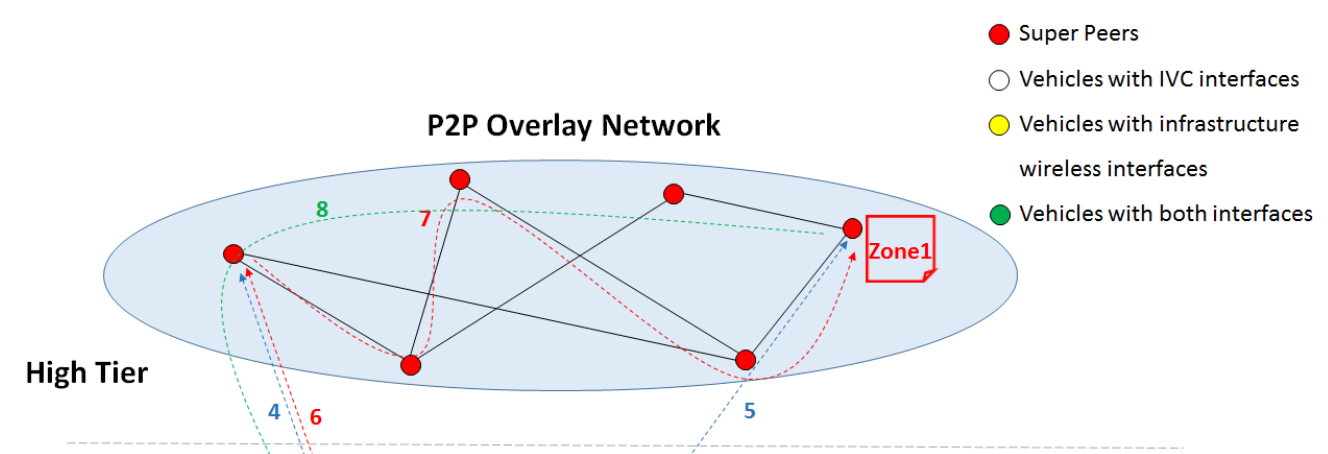

Low Tier

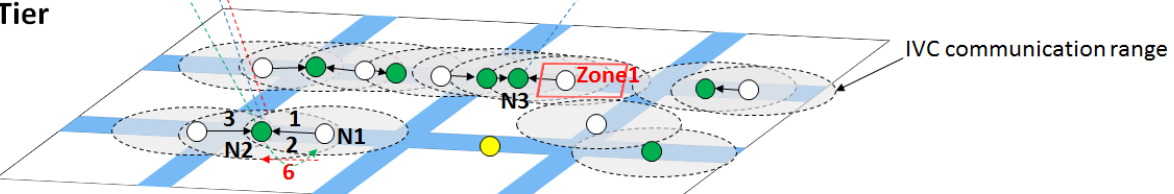

1- $\boldsymbol{N} 1$ joins the system via IVC

Vehicular Ad Hoc Network

2- $\mathbf{N 1}$ periodically disseminates traffic information

3- Vehicles elect super peers

4- $\boldsymbol{N} \mathbf{2}$ becomes a super peer and joins the P2P overlay

5- N3 maintains the traffic reports received from neighbouring nodes within a cluster

6- $\mathbf{N 1}$ queries traffic information of Zone1 via IVC

7- $\boldsymbol{N} 2$ forwards the query through the P2P overlay using geographic routing

8- N3 responds the traffic report of Zone1 to $\mathbf{N} 1$ through $\mathbf{N 2}$

Figure 23: Design of a two-tier P2P traffic information system [171]

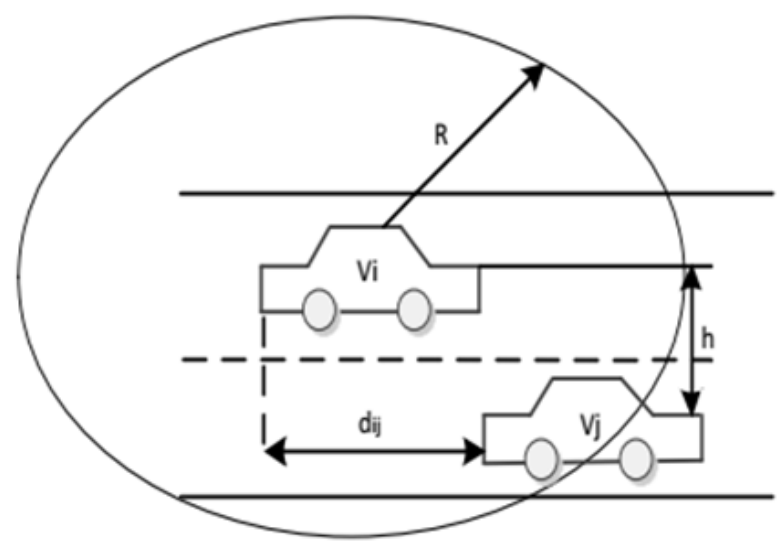

Figure 24: Predicted link lifetime between two peers [172] 
covered by one RSU (i.e., Location Information Point LIP). Each LIP has the information about the vehicles in its coverage zone (in the vehicle table). The LIPs with a significant number of vehicles (i.e., more than a threshold) are named Hotspot LIPs (i.e., this Hotspot LIPs represents the peers in the P2P overlay (i.e., flooding based)), so If a vehicle Vs want to find another $V d$, It must follow the algorithm detailed in [173]. The usage of Hotspot LIPs increases the success rate of vehicle lockup. As a result, this technique has a faster response time and a higher lockup success rate than RLSMP. However, the overlay is used in one wired network (Hotspot LIPs), and it uses a flooding-based overlay. It is dedicated only to vehicle localization.

$\mathrm{K}$. Lu et al. [174] developed an adaptive routing protocol used in the traffic information systems. Its goal is to improve the lookup efficiency of traffic information in the two-tier traffic information system proposed by S. L. Tsao et al.[171]. The two-tier traffic information system uses P2P overlay and VANET for the lookup of information, and the proposed algorithm helps the system to choose the best way for the lookup of traffic information (i.e., VANET or P2P overlay). That decision depends on the estimation of routing success probability. This algorithm has improved the lookup success rate of the two-tier system. In addition, it aids in determining which approach (Vanet or P2P overlay) should be utilized to find the shortest path. However, the time it takes to compute the probability causes a delay in response to the request. Another issue to overcome is optimizing the value of the threshold P.

In [175], it was shown that P2P overlay through infrastructure-based communication (i.e., higher tier) and VANET IVC (i.e., lower tier) could improve the information retrieval systems, so the vehicles in the network are organized in many clusters, and each cluster has a Super peer, each node in the cluster has an $\boldsymbol{I D}$, and the nodes with the lowest ID is the Super peer, this super-peer represents a bridge between the two-tier (i.e., from the nodes in the cluster to the highest tier), due to the high mobility of vehicles the super-peer and the periodic changing topology of $\mathrm{P} 2 \mathrm{P}$ overlay, the nodes in the same cluster must share all the available information,

- If a user in the vehicle wants some information about traffic or another service, a request must be sent in VANETs (i.e., hop by hop with $\boldsymbol{T} \boldsymbol{T L}$ mechanism and geographic forwarding scheme),

- If there is no answer, the super-peer of this cluster uses the Gnutella-based P2P overlay and geographic routing mechanism for the lookup of this information,

- The super-peer sends a request with TTL in the overlay (i.e., to his neighbor). If a node in the overlay receives the request and the requested information is not stored in its cluster, it must forward the request to his neighbor until the request is responded or $T T L=0$.

Compared to Broadcasting, Structured P2P overlay, and infrastructure-based P2P, this method is superior in terms of network connection, traffic information dissemination, and lockup success rate. 


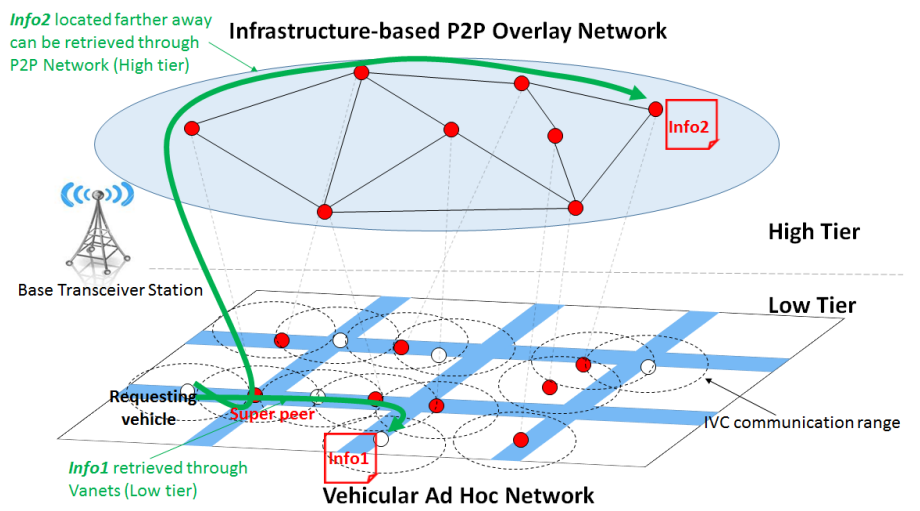

Figure 25: P2P overlay through infrastructure based communication and VANET IVC [175]

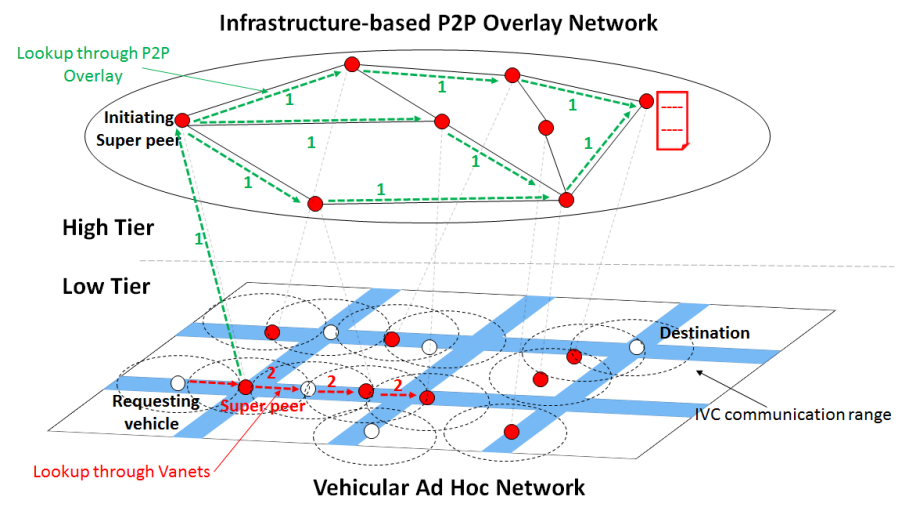

Figure 26: Adaptive Lookup Protocol for Two-Tier VANET/P2P Information Retrieval Services [176]

Like most authors, C. Cheng et al. [176] design a two-tier (i.e., P2P/VANET) architecture for VANET based on clustering. The conventional VANET is used for intra-cluster communication, and P2P overlay over an infrastructure-based network is used between Super Peers (i.e., cluster head). It is a modification of an older two-tier architecture [171], the difference is the use of reachability information, Bloom filter, and probabilistic information to choose the best lookup way: P2P overlay (unstructured/ Gnutella ) or VANET. This concept is illustrated in the following diagram (see., Figure 26). In terms of lookup success rate, lookup overhead, and lookup latency, this technique outperforms the conventional Two Tiers technique. However, optimization of the thresholds (threshold H,threshold L,P) is an outstanding topic. If this technique is employed in ITS, the peer must submit a request for each road segment, which might result in broadcast storms. Additionally, the usage of unstructured overlay increases response time.

Based on the approach presented in [177], they explore the possibility of using P2P over VANET with the help of autonomous clustering management of the nodes. For that purpose, the authors use VANET communication in the cluster and Cellular (i.e., 3G /LTE) communication outside the cluster. This clustering technique is more effective at disseminat- 


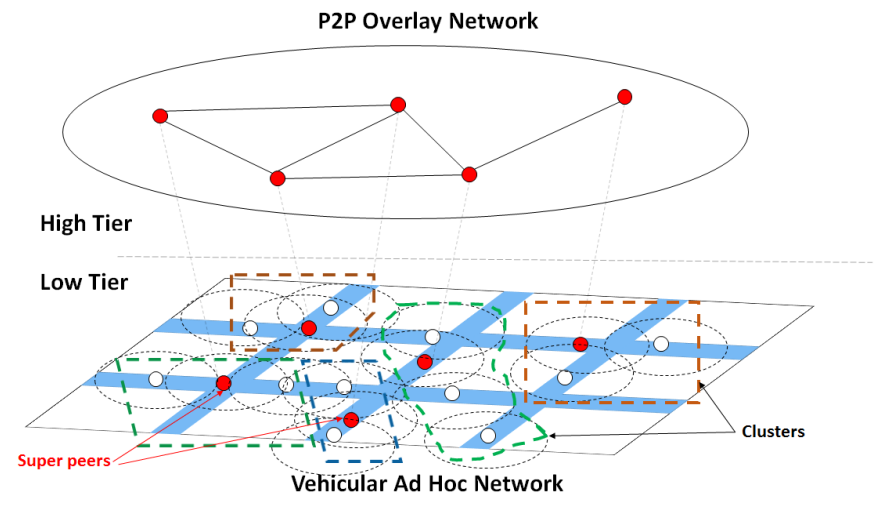

Figure 27: Autonomous clustering management of the nodes [177]

ing information than basic Vanet. The cluster heads are dynamically selected based on the network topology, facilitating communication. However, it makes use of periodic messages between surrounding nodes, which might load the network. In addition, 5G technologies can improve cellular communications.

As the precedent proposition, an adaptive lookup two-tier approach [178] employs the idea of reachability for information retrieval. It is founded on two properties:

- Because the RSUs function as a super-peer, no super-peer election is required, reducing the protocol's complexity.

- According to the authors, the structured overlay is not ideal for vehicle environments; thus, in this P2P system, an unstructured overlay network is employed.

- This study employs the distance-based reachability model to reduce lookup latency while retaining a reasonable lookup success rate.

When comparing this Adaptive lookup two-tier mechanism (ALTM) to the Conventional two-tier lookup mechanism (CON) with NS-2, this strategy reduces the search success rate, data flow might be prioritized to improve it. Prioritized data refers to the modification of data contention duration according to its relevance. However, the idea of a dynamic distance threshold is ignored. Instead, it indicates that the distance threshold should differ depending on the vehicle density.

\subsubsection{Multi-Layered Overlay Techniques}

A geographic P2P system to discover and share resources over VANET is developed by Y. H. Lee et al. [179]. This system is based on a backbone architecture to restrict the massage exchange zone to avoid network load and clustering mechanism. The network is divided into grids, every grid has a grid header, and the grid header is responsible for the grid information. A coordinator is a grid header used to coordinate with the grid headers. Each grid header has a Multi-piece Item table (MPI) with information about the pieces in the grid (piece ID, piece size, hash ID, location). Each coordinator has a Tracker List 
Table (TLT) with resource information (resource ID, resource name, resource size, hash ID). And the resource discovery and sharing algorithm is detailed in [179]. This strategy outperforms Gnuttela regarding satisfaction rate and downloads time, particularly when the Nearest first piece selection policy is used. The Grid header and Coordinator concepts can help reduce network load. This technique is capable of locating resources and optimizing the time required for dissemination. However, it can be compared to other approaches. Disabling the Grid header/coordinator cripples the system, and backbone system messages overload the network.

To conclude this section, here is a diagram which presents a taxonomic tree of independent superposition techniques for VANET(see., Figure 28).

\section{Comparative Study}

From the study made previously and the comparison between the different $\mathrm{P} 2 \mathrm{P}$ techniques in VANETs summarized in the following table (Table 1):

We can conclude that, first of all, the overlay independent techniques tried to improve P2P methods without modifying the overlay type or structure. But the real strength of $\mathrm{P} 2 \mathrm{P}$ algorithms is in the overlay because new techniques like clustering methods for network coding cannot resolve P2P network problems, and the most crucial issue in VANETs is the lookup process that is insured by the overlay.

The second remark concerning the structured overlay is that this overlay helps us very well in the lookup process and can reduce the network load, but the high mobility of nodes destabilizes the overlay. To solve this issue, several authors use two-tier overlay architecture. This kind of design involves the most important nodes in the overlay, not all of them.

Finally, unstructured overlays are adequate than structured ones in high mobility, but the lookup time is considerable. So many authors try to avoid this problem by including two-tiers or multilayered architectures. But other ones prefer to integrate the GPS position in the overlay structure (two purpose techniques), such as ITS.

\section{Open issues and future research directions}

Even though there is a wide study in VANETs on the requirements' efficiency, robustness, and scalability, a significant number of challenges are still to be addressed, such as the high mobility, connectivity, information lookup process, network load, and management of joining and departure of nodes. In this section, we discuss these challenges and present potential future directions detailed in table 2 with their proposed solutions, and recommended references.

\subsection{Unmanned aerial vehicle (UAV) based P2P communications}

Unmanned aerial vehicles (UAVs) equipped with sensors and wireless interfaces could collect and share a significant amount of data. This kind of system has to avoid collisions between UAVs and ensure cooperative synchronization[180]. Therefore, it could be an interesting solution if we deploy a set of UAVs hovering over VANETs and playing the role of relays to bridge communication gaps between vehicles. 


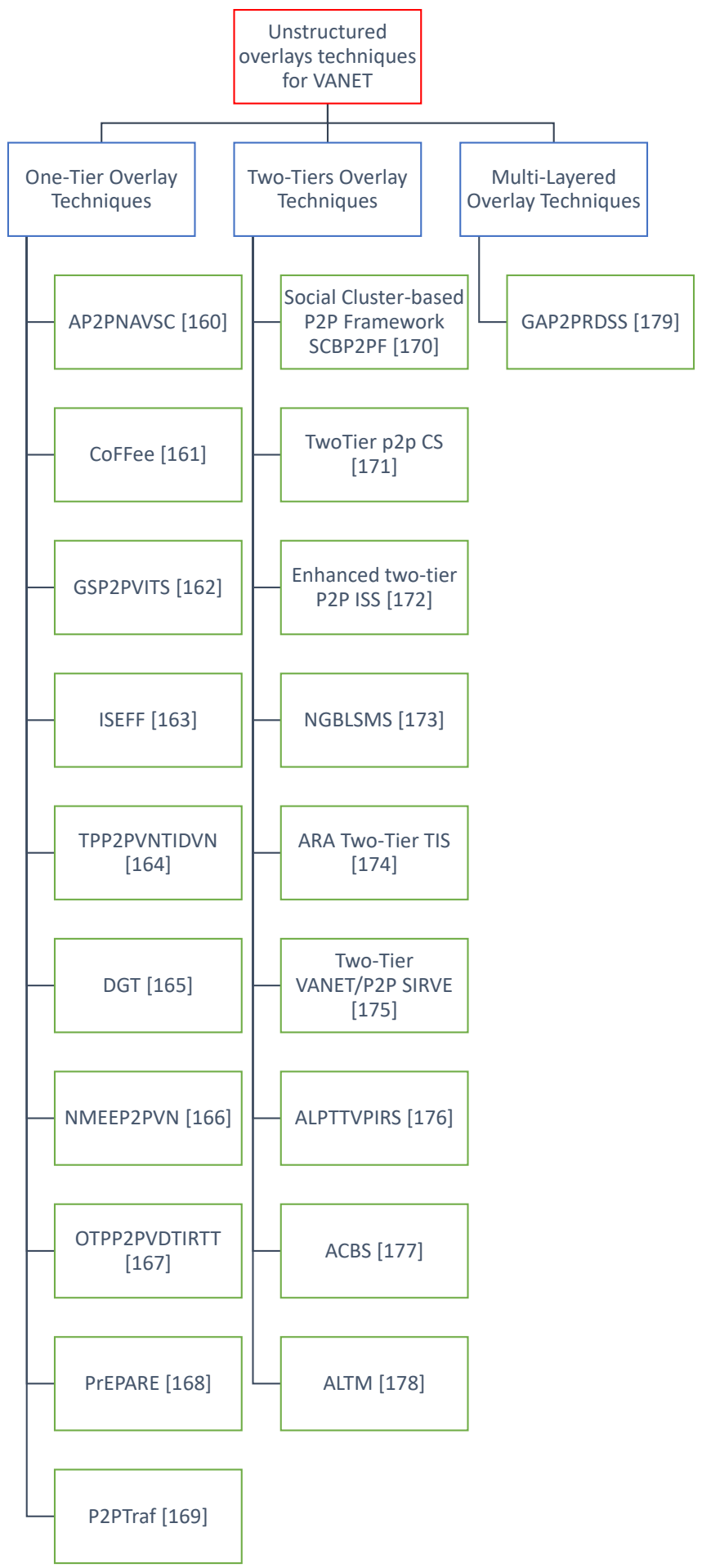

Figure 28: Taxonomic of unstructured overlays techniques for VANET. 
Table 1: Comparative study between VANET P2P Techniques.

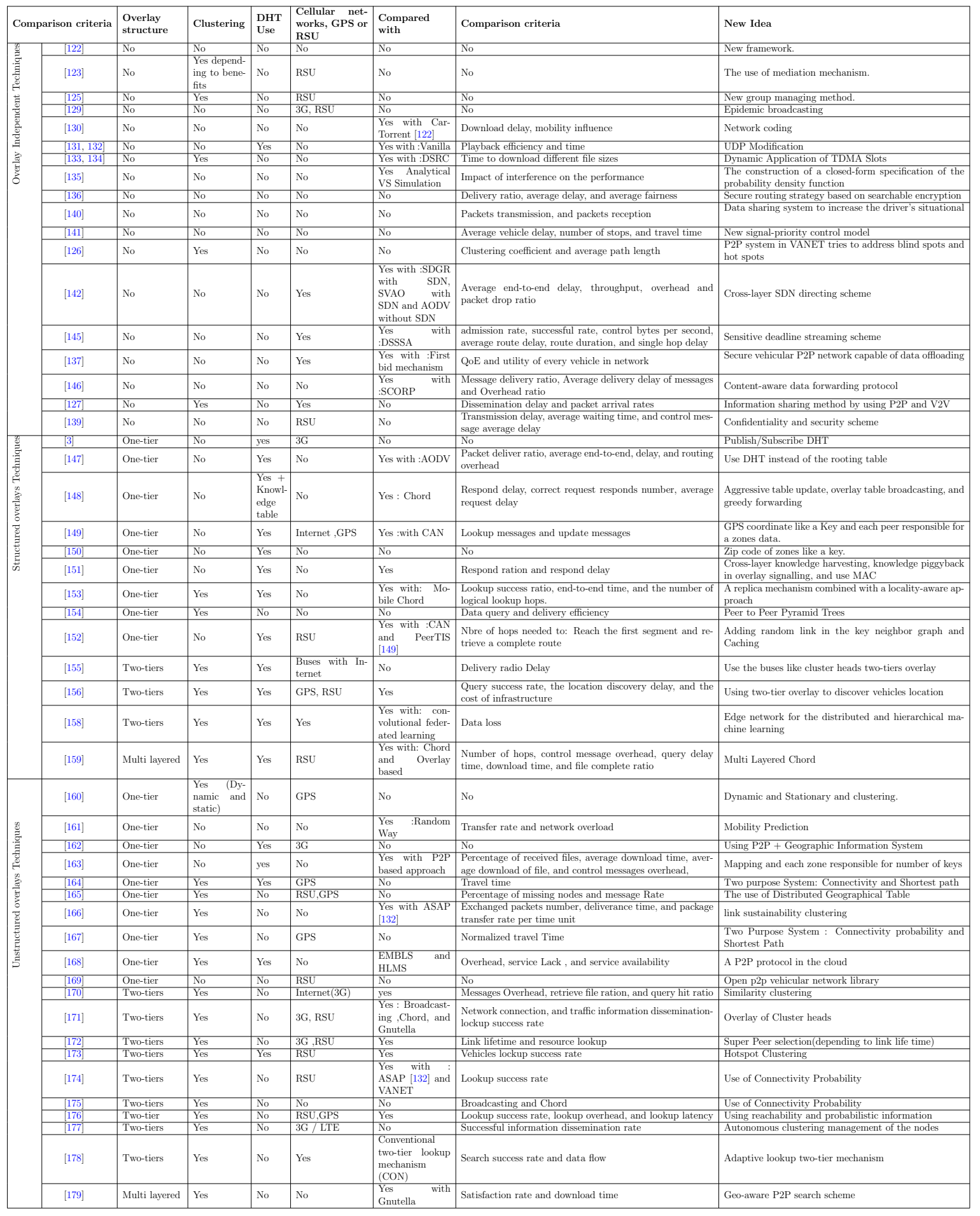




\subsection{Enhancing P2P overlays with machine learning techniques}

Machine learning (ML)[181] and deep learning (DL)[182] are powerful Artificial intelligence (AI) tools. Many authors tries to solve crucial problems in P2P by using these tools. In [181], they introduce a novel application of machine learning to the neighbor selection problem in self-reorganizing networks. They use Support Vector Machines (SVM) to evaluate the neighbor classifier against live data from the Gnutella (unstructured P2P network). And this method has a good impact in performance, scalability and resilience of P2P overlays. The same thing with [182], they propose a reinforcement learning-based query routing approach (RLQR). This approach seeks to reach high retrieval effectiveness as well as a lower search cost of resources in unstructured overlay, by introducing a new formulation for the K-Neighbors-Selection (K-NS) based on reinforcement learning. As a result, researchers are confronted with a strict line of study that needs extensive examination in order to establish the field of appropriate covering solutions.

\subsection{Bio-inspired P2P overlays}

SPIDER [183] is one of the countless variant of Bio inspired P2P overlays, like Ant colonies [184, 185], bee swarms [186]. It is a P2P structured overlay that is based on the spider web overlay who aims to emphasize the possible actions in this overlay like joining, leaving and lookups. So there are countless variants of bio-inspired algorithms that can improve P2P structured overlay to emphasize the possible actions in this overlay, like joining, leaving, and lookups, because these shortcomings are fairly impactful in this sort of overlays.

\subsection{Mobile Cloud, Fog and Edge computing in p2p overlays}

Cloud Computing has gained a vast size of scope in recent years. Therefore, it will be profitable if we combine cloud computing and a P2P network for information retrieval. The cloud layer improves the relevance of the retrieved information, distributing the indexing, and searching structures among its nodes. And the P2P layer uses a scalable implementation of trackers that monitor a set of peers sharing segments of data.

To overcome cloud computing shortcomings in terms of bandwidth, latency, and real-time response, it is suitable to deploy a Chord overlay into the fog layer.

Besides, we can establish another improvement by complementing edge computing with P2P overlay with a view to allow the end-user devices in VANET to communicate with each other and share computation.

\subsection{Blockchain P2P overlays}

Blockchain technology is the fruit of two emerged research areas, P2P computing and cryptography. Hence, there are various reasons to attract us to use blockchain over VANET. For instance, designing a new type of blockchain for secure message dissemination over VANET. 
Table 2: Open issues and future research directions.

\begin{tabular}{|c|c|c|c|}
\hline Open challenges & Problem(s) & Proposed solution(s) & References \\
\hline $\begin{array}{l}\text { Unmanned aerial vehi- } \\
\text { cle (UAV) based P2P } \\
\text { communications }\end{array}$ & $\begin{array}{l}\text { Frequent network disconnections, } \\
\text { collisions between UAVs. }\end{array}$ & $\begin{array}{l}\text { Set of UAVs hovers over VANETs } \\
\text { to bridge communication gaps be- } \\
\text { tween vehicles }\end{array}$ & $\begin{array}{ll}{[180,} & 184, \\
185, & 186, \\
187] & \end{array}$ \\
\hline $\begin{array}{l}\text { Enhancing P2P over- } \\
\text { lays with machine } \\
\text { learning techniques }\end{array}$ & $\begin{array}{l}\text { - Neighbors selection in self- } \\
\text { reorganizing networks. } \\
\text { - Cluster head selection in self- } \\
\text { reorganizing networks. } \\
\text { - Low retrieval effectiveness in the } \\
\text { unstructured overlay. } \\
\text { - High search cost of resources in } \\
\text { the unstructured overlay. }\end{array}$ & $\begin{array}{l}\text { - Propose a novel application of } \\
\text { machine learning. } \\
\text { - Introducing reinforcement } \\
\text { learning in routing. }\end{array}$ & $\begin{array}{l}{[181, \quad 182,} \\
188,189]\end{array}$ \\
\hline $\begin{array}{l}\text { Bio-inspired P2P over- } \\
\text { lays }\end{array}$ & $\begin{array}{l}\text { Overlay joining and leaving and In- } \\
\text { formation lookups. }\end{array}$ & $\begin{array}{l}\text { Improve P2P structured overlay } \\
\text { with bio-inspired algorithms. }\end{array}$ & $\begin{array}{ll}{[190,} & 191, \\
192]\end{array}$ \\
\hline $\begin{array}{l}\text { Mobile Cloud, Fog and } \\
\text { edge computing in p2p } \\
\text { overlays }\end{array}$ & $\begin{array}{l}\text { Low bandwidth, latency, and real- } \\
\text { time response. }\end{array}$ & $\begin{array}{l}\text { - Combine cloud computing and } \\
\text { a P2P network for information } \\
\text { retrieval. } \\
\text { - Deploy chord overlay in the fog } \\
\text { layer. } \\
\text { - Complementing edge computing } \\
\text { with P2P overlay. }\end{array}$ & $\begin{array}{l}{[193, \quad 194,} \\
195]\end{array}$ \\
\hline $\begin{array}{l}\text { Blockchain P2P over- } \\
\text { lays }\end{array}$ & Not secure message dissemination. & $\begin{array}{l}\text { Designing a new type of blockchain } \\
\text { for VANET }\end{array}$ & {$[196,197]$} \\
\hline $\begin{array}{l}\text { Wireless optical com- } \\
\text { munication in P2P } \\
\text { overlays }\end{array}$ & $\begin{array}{l}\text { Overlay joining and leaving. Infor- } \\
\text { mation retrieval. }\end{array}$ & $\begin{array}{l}\text { Use WOC in VANET to solve dis- } \\
\text { connections problems in structured } \\
\text { P2P overlay. }\end{array}$ & $\begin{array}{ll}{[198,} & 199, \\
200] & \end{array}$ \\
\hline New structured overlay & $\begin{array}{l}\text { Overlay joining, leaving and } \\
\text { lookup delay. }\end{array}$ & $\begin{array}{l}\text { Combining Pastry overlay and } \\
\text { AODV routing protocol }\end{array}$ & {$[201]$} \\
\hline Network coding & $\begin{array}{l}\text { system load, throughput limita- } \\
\text { tions }\end{array}$ & $\begin{array}{l}\text { Improve network performance, re- } \\
\text { duce network redundancy, and re- } \\
\text { duce latency using network coding }\end{array}$ & $\begin{array}{ll}{[198,} & 199, \\
200] & \end{array}$ \\
\hline $\begin{array}{l}\text { IEEE } 802.11 \mathrm{bd} \text { and } 5 \mathrm{G} \\
\text { V2X }\end{array}$ & $\begin{array}{l}\text { System load, latency, dependabil- } \\
\text { ity, and throughput constraints }\end{array}$ & $\begin{array}{l}\text { 5G NR-V2X can support P2P com- } \\
\text { munication requirements }\end{array}$ & $\begin{array}{l}{[202, \quad 203,} \\
204]\end{array}$ \\
\hline 6G in $\mathrm{P} 2 \mathrm{P}$ overlays & $\begin{array}{l}\text { - Low reliability, data rate, and } \\
\text { bandwidth. } \\
\text { - Power consumption and error } \\
\text { rate. }\end{array}$ & $\begin{array}{l}\text { Ensure the connectivity between } \\
\text { P2P nodes using } 6 \mathrm{G} \text {. }\end{array}$ & $\begin{array}{l}{[183, \quad 205,} \\
206,207]\end{array}$ \\
\hline
\end{tabular}

\subsection{Wireless optical communication in P2P overlays}

Wireless optical communication (WOC) is a nascent technology with high-speed connectivity [198] that uses an ultra-wide range of the unregulated spectrum that can reach 100 Gbps of data rate and provides a communication distance range that achieves more 
than 10,000 km [199]. Thereby, it is a promising solution to overcome traditional VANET limitation and allow p2p overlay to be established in the best manner.

\subsection{New structured overlay}

MADPastry [201] is a combination of Pastry overlay and AODV routing protocol at the network layer. MAD Pastry uses a set of keys that evenly divides the DHT key space so that neighboring nodes are physically close. This overlay facilitates the lookup process in Vanet applications like ITS due to the physically closest node which is more able to possess the information.

\subsection{Network coding}

Network coding technology integrates traditional routing and coding technologies and is mainly used in multicast networks. Applying network coding to VANETs can effectively improve network performance, reduce network redundancy, and reduce latency [208]. In order to further improve the reliability and real-time performance of V2R communications, researchers try to introduce network coding technology into data dissemination in VANETs [209]. Nevertheless, we should not overlook the unique concerns and obstacles that may occur while implementing network coding in a VANET, such as system load, latency, reliability, and throughput limitations. As a result, how to successfully implement P2P communications and network coding in VANETs remains an active area of study in the future.

\subsection{IEEE 802.11bd and 5G V2X}

$\mathrm{P} 2 \mathrm{P}$ systems on the VANET are focused on the application layer and allow vehicles to communicate directly without the assistance of a server. In the future, when more vehicles, people, and even their devices become connected, networks will need to adapt to increased system load, latency, dependability, and throughput constraints. Dedicated Short-Range Communications (DSRC) and Cellular V2X (C-V2X) communications may aid in improved traffic management, resulting in more environmentally friendly vehicles and cheaper fuel costs, as well as improved non-line-of-sight (NLOS) capabilities and increased resistance to interference[202]. However, if the quality of service (QoS) requirements for V2X use cases become increasingly demanding, as they are in a growing number of complex V2X applications [203]. Recent significant advancements in radio access technologies (RATs), such as 802.11 bd presented by the IEEE 802.11 Next-Generation V2X study group (NG $\mathrm{V} 2 \mathrm{X}$ ), and the next generation V2X technology (i.e., 5G NR-V2X), have been made to support the communication requirements of a wide variety of advanced vehicular applications [204]. Recent 5G V2X communication technology significantly boosts network capacity to handle a high number of users. As a result, researchers are confronted with a highly complex area of study that needs in-depth investigation in order to advance the field of P2P communications in VANET. 


\subsection{0. $6 G$ in P2P overlays}

Like 5G, the Sixth Generation (6G) mobile network has the ability to ensure the connectivity between P2P nodes, since 6G networks enable an enhanced reliability, data rate, and bandwidth while reducing power consumption, error rate, and communication time required for the mobile end-user [183, 206].

\section{Conclusion}

In recent years, VANET communications gained massive interest due to its wide range of uses and its variant of applications, including data sharing. Furthermore, the P2P overlay technique is one of the essential data-sharing applications since each method has its own different set of qualities, advantages, and specifications. In this extensive survey, we have made the following significant contributions to clarify the differences between these applications:

- We have presented P2P networks and VANETs.

- We have proposed a novel taxonomy that included the majority of P2P overlay methods for VANET, as well as their applications.

- We have classified the various P2P overlay techniques for VANET into three categories that are the most studied in the literature.

- We have also discussed cutting-edge technologies and protocols for P2P approaches in VANETs, as well as crucial challenges that affect their performance.

- Moreover, for each approach, we have identified the issues, applications, and key open challenges. Furthermore, we mainly explored its requirements, its primary objective, and its advantages and drawbacks.

A comparative study is performed among the discussed techniques, focusing on the lookup process and download time. After describing all these $\mathrm{P} 2 \mathrm{P}$ overlay schemes and techniques along with their learned lessons and open research challenges, it is natural to review the fundamental difficulties that are only apparently addressed in the literature. Indeed, each P2P proposal has several tough challenges and flaws that demand additional examination, but these are usually left as future works or scarcely looked at by scientists. For example, each technique should carefully consider the various limits of using a P2P overlay and how to overcome them. Also, to avoid destabilization of P2P overlays, the node mobility and the network load have to be considered. This allows us to outline future research perspectives for researchers interested in digging further into the P2P overlay in VANET. To summarize, the $\mathrm{P} 2 \mathrm{P}$ overlay mechanism in VANET is still in its immaturity and requires further exploration to achieve a comprehensive level of research. As future research, we are currently planning to investigate deeply the concept of artificial intelligence, $5 \mathrm{G}$, and UAVs and how we can use it in a $\mathrm{P} 2 \mathrm{P}$ overlay. 


\section{Acknowledgement}

This research was partially supported by grant \# G00003280 from the UAE University's National Space Science and Technology Center.

\section{References}

[1] M. Singh, C. Kumar, P. Nath, Challenges and protocols for p2p applications in multi-hop wireless networks, in: 2018 Second International Conference on Computing Methodologies and Communication (ICCMC), 2018, pp. 310-316. doi:10.1109/ICCMC.2018.8487911.

[2] I. Woungang, F. Tseng, Y. Lin, L. Chou, H. Chao, M. S. Obaidat, Mr-chord: Improved chord lookup performance in structured mobile p2p networks, IEEE Systems Journal 9 (3) (2015) 743-751. doi: 10.1109/JSYST . 2014.2306147.

[3] J. Rybicki, B. Scheuermann, W. Kiess, C. Lochert, P. Fallahi, M. Mauve, Challenge: Peers on wheels - a road to new traffic information systems, in: Proceedings of the 13th Annual ACM International Conference on Mobile Computing and Networking, MobiCom '07, ACM, New York, NY, USA, 2007, pp. 215-221. doi:10.1145/1287853.1287879.

URL http://doi.acm.org/10.1145/1287853.1287879

[4] C. Englund, L. Chen, A. Vinel, S. Y. Lin, Future applications of vanets, in: Vehicular ad hoc Networks, Springer, 2015, pp. 525-544. doi:10.1007/978-3-319-15497-8_18.

URL https://doi.org/10.1007/978-3-319-15497-8.18

[5] J. Wang, Y. Shao, Y. Ge, R. Yu, A survey of vehicle to everything (v2x) testing, Sensors 19 (2) (2019). doi:10.3390/s19020334.

URL https://www.mdpi.com/1424-8220/19/2/334

[6] J. Jeong, Y. Shen, T. Oh, S. Céspedes, N. Benamar, M. Wetterwald, J. Härri, A comprehensive survey on vehicular networks for smart roads: A focus on ip-based approaches, Vehicular Communications 29 (2021) 100334. doi:https://doi.org/10.1016/j.vehcom.2021.100334.

URL https://www.sciencedirect.com/science/article/pii/S2214209621000036

[7] H. Kim, S. Hong, J. Kim, J. Ryou, Intelligent application protection mechanism for transportation in v2c environment, IEEE Access 8 (2020) 86777-86787. doi:10.1109/ACCESS .2020.2991273.

[8] M. Hamad, E. Regnath, J. Lauinger, V. Prevelakis, S. Steinhorst, Spps: Secure policy-based publish/subscribe system for v2c communication, in: 2021 Design, Automation Test in Europe Conference Exhibition (DATE), 2021, pp. 529-534. doi:10.23919/DATE51398.2021.9474070.

[9] A. Ghosal, M. Conti, Security issues and challenges in v2x: A survey, Computer Networks 169 (2020) 107093. doi:https://doi.org/10.1016/j.comnet.2019.107093.

URL https://www.sciencedirect.com/science/article/pii/S1389128619305857

[10] J. T. J. Penttinen, Services and Applications, Wiley, 2019, Ch. 7, pp. 187-203. doi:10.1002/ $9781119275695 \cdot \operatorname{ch} 7$.

[11] W. Anwar, N. Franchi, G. Fettweis, Physical layer evaluation of v2x communications technologies: $5 \mathrm{~g}$ nr-v2x, lte-v2x, ieee 802.11bd, and ieee 802.11p, in: 2019 IEEE 90th Vehicular Technology Conference (VTC2019-Fall), 2019, pp. 1-7. doi:10.1109/VTCFall.2019.8891313.

[12] A. Alalewi, I. Dayoub, S. Cherkaoui, On 5g-v2x use cases and enabling technologies: A comprehensive survey, IEEE Access 9 (2021) 107710-107737. doi:10.1109/ACCESS. 2021.3100472.

[13] S. Abdel Hakeem, 5g-v2x: Standardization, architecture, use cases, network-slicing, and edgecomputing, Wireless Networks 26 (11 2020). doi:10.1007/s11276-020-02419-8.

[14] H. Hartenstein, H. Hartenstein, K. Laberteaux, VANET : vehicular applications and inter-networking technologies, Chichester, U.K. : Wiley, 2010, includes index.

[15] L. Aparecido, Data dissemination in vehicular networks: Challenges, solutions, and future perspectives, in: 2015 7th International Conference on New Technologies, Mobility and Security (NTMS), 2015, pp. 1-5. doi:10.1109/NTMS.2015.7266482. 
[16] O. K. Tonguz, N. Wisitpongphan, F. Bai, Dv-cast: A distributed vehicular broadcast protocol for vehicular ad hoc networks, IEEE Wireless Communications 17 (2) (2010) 47-57. doi:10.1109/MWC. 2010.5450660 .

[17] L. A. Villas, T. P. C. de Andrade, N. L. S. da Fonseca, An efficient and robust protocol to disseminate data in highway environments with different traffic conditions, in: 2014 IEEE Symposium on Computers and Communications (ISCC), 2014, pp. 1-6. doi:10.1109/ISCC.2014.6912605.

[18] Y. S. Chen, Y. W. Lin, S. L. Lee, A mobicast routing protocol with carry-and-forward in vehicular ad-hoc networks, in: 2010 5th International ICST Conference on Communications and Networking in China, 2010, pp. 1-5.

[19] Y. W. L. Y. S. Chen, S. L. Lee, A mobicast routing protocol in vehicular ad-hoc networks, in: GLOBECOM 2009 - 2009 IEEE Global Telecommunications Conference, 2009, pp. 1-6. doi:10. 1109/GLOCOM. 2009.5426207.

[20] M. Bakhouya, J. Gaber, P. Lorenz, An adaptive approach for information dissemination in vehicular ad hoc networks, J. Netw. Comput. Appl. 34 (6) (2011) 1971-1978. doi:10.1016/j.jnca.2011.06.010. URL http://dx.doi.org/10.1016/j.jnca.2011.06.010

[21] L. A. Villas, A. Boukerche, R. B. Araujo, A. A. F. Loureiro, J. Ueyama, Network partition-aware geographical data dissemination, in: 2013 IEEE International Conference on Communications (ICC), 2013, pp. 1439-1443. doi:10.1109/ICC.2013.6654713.

[22] E. K. Lua, J. Crowcroft, M. Pias, R. Sharma, S. Lim, A survey and comparison of peer-to-peer overlay network schemes, IEEE Communications Surveys Tutorials 7 (2) (2005) 72-93. doi:10.1109/COMST. 2005. 1610546.

[23] Napster, https://fr.napster.com/, accessed: 2015-09-30 (1999).

[24] I. Stoica, R. Morris, D. Karger, M. F. Kaashoek, H. Balakrishnan, Chord: A scalable peer-to-peer lookup service for internet applications, SIGCOMM Comput. Commun. Rev. 31 (4) (2001) 149-160. doi: 10.1145/964723.383071.

URL http://doi.acm.org/10.1145/964723.383071

[25] S. Ratnasamy, P. Francis, M. Handley, R. Karp, S. Shenker, A scalable content-addressable network, ACM SIGCOMM Computer Communication Review 31 (09 2001). doi:10.1145/383059.383072.

[26] B. B. Yang, H. Garcia-Molina, Designing a super-peer network, in: Proceedings 19th International Conference on Data Engineering (Cat. No.03CH37405), 2003, pp. 49-60. doi:10.1109/ICDE. 2003. 1260781.

[27] N. S. Good, A. Krekelberg, Usability and privacy: A study of kazaa p2p file-sharing, in: Proceedings of the SIGCHI Conference on Human Factors in Computing Systems, CHI '03, ACM, New York, NY, USA, 2003, pp. 137-144. doi:10.1145/642611.642636.

URL http://doi.acm.org/10.1145/642611.642636

[28] H. Che, Review: From p2p to web services and grids: Peers in a client/server world, The Computer Journal 48 (3) (2005) 379-379. doi:10.1093/comjnl/bxh081.

[29] R. F. C. Gnutella, The Gnutella Protocol Specification v0.4 (2004).

[30] I. Lujic, V. D. Maio, K. Pollhammer, I. Bodrozic, J. Lasic, I. Brandic, Increasing traffic safety with real-time edge analytics and $5 \mathrm{~g}$, in: Proceedings of the 4th International Workshop on Edge Systems, Analytics and Networking, EdgeSys '21, Association for Computing Machinery, New York, NY, USA, 2021, p. 19-24. doi:10.1145/3434770.3459732.

URL https://doi.org/10.1145/3434770.3459732

[31] K. Yu, L. Lin, M. Alazab, L. Tan, B. Gu, Deep learning-based traffic safety solution for a mixture of autonomous and manual vehicles in a 5 g-enabled intelligent transportation system, IEEE Transactions on Intelligent Transportation Systems 22 (7) (2021) 4337-4347. doi:10.1109/TITS.2020.3042504.

[32] T. Ojanperä, J. Mäkelä, O. Mämmelä, M. Majanen, O. Martikainen, Use cases and communications architecture for 5g-enabled road safety services, in: 2018 European Conference on Networks and Communications (EuCNC), 2018, pp. 335-340. doi:10.1109/EuCNC.2018.8443193.

[33] T. Ojanperä, M. Kutila, P. Pyykönen, J. Scholliers, T. Sukuvaara, K. Mäenpää, O. Huuskonen, Development and piloting of novel 5g-enabled road safety services, in: 2019 IEEE Wireless Communi- 
cations and Networking Conference Workshop (WCNCW), 2019, pp. 1-6. doi:10.1109/WCNCW. 2019. 8902858.

[34] H. Khan, P. Luoto, M. Bennis, M. Latva-aho, On the application of network slicing for 5g-v2x, in: European Wireless 2018; 24th European Wireless Conference, 2018, pp. 1-6.

[35] O. S. Oubbati, A. Lakas, F. Zhou, M. Güneş, M. B. Yagoubi, A survey on position-based routing protocols for flying ad hoc networks (fanets), Vehicular Communications 10 (2017) 29-56. doi: https://doi.org/10.1016/j.vehcom.2017.10.003.

URL https://www.sciencedirect.com/science/article/pii/S2214209617300529

[36] D. Shumeye Lakew, U. Sa'ad, N.-N. Dao, W. Na, S. Cho, Routing in flying ad hoc networks: A comprehensive survey, IEEE Communications Surveys Tutorials 22 (2) (2020) 1071-1120. doi:10. 1109/COMST . 2020. 2982452.

[37] O. S. Oubbati, M. Atiquzzaman, P. Lorenz, M. H. Tareque, M. S. Hossain, Routing in flying ad hoc networks: Survey, constraints, and future challenge perspectives, IEEE Access 7 (2019) 81057-81105. doi:10.1109/ACCESS. 2019.2923840.

[38] M. A. Khan, A. Safi, I. M. Qureshi, I. U. Khan, Flying ad-hoc networks (fanets): A review of communication architectures, and routing protocols, in: 2017 First International Conference on Latest trends in Electrical Engineering and Computing Technologies (INTELLECT), 2017, pp. 1-9. doi: 10.1109/INTELLECT . 2017.8277614.

[39] F. Noor, M. A. Khan, A. Al-Zahrani, I. Ullah, K. A. Al-Dhlan, A review on communications perspective of flying ad-hoc networks: Key enabling wireless technologies, applications, challenges and open research topics, Drones 4 (4) (2020). doi:10.3390/drones4040065.

URL https ://www.mdpi.com/2504-446X/4/4/65

[40] Q. Sang, H. Wu, L. Xing, P. Xie, Review and comparison of emerging routing protocols in flying ad hoc networks, Symmetry 12 (6) (2020). doi:10.3390/sym12060971.

URL https : //www.mdpi.com/2073-8994/12/6/971

[41] A. Guillen-Perez, M.-D. Cano, Flying ad hoc networks: A new domain for network communications, Sensors 18 (2018) 3571. doi:10.3390/s18103571.

[42] R. Hussain, J. Lee, S. Zeadally, Trust in vanet: A survey of current solutions and future research opportunities, IEEE Transactions on Intelligent Transportation Systems 22 (5) (2021) 2553-2571. doi:10.1109/TITS.2020.2973715.

[43] A. Srivastava, A. Prakash, R. Tripathi, Location based routing protocols in vanet: Issues and existing solutions, Vehicular Communications 23 (2020) 100231. doi:https://doi.org/10.1016/j.vehcom. 2020.100231.

URL https://www.sciencedirect.com/science/article/pii/S2214209620300024

[44] R. Hussain, F. Hussain, S. Zeadally, Integration of vanet and $5 \mathrm{~g}$ security: A review of design and implementation issues, Future Generation Computer Systems 101 (2019) 843-864. doi:https:// doi.org/10.1016/j.future.2019.07.006.

URL https://www.sciencedirect.com/science/article/pii/S0167739X19306909

[45] F. Benabdallah, A. Hamza, M. Bechrif, Simulation and analysis of vanets performances based on the choice of mobility model, in: 2017 Computing Conference, 2017, pp. 1238-1242. doi:10.1109/SAI. 2017.8252248.

[46] J. Tian, F. Meng, Comparison survey of mobility models in vehicular ad-hoc network (vanet), in: 2020 IEEE 3rd International Conference on Automation, Electronics and Electrical Engineering (AUTEEE), 2020, pp. 337-342. doi:10.1109/AUTEEE50969.2020.9315583.

[47] N. Jahangeer, P. B, H. Aldabbas, A study on modeling vehicles mobility with mlc for enhancing vehicle-to-vehicle connectivity in vanet, Journal of Ambient Intelligence and Humanized Computing (08 2021). doi:10.1007/s12652-020-02559-x.

[48] F. Benabdallah, A. Hamza, M. Bechrif, Simulation and analysis of vanets performances based on the choice of mobility model, in: 2017 Computing Conference, 2017, pp. 1238-1242. doi:10.1109/SAI. 2017.8252248.

[49] J. Tian, F. Meng, Comparison survey of mobility models in vehicular ad-hoc network (vanet), in: 
2020 IEEE 3rd International Conference on Automation, Electronics and Electrical Engineering (AUTEEE), 2020, pp. 337-342. doi:10.1109/AUTEEE50969.2020.9315583.

[50] W. Anwar, N. Franchi, G. Fettweis, Physical layer evaluation of v2x communications technologies: 5g nr-v2x, lte-v2x, ieee 802.11bd, and ieee 802.11p, in: 2019 IEEE 90th Vehicular Technology Conference (VTC2019-Fall), 2019, pp. 1-7. doi:10.1109/VTCFall.2019.8891313.

[51] J. Wang, Y. Shao, Y. Ge, R. Yu, A survey of vehicle to everything (v2x) testing, Sensors 19 (2019) 334. doi:10.3390/s19020334.

[52] D. Milic, S. Vasić, N. Petrovic, S. Suljović, V. Nyangaresi, Outage probability of a simulated smartcity $5 \mathrm{~g}$ mimo system with l-branch sc receiver undergoing $\mathrm{k}-\mu$ fading and nakagami-m interference, in: XV International SAUM Conference, 2021, pp. 13-16.

[53] J. Zhang, S. Chen, Y. Lin, J. Zheng, B. Ai, L. Hanzo, Cell-free massive mimo: A new next-generation paradigm, IEEE Access 7 (2019) 99878-99888. doi:10.1109/ACCESS. 2019.2930208.

[54] G. Artner, W. Kotterman, G. Del Galdo, M. A. Hein, Automotive antenna roof for cooperative connected driving, IEEE Access 7 (2019) 20083-20090. doi:10.1109/ACCESS . 2019. 2897219.

[55] M. Kumar, V. Nath, Introducing multiband and wideband microstrip patch antennas using fractal geometries: Development in last decade, Wireless Personal Communications 98 (01 2018). doi: 10.1007/s11277-017-4965-x.

[56] R. Vijay, R. Rao T, Penta-band linear tapered feed spiral antenna design and radio link characterization for vehicular communications, Wireless Personal Communications 96 (2017) 1-18. doi: 10.1007/s11277-017-4341-x.

[57] R. Vijay, R. Rao T, Design and performance analysis of a penta-band spiral antenna for vehicular communications, Wireless Personal Communications 96 (10 2017). doi:10.1007/s11277-017-4064-z.

[58] B. Madhav, A. Tirunagari, S. Kotamraju, Transparent and conformal wheel-shaped fractal antenna for vehicular communication applications, AEU - International Journal of Electronics and Communications 91 (04 2018). doi:10.1016/j.aeue.2018.04.028.

[59] P. Dong, T. Zheng, S. Yu, H. Zhang, X. Yan, Enhancing vehicular communication using 5g-enabled smart collaborative networking, IEEE Wireless Communications 24 (6) (2017) 72-79. doi:10.1109/ MWC. 2017.1600375.

[60] M. S. Sharawi, M. Ikram, A. Shamim, A two concentric slot loop based connected array mimo antenna system for $4 \mathrm{~g} / 5 \mathrm{~g}$ terminals, IEEE Transactions on Antennas and Propagation 65 (12) (2017) 66796686. doi:10.1109/TAP. 2017.2671028.

[61] J. Zhao, J. Liu, J. Jiang, F. Gao, Efficient deployment with geometric analysis for mmwave uav communications, IEEE Wireless Communications Letters 9 (7) (2020) 1115-1119. doi:10.1109/LWC. 2020.2982637.

[62] M. Alrabeiah, A. Alkhateeb, Deep learning for mmwave beam and blockage prediction using sub-6 ghz channels, IEEE Transactions on Communications 68 (9) (2020) 5504-5518. doi:10.1109/TCOMM. 2020.3003670 .

[63] S. H. Dokhanchi, B. S. Mysore, K. V. Mishra, B. Ottersten, A mmwave automotive joint radarcommunications system, IEEE Transactions on Aerospace and Electronic Systems 55 (3) (2019) 12411260. doi:10.1109/TAES.2019.2899797.

[64] Z. Li, T. Yu, R. Fukatsu, G. K. Tran, K. Sakaguchi, Towards safe automated driving: Design of software-defined dynamic mmwave v2x networks and poc implementation, IEEE Open Journal of Vehicular Technology 2 (2021) 78-93. doi:10.1109/0JVT.2021.3049783.

[65] C. Tripp-Barba, A. Zaldívar-Colado, L. Urquiza-Aguiar, J. A. Aguilar-Calderón, Survey on routing protocols for vehicular ad hoc networks based on multimetrics, Electronics 8 (10) (2019). doi:10. 3390/electronics8101177.

URL https://www .mdpi.com/2079-9292/8/10/1177

[66] S. Saxena Rahul; Jain Monika; Sharma, D.P.; Jaidka, A review on vanet routing protocols and proposing a parallelized genetic algorithm based heuristic modification to mobicast routing for real time message passing, journal of intelligent and fuzzy systems (01 2019). doi:10.3233/JIFS-169950.

[67] R. A. Nazib, S. Moh, Reinforcement learning-based routing protocols for vehicular ad hoc networks: 
A comparative survey, IEEE Access 9 (2021) 27552-27587. doi:10.1109/ACCESS. 2021.3058388.

[68] C. E. Perkins, E. M. Royer, Ad-hoc on-demand distance vector routing, in: Mobile Computing Systems and Applications, 1999. Proceedings. WMCSA '99. Second IEEE Workshop on, 1999, pp. 90-100. doi:10.1109/MCSA.1999.749281.

[69] D. A. Johnson, David B.and Maltz, Dynamic Source Routing in Ad Hoc Wireless Networks, Springer US, Boston, MA, 1996, Ch. 5, pp. 153-181. doi:10.1007/978-0-585-29603-6_5.

URL https://doi.org/10.1007/978-0-585-29603-6_5

[70] X. Shen, Y. Wu, Z. Xu, X. Lin, Aodv-pnt: An improved version of aodv routing protocol with predicting node trend in vanet, in: The 7th IEEE/International Conference on Advanced Infocomm Technology, 2014, pp. 91-97. doi:10.1109/ICAIT.2014.7019536.

[71] X. Peng, Y. Wu, Z. Xu, X. Lin, Aodv-mr: Aodv with multi-rrep for vanet, in: The 7th IEEE/International Conference on Advanced Infocomm Technology, 2014, pp. 172-176. doi:10. 1109/ICAIT. 2014.7019549.

[72] C. Sottile, A. F. Santamaria, S. Marano, A reactive routing protocol for vanets based on composite metric concept, in: International Symposium on Performance Evaluation of Computer and Telecommunication Systems (SPECTS 2014), 2014, pp. 642-646. doi:10.1109/SPECTS.2014.6880005.

[73] K. Manel, C. Lamia, Sodv speed based ad hoc on demand vector link routing protocol: A routing protocol for vanet networks, in: 2017 Sixth International Conference on Communications and Networking (ComNet), 2017, pp. 1-8. doi:10.1109/COMNET.2017.8285587.

[74] M. Valantina, S. Jayashri, Q-learning based point to point data transfer in vanets, Procedia Computer Science 57 (2015) 1394-1400. doi:10.1016/j.procs.2015.07.456.

[75] C. Wu, S. Ohzahata, T. Kato, Flexible, portable, and practicable solution for routing in vanets: A fuzzy constraint q-learning approach, IEEE Transactions on Vehicular Technology 62 (9) (2013) 4251-4263. doi:10.1109/TVT.2013. 2273945.

[76] J. Wu, M. Fang, X. li, Reinforcement learning based mobility adaptive routing for vehicular ad-hoc networks, Wireless Personal Communications 101 (08 2018). doi:10.1007/s11277-018-5809-z.

[77] D. Zhang, T. Zhang, X. Liu, Novel self-adaptive routing service algorithm for application in vanet, Applied Intelligence 49 (05 2019). doi:10.1007/s10489-018-1368-y.

[78] C. Wu, K. Kumekawa, T. Kato, Distributed reinforcement learning approach for vehicular ad hoc networks, IEICE Transactions 93-B (2010) 1431-1442. doi:10.1587/transcom.E93.B.1431.

[79] C. Wu, Y. Ji, F. Liu, S. Ohzahata, T. Kato, Toward practical and intelligent routing in vehicular ad hoc networks, IEEE Transactions on Vehicular Technology 64 (12) (2015) 5503-5519. doi:10.1109/ TVT. 2015.2481464.

[80] X. Yang, W. Zhang, H. Lu, L. Zhao, V2v routing in vanet based on heuristic q-learning, INTERNATIONAL JOURNAL OF COMPUTERS COMMUNICATIONS and CONTROL 15 (07 2020). doi:10.15837/ijccc.2020.5.3928.

[81] S. Nithya, G. A. Kumar, P. Adhavan, Destination-sequenced distance vector routing (dsdv) using clustering approach in mobile adhoc network, in: 2012 International Conference on Radar, Communication and Computing (ICRCC), 2012, pp. 319-323. doi:10.1109/ICRCC.2012.6450604.

[82] P. Jacquet, P. Muhlethaler, T. Clausen, A. Laouiti, A. Qayyum, L. Viennot, Optimized link state routing protocol for ad hoc networks, in: Proceedings. IEEE International Multi Topic Conference, 2001. IEEE INMIC 2001. Technology for the 21st Century., 2001, pp. 62-68. doi:10.1109/INMIC. 2001.995315.

[83] T.-W. Chen, M. Gerla, Global state routing: a new routing scheme for ad-hoc wireless networks, in: Communications, 1998. ICC 98. Conference Record. 1998 IEEE International Conference on, Vol. 1, 1998, pp. 171-175 vol.1. doi:10.1109/ICC.1998.682615.

[84] W.-H. Kuo, S. Dong, J.-S. Huang, Pheromone-based v2v unicast routing scheme in vanets, in: 2013 International Conference on Connected Vehicles and Expo (ICCVE), 2013, pp. 783-784. doi:10. 1109/ICCVE. 2013.6799896.

[85] A. Boukerche, B. Turgut, N. Aydin, M. Z. Ahmad, L. Bölöni, D. Turgut, Routing protocols in ad hoc networks: A survey, Computer Networks 55 (13) (2011) 3032 - 3080. doi:https://doi .org/10. 
1016/j.comnet.2011.05.010.

URL http://www.sciencedirect.com/science/article/pii/S1389128611001654

[86] A. Zafar, An overview of zone routing protocol, International Journal of Advances in Science and Technology 3 (02 2012).

[87] P. B. Key, G. A. Cope, Distributed dynamic routing schemes, IEEE Communications Magazine 28 (10) (1990) 54-58. doi:10.1109/35.60367.

[88] X. Ji, W. Xu, C. Zhang, T. Yun, G. Zhang, X. Wang, Y. Wang, B. Liu, Keep forwarding path freshest in vanet via applying reinforcement learning, in: 2019 IEEE First International Workshop on Network Meets Intelligent Computations (NMIC), 2019, pp. 13-18. doi:10.1109/NMIC. 2019.00008.

[89] R. Li, F. Li, X. Li, Y. Wang, Qgrid: Q-learning based routing protocol for vehicular ad hoc networks, in: 2014 IEEE 33rd International Performance Computing and Communications Conference (IPCCC), 2014, pp. 1-8. doi:10.1109/PCCC.2014.7017079.

[90] P.-J. Chuang, M.-C. Liu, Advanced junction-based routing in vehicular ad-hoc networks, in: 2015 9th International Conference on Future Generation Communication and Networking (FGCN), 2015, pp. 17-20. doi:10.1109/FGCN.2015.17.

[91] A. Soua, H. Afifi, Adaptive data collection protocol using reinforcement learning for vanets, in: 2013 9th International Wireless Communications and Mobile Computing Conference (IWCMC), 2013, pp. 1040-1045. doi:10.1109/IWCMC.2013.6583700.

[92] M. Saravanan, P. Ganeshkumar, Routing using reinforcement learning in vehicular ad hoc networks, Computational Intelligence 36 (01 2020). doi:10.1111/coin.12261.

[93] J. Na, C. kwon Kim, Glr: A novel geographic routing scheme for large wireless ad hoc networks, Computer Networks 50 (2006) 3225-3522.

[94] Y.-B. Ko, N. H. Vaidya, Location-aided routing (lar) in mobile ad hoc networks, in: Proceedings of the 4th Annual ACM/IEEE International Conference on Mobile Computing and Networking, MobiCom '98, ACM, New York, NY, USA, 1998, pp. 66-75. doi:10.1145/288235.288252. URL http://doi.acm.org/10.1145/288235.288252

[95] S. Basagni, I. Chlamtac, V. R. Syrotiuk, B. A. Woodward, A distance routing effect algorithm for mobility (dream), in: Proceedings of the 4th Annual ACM/IEEE International Conference on Mobile Computing and Networking, MobiCom '98, ACM, New York, NY, USA, 1998, pp. 76-84. doi: $10.1145 / 288235.288254$.

URL http://doi.acm.org/10.1145/288235.288254

[96] N. Dharani Kumari, B. Shylaja, Amgrp: Ahp-based multimetric geographical routing protocol for urban environment of vanets, Journal of King Saud University - Computer and Information Sciences 31 (1) (2019) 72-81. doi:https://doi.org/10.1016/j.jksuci.2017.01.001.

URL https://www.sciencedirect.com/science/article/pii/S1319157817300095

[97] X. Wang, W. Liu, L. Yang, W. Zhang, C. Peng, A new content-centric routing protocol for vehicular ad hoc networks, in: 2016 22nd Asia-Pacific Conference on Communications (APCC), 2016, pp. 552-558. doi:10.1109/APCC. 2016.7581473.

[98] R. Kasana, S. Kumar, Multimetric next hop vehicle selection for geocasting in vehicular ad-hoc networks, in: 2015 IEEE International Conference on Computational Intelligence Communication Technology, 2015, pp. 400-405. doi:10.1109/CICT.2015.150.

[99] H. Yu, J. Yoo, S. Ahn, A vanet routing based on the real-time road vehicle density in the city environment, in: 2013 Fifth International Conference on Ubiquitous and Future Networks (ICUFN), 2013, pp. 333-337. doi:10.1109/ICUFN.2013.6614836.

[100] X. Li, C. Chen, Z. Wang, L. Liu, J. Lv, An adaptive geographic routing protocol based on quality of transmission in urban vanets, in: 2018 IEEE International Conference on Smart Internet of Things (SmartIoT), 2018, pp. 52-57. doi:10.1109/SmartIoT.2018.00019.

[101] T. Nebbou, M. Lehsaini, Greedy curvemetric-based routing protocol for vanets, in: 2018 International Conference on Selected Topics in Mobile and Wireless Networking (MoWNeT), 2018, pp. 1-6. doi: 10.1109/MoWNet.2018.8428952.

[102] C. Bouras, V. Kapoulas, E. Tsanai, A gpsr enhancement mechanism for routing in vanets, in: M. C. 
Aguayo-Torres, G. Gómez, J. Poncela (Eds.), Wired/Wireless Internet Communications, Springer International Publishing, Cham, 2015, pp. 94-107.

[103] X. Yang, M. Li, Z. Qian, T. Di, Improvement of gpsr protocol in vehicular ad hoc network, IEEE Access 6 (2018) 39515-39524. doi:10.1109/ACCESS .2018.2853112.

[104] Y. Jin, Q. Pei, N. Zhang, A connectivity-aware intersection-based routing in vanets, EURASIP Journal on Wireless Communications and Networking 2014 (2014) 42. doi:10.1186/1687-1499-2014-42.

[105] K. Qureshi, F. Hussain, H. Abdullah, Distance and signal quality aware next hop selection routing protocol for vehicular ad hoc networks, Neural Computing and Applications 32 (04 2020). doi: 10.1007/s00521-019-04320-8.

[106] J. Wu, M. Fang, H. Li, X. Li, Rsu-assisted traffic-aware routing based on reinforcement learning for urban vanets, IEEE Access 8 (2020) 5733-5748. doi:10.1109/ACCESS. 2020.2963850.

[107] S. Yanglong, Y. Lin, Y. Tang, A Reinforcement Learning-Based Routing Protocol in VANETs, Springer, 2019, Ch. 25, pp. 2493-2500. doi:10.1007/978-981-10-6571-2_303.

[108] B.-S. Roh, M.-H. Han, J.-H. Ham, K.-I. Kim, Q-lbr: Q-learning based load balancing routing for uav-assisted vanet, Sensors 20 (19) (2020). doi:10.3390/s20195685.

URL https://www.mdpi.com/1424-8220/20/19/5685

[109] S. . Lee, M. Gerla, Split multipath routing with maximally disjoint paths in ad hoc networks, in: ICC 2001. IEEE International Conference on Communications. Conference Record (Cat. No.01CH37240), Vol. 10, 2001, pp. 3201-3205 vol.10. doi:10.1109/ICC.2001.937262.

[110] M. K. Marina, S. R. Das, On-demand multipath distance vector routing in ad hoc networks, in: Proceedings Ninth International Conference on Network Protocols. ICNP 2001, 2001, pp. 14-23. doi: 10.1109/ICNP. 2001.992756.

[111] P. Guangyu, M. Geria, X. Hong, Lanmar: landmark routing for large scale wireless ad hoc networkswith group mobility, in: 2000 First Annual Workshop on Mobile and Ad Hoc Networking and Computing. MobiHOC (Cat. No.00EX444), 2000, pp. 11-18. doi:10.1109/MOBHOC. 2000.869208.

[112] P. Sinha, R. Sivakumar, V. Bharghavan, Cedar: a core-extraction distributed ad hoc routing algorithm, in: IEEE INFOCOM '99. Conference on Computer Communications. Proceedings. Eighteenth Annual Joint Conference of the IEEE Computer and Communications Societies. The Future is Now (Cat. No.99CH36320), Vol. 1, 1999, pp. 202-209 vol.1. doi:10.1109/INFCOM.1999.749269.

[113] N. Kumar, N. Chilamkurti, J. Park, Alca: Agent learning-based clustering algorithm in vehicular ad hoc networks, Personal and Ubiquitous Computing 17 (12 2013). doi:10.1007/s00779-012-0600-8.

[114] Y. Ji, C. Wu, T. Yoshinaga, Context-aware unified routing for vanets based on virtual clustering, in: 2016 IEEE 27th Annual International Symposium on Personal, Indoor, and Mobile Radio Communications (PIMRC), 2016, pp. 1-6. doi:10.1109/PIMRC.2016.7794599.

[115] X. Bi, D. Gao, M. Yang, A reinforcement learning-based routing protocol for clustered ev-vanet, in: 2020 IEEE 5th Information Technology and Mechatronics Engineering Conference (ITOEC), 2020, pp. 1769-1773. doi:10.1109/ITOEC49072.2020.9141805.

[116] C. Wu, T. Yoshinaga, Y. Ji, Y. Zhang, Computational intelligence inspired data delivery for vehicleto-roadside communications, IEEE Transactions on Vehicular Technology 67 (12) (2018) 12038-12048. doi:10.1109/TVT.2018.2871606.

[117] A. Nahar, D. Das, Adaptive reinforcement routing in software defined vehicular networks, in: 2020 International Wireless Communications and Mobile Computing (IWCMC), 2020, pp. 2118-2123. doi: 10.1109/IWCMC48107.2020.9148237.

[118] H. Joshi, M. Sichitiu, M. Kihl, Distributed robust geocast multicast routing for inter-vehicle communication, Proceedings of WEIRD Workshop on WiMax, Wireless and Mobility (05 2007).

[119] and, and, A cluster-based directional routing protocol in vanet, in: 2010 IEEE 12th International Conference on Communication Technology, 2010, pp. 1172-1175. doi:10.1109/ICCT.2010.5689132.

[120] M. Durresi, A. Durresi, L. Barolli, Emergency broadcast protocol for inter-vehicle communications, in: 11th International Conference on Parallel and Distributed Systems (ICPADS'05), Vol. 2, 2005, pp. 402-406. doi:10.1109/ICPADS.2005.147.

[121] Z. L, C. G, L. H, L. D, W. Z, Nppb: A broadcast scheme in dense vanets, Information Technology 
Journal 9 (02 2010). doi:10.3923/itj.2010.247.256.

[122] K. C. Lee, S.-h. Lee, R. Cheung, U. Lee, M. Gerla, First experience with cartorrent in a real vehicular ad hoc network testbed, in: 2007 Mobile Networking for Vehicular Environments, 2007, pp. 109-114. doi:10.1109/MOVE. 2007.4300814.

[123] L. Chisalita, N. Shahmehri, A peer-to-peer approach to vehicular communication for the support of traffic safety applications, in: Proceedings. The IEEE 5th International Conference on Intelligent Transportation Systems, 2002, pp. 336-341. doi:10.1109/ITSC.2002.1041239.

[124] H. Balakrishnan, M. F. Kaashoek, D. Karger, R. Morris, I. Stoica, Looking up data in p2p systems, Commun. ACM 46 (2) (2003) 43-48. doi:10.1145/606272.606299. URL http://doi.acm.org/10.1145/606272.606299

[125] a. J. M.-G. C. Caballero-Gil, P. Caballero-Gil, Using groups to reduce communication overhead in vanets, in: The Second International Conference on Advances in P2P Systems, 2010, pp. 26-31.

[126] L. Yang, T. Mo, H. Li, Research on v2v communication based on peer to peer network, in: 2018 International Conference on Intelligent Autonomous Systems (ICoIAS), 2018, pp. 105-110. doi: 10.1109/ICoIAS. 2018.8494142.

[127] S. Ono, F. Kikuchi, T. Yamazaki, T. Miyoshi, K. Sezaki, An information sharing method using p2p and v2v communications in vanet, in: 2020 IEEE International Conference on Consumer Electronics - Taiwan (ICCE-Taiwan), 2020, pp. 1-2. doi:10.1109/ICCE-Taiwan49838.2020.9258348.

[128] T. Miyoshi, Y. Shimomura, O. Fourmaux, G-LocON: A P2P-based Communication Framework for Geo-Location Oriented Networks, in: IEICE Information and Communication Technology Forum, Vol. SESSION04, Graz, Austria, 2018, p. SESSION04_3. doi:10.34385/proc.32.SESSION04\_3. URL https://hal .archives-ouvertes.fr/hal-03147512

[129] C. Barberis, G. Malnati, Design and evaluation of a collaborative system for content diffusion and retrieval in vehicular networks, IEEE Transactions on Consumer Electronics 57 (1) (2011) 105-112. doi:10.1109/TCE.2011.5735489.

[130] U. Lee, J.-S. Park, J. Yeh, G. Pau, M. Gerla, Code torrent: Content distribution using network coding in vanet, in: Proceedings of the 1st International Workshop on Decentralized Resource Sharing in Mobile Computing and Networking, MobiShare '06, ACM, New York, NY, USA, 2006, pp. 1-5. doi: 10.1145/1161252.1161254.

URL http://doi.acm.org/10.1145/1161252.1161254

[131] Z. Xie, T.-S. Moh, M. Moh, Asap: Ad-hoc streaming adaptive protocol for supporting p2p streaming over vehicular ad-hoc networks, in: Proceedings of the Annual Southeast Conference, 2013, p. 1-6. doi: $10.1145 / 2498328.2500074$.

[132] Z. Y. Xie, T.-S. Moh, M. Moh, Asap: Ad-hoc streaming adaptive protocol for supporting p2p streaming over vehicular ad-hoc networks, in: Proceedings of the 51st ACM Southeast Conference, ACMSE '13, ACM, New York, NY, USA, 2013, pp. 27:1-27:6. doi:10.1145/2498328.2500074.

URL http://doi.acm.org/10.1145/2498328.2500074

[133] M. S. Almalag, S. Olariu, M. C. Weigle, S. El-Tawab, Peer-to-peer file sharing in vanets using tc-mac, in: 2013 IEEE International Conference on Pervasive Computing and Communications Workshops (PERCOM Workshops), 2013, pp. 84-89. doi:10.1109/PerComW.2013.6529461.

[134] D. T. K. Nataraj B, Realistic mobility model and co-operative peer to peer datatransmission for vanet's using sumo and move, IJREAT International Journal of Research in Engineering and Advanced Technology 3 (2) (2016) 1-6.

[135] K. Eshteiwi, K. Ben Fredj, G. Kaddoum, F. Gagnon, Performance analysis of peer-to-peer v2v wireless communications in the presence of interference, in: 2017 IEEE 28th Annual International Symposium on Personal, Indoor, and Mobile Radio Communications (PIMRC), 2017, pp. 1-6. doi:10.1109/ PIMRC . 2017.8292202.

[136] M. A. Ferrag, A. Ahmim, Esspr: An efficient secure routing scheme based on searchable encryption with vehicle proxy re-encryption for vehicular peer-to-peer social network, Telecommun. Syst. 66 (3) (2017) 481-503. doi:10.1007/s11235-017-0299-y. URL https : //doi.org/10.1007/s11235-017-0299-y 
[137] V. Hassija, V. Chamola, G. Han, J. J. P. C. Rodrigues, M. Guizani, Dagiov: A framework for vehicle to vehicle communication using directed acyclic graph and game theory, IEEE Transactions on Vehicular Technology 69 (4) (2020) 4182-4191. doi:10.1109/TVT.2020.2968494.

[138] T. Wang, Q. Wang, Z. Shen, Z. Jia, Z. Shao, Understanding characteristics and system implications of dag-based blockchain in iot environments, IEEE Internet of Things Journal (2021) 1-1doi:10.1109/ JIOT.2021.3108527.

[139] B. Alaya, Efficient privacy-preservation scheme for securing urban p2p vanet networks, Egyptian Informatics Journal 22 (12 2020). doi:10.1016/j.eij.2020.12.002.

[140] R. Malekian, A. Thakur, L. Nair, C. F. Pedersen, A sensor based peer to peer vehicle data sharing system, an internet of vehicles approach, Journal of Internet Technology 19 (7) (2018) 2155-2162. URL https://jit.ndhu.edu.tw/article/view/1865

[141] B. Beak, M. Zamanipour, K. L. Head, B. Leonard, Peer-to-peer priority signal control strategy in a connected vehicle environment, Transportation Research Record 2672 (18) (2018) 15-26. arXiv: https://doi.org/10.1177/0361198118773567, doi:10.1177/0361198118773567.

URL https://doi.org/10.1177/0361198118773567

[142] Z. You, G. Cheng, Y. Wang, P. Chen, S. Chen, Cross-layer and sdn based routing scheme for p2p communication in vehicular ad-hoc networks, Applied Sciences 9 (22) (2019). doi:10.3390/app9224734. URL https://www.mdpi.com/2076-3417/9/22/4734

[143] Y.-C. Liu, C. Chen, S. Chakraborty, A software defined network architecture for geobroadcast in vanets, in: 2015 IEEE International Conference on Communications (ICC), 2015, pp. 6559-6564. doi:10.1109/ICC.2015.7249370.

[144] B. Dong, W. Wu, Z. Yang, J. Li, Software defined networking based on-demand routing protocol in vehicle ad hoc networks, in: 2016 12th International Conference on Mobile Ad-Hoc and Sensor Networks (MSN), 2016, pp. 207-213. doi:10.1109/MSN.2016.041.

[145] C.-C. Hu, Peer-to-peer data dissemination for deadline-sensitive streaming in vanets, IEEE Access PP (2020) 1-1. doi:10.1109/ACCESS.2020.3011111.

[146] A. Haq, Y. Faheem, A peer-to-peer communication based content distribution protocol for incentiveaware delay tolerant networks, Wireless Networks 26 (01 2020). doi:10.1007/s11276-019-02167-4.

[147] H. Song, Q. Meng, A. Men, P2p computing in design of vanet routing protocol, in: 2007 International Conference on Wireless Communications, Networking and Mobile Computing, 2007, pp. 1502-1507. doi:10.1109/WICOM.2007.379.

[148] C. L. Liu, C. Y. Wang, H. Y. Wei, Mobile chord: Enhancing p2p application performance over vehicular ad hoc network, in: 2008 IEEE Globecom Workshops, 2008, pp. 1-8. doi:10.1109/GLOCOMW. 2008. ECP. 62 .

[149] J. Rybicki, B. Scheuermann, M. Koegel, M. Mauve, Peertis: A peer-to-peer traffic information system, in: Proceedings of the Sixth ACM International Workshop on VehiculAr InterNETworking, VANET '09, ACM, New York, NY, USA, 2009, pp. 23-32. doi:10.1145/1614269.1614275.

URL http://doi.acm.org/10.1145/1614269.1614275

[150] N. Dutta, A peer to peer based information sharing scheme in vehicular ad hoc networks, in: 2010 Eleventh International Conference on Mobile Data Management, 2010, pp. 309-310. doi:10.1109/ MDM. 2010.74.

[151] H.-Y. W. Che-Liang Liu, Chih-Yu Wang, Cross layer mobile chord p2p protocol design for vanet, Int. J. Ad Hoc Ubiquitous Comput. 6 (3) (2010) 150-163. doi:10.1504/IJAHUC. 2010.034968. URL http://dx.doi.org/10.1504/IJAHUC.2010.034968

[152] J. Rybicki, B. Scheuermann, M. Mauve, Peer-to-peer data structures for cooperative traffic information systems, Pervasive and Mobile Computing 8 (2) (2012) 194 - 209, special Issue: Wide-Scale Vehicular Sensor Networks and Mobile Sensing. doi:http://dx.doi.org/10.1016/j.pmcj.2011.06.002. URL http://www.sciencedirect.com/science/article/pii/S1574119211000770

[153] A. Guezzi, A. Lakas, A. Korichi, S. Cherbal, Peer to peer approach based replica and locality awareness to manage and disseminate data in vehicular ad hoc networks, International Journal of Computer Networks and Communications (IJCNC) 12 (2020) 65-81. doi:10.5121/ijcnc.2020.12605. 
[154] H. Hexmoor, S. Tanuja, B. Gupta, Efficient vehicular communication using peer to peer pyramid trees, in: Proceedings of ISCA 34th International Conference on, Vol. 79, 2021, pp. 122-129.

[155] T. Pandey, D. Garg, M. Gore, Structured p2p overlay of mobile brokers for realizing publish/subscribe communication in vanet, TheScientificWorldJournal 2014 (2014) 1-12.

[156] N.-M. Balouchzahi, M. Fathy, A. Akbari, An efficient infrastructure based service discovery in vehicular networks using p2p structures, The Journal of Supercomputing 72 (3) (2016) 1013-1034. doi:10. $1007 /$ s11227-016-1638-6.

URL https://doi.org/10.1007/s11227-016-1638-6

[157] A. Garg, K. Pandey, B. Singh, Hierarchical map-based location service for vanets in urban environments, in: 2014 Seventh International Conference on Contemporary Computing (IC3), 2014, pp. 199-205. doi:10.1109/IC3.2014.6897173.

[158] J. M. Jeon, C. S. Hong, Scalable private p2p network for distributed and hierarchical machine learning in vanets, in: 2021 International Conference on Information Networking (ICOIN), 2021, pp. 627-629. doi:10.1109/ICOIN50884.2021.9333988.

[159] H.-C. Jang, T.-Y. Hsu, Infrastructure based chord structure for $\mathrm{p} 2 \mathrm{p}$ file sharing over vehicular network, Journal of Networks 8 (3) (2013) 588 - 597.

[160] W. Chen, S. Cai, Ad hoc peer-to-peer network architecture for vehicle safety communications, IEEE Communications Magazine 43 (4) (2005) 100-107. doi:10.1109/MCOM. 2005.1421912.

[161] T. Atéchian, Z. Torbey, N. Bennani, L. Brunie, Coffee: Cooperative and infrastructure-free peerto-peer system for vanet, in: 2009 9th International Conference on Intelligent Transport Systems Telecommunications, (ITST), 2009, pp. 510-515. doi:10.1109/ITST.2009.5399300.

[162] H. Perkuhn, I. El-Khayat, Geo-supported p2p vanet for its applications, in: Proceedings of the 5th International Conference on Principles, Systems and Applications of IP Telecommunications, IPTcomm '11, ACM, New York, NY, USA, 2011, pp. 6:1-6:2. doi:10.1145/2124436.2124444.

URL http://doi.acm.org/10.1145/2124436.2124444

[163] N. Ahmadifard, H. Nabizadeh, M. Abbaspour, Iseff: An id-based scalable and efficient distributed file sharing technique in vehicular ad hoc networks, Wireless Personal Communications 75 (2) (2014) 821-841. doi:10.1007/s11277-013-1393-4. URL https://doi.org/10.1007/s11277-013-1393-4

[164] A. Valibak, M. Fathy, N. Balouchzahi, A. Akbari, A two-purpose peer-to-peer structure for vehicle navigating and traffic information disseminating in vehicular networks, in: 2014 4th International Conference on Computer and Knowledge Engineering (ICCKE), 2014, pp. 316-321. doi:10.1109/ ICCKE. 2014.6993334.

[165] M. Picone, S. Busanelli, M. Amoretti, F. Zanichelli, G. Ferrari, Advanced Technologies for Intelligent Transportation Systems, Vol. 139, Springer International Publishing, 2015.

[166] S. Azizi, M. Khalili, M. Robatmily, A new method to enhance the efficiency of peer-to-peer vehicular networks, in: 2015 2nd International Conference on Knowledge-Based Engineering and Innovation (KBEI), 2015, pp. 993-997. doi:10.1109/KBEI.2015.7436180.

[167] K. R. M. F. Ali Asghar Valibak, Nik Mohammad Balouchzahi, A. Akbari, An optimized two-purpose peer-to-peer vanet to disseminate traffic information and reduce travel time, International Journal of Future Computer and Communication 4 (2) (2015) 1-6.

[168] R. I. Meneguette, A. Boukerche, Peer-to-peer protocol for allocated resources in vehicular cloud based on v2v communication, in: 2017 IEEE Wireless Communications and Networking Conference (WCNC), 2017, pp. 1-6. doi:10.1109/WCNC.2017.7925602.

[169] A. Pandey, U. Kumar, H. Sharma, S. S. Arkatkar, p2ptraf: An open library implementing p2p vehicular network for preliminary real-field experiments, in: 2021 International Conference on COMmunication Systems NETworkS (COMSNETS), 2021, pp. 753-758. doi:10.1109/COMSNETS51098. 2021. 9352920.

[170] S. H. Lin, J. Y. Hu, C. F. Chou, I. C. Chang, C. C. Hung, A novel social cluster-based p2p framework for integrating vanets with the internet, in: 2009 IEEE Wireless Communications and Networking Conference, 2009, pp. 1-6. doi:10.1109/WCNC. 2009.4917531. 
[171] S. L. Tsao, C. M. Cheng, Design and evaluation of a two-tier peer-to-peer traffic information system, IEEE Communications Magazine 49 (5) (2011) 165-172. doi:10.1109/MCOM.2011.5762814.

[172] J. Guo, C. Hu, Y. Huo, Y. Liu, L. Zhang, Information sharing service in vanets with enhanced twotier p2p communications, in: The 15th International Symposium on Wireless Personal Multimedia Communications, 2012, pp. 65-69.

[173] J.-S. L. C.-G. Liu, A novel group-based location service management scheme in vanets, Journal of Advances in Computer Networks 1 (1) (2013) 23 - 28. doi:10.7763/JACN. 2013.V1.5. URL www.jacn.net/papers/5-C008.pdf

[174] K. Lu, C. M. Cheng, S. L. Tsao, An adaptive routing algorithm for two-tier traffic information system, in: 2012 IEEE Consumer Communications and Networking Conference (CCNC), 2012, pp. 311-315. doi:10.1109/CCNC. 2012.6181108.

[175] C. M. Cheng, A two-tier vanet/p2p system for information retrieval in vehicular environments, in: 2013 IEEE International Conference on Pervasive Computing and Communications Workshops (PERCOM Workshops), 2013, pp. 415-416. doi:10.1109/PerComW.2013.6529529.

[176] C. M. Cheng, S. L. Tsao, Adaptive lookup protocol for two-tier vanet/p2p information retrieval services, IEEE Transactions on Vehicular Technology 64 (3) (2015) 1051-1064. doi:10.1109/TVT. 2014.2329015.

[177] Y. Aoki, T. Ohta, Y. Kakuda, Experimental evaluation of information dissemination scheme based on autonomous clustering and p2p network in vanets, in: 2015 Third International Symposium on Computing and Networking (CANDAR), 2015, pp. 275-278. doi:10.1109/CANDAR.2015.47.

[178] V. Anjali, P. Raghavendra, P. Arun, T. Rajeev, Information retrieval in two-tier vanet/p2p using rsu as a superpeer, Wireless Communication Technology 2 (2018) 1-9. doi:10.18063/wct.v2i1.456.

[179] Y. H. Lee, C. R. Dow, L. H. Huang, Y. C. Lin, S. F. Hwang, W. B. Lee, An efficient geo-aware peer-to-peer resource discovery and sharing scheme in vehicular ad-hoc networks, in: 2012 Ninth International Conference on Information Technology - New Generations, 2012, pp. 54-59. doi:10. 1109/ITNG. 2012.16.

[180] O. S. Oubbati, M. Atiquzzaman, P. Lorenz, M. H. Tareque, M. S. Hossain, Routing in flying ad hoc networks: Survey, constraints, and future challenge perspectives, IEEE Access 7 (2019) 81057-81105.

[181] P. Louridas, C. Ebert, Machine learning, IEEE Software 33 (5) (2016) 110-115. doi:10.1109/MS. 2016.114.

[182] A. Shrestha, A. Mahmood, Review of deep learning algorithms and architectures, IEEE Access 7 (2019) 53040-53065. doi:10.1109/ACCESS . 2019.2912200.

[183] I. F. Akyildiz, A. Kak, S. Nie, 6g and beyond: The future of wireless communications systems, IEEE Access 8 (2020) 133995-134030. doi:10.1109/ACCESS.2020.3010896.

[184] S. Nath, A. Paul, R. Banerjee, S. Bhaumik, J. K. Sing, S. Kumar Sarkar, Optimizing fanet routing using a hybrid approach of firefly algorithm and aco-lévy flight, in: 2020 IEEE VLSI DEVICE CIRCUIT AND SYSTEM (VLSI DCS), 2020, pp. 378-383. doi:10.1109/VLSIDCS47293.2020.9179956.

[185] I. Bekmezci, I. Sen, E. Erkalkan, Flying ad hoc networks (fanet) test bed implementation, in: 2015 7th International Conference on Recent Advances in Space Technologies (RAST), 2015, pp. 665-668. doi:10.1109/RAST . 2015.7208426.

[186] S. Raj, V. K. Panchal, P. C. Vashist, R. Chopra, Fanets: Current trends and challenges, in: 2019 2nd International Conference on Power Energy, Environment and Intelligent Control (PEEIC), 2019, pp. 472-475. doi:10.1109/PEEIC47157.2019.8976730.

[187] S. Raj, V. K. Panchal, P. C. Vashist, R. Chopra, Fanets: Current trends and challenges, in: 2019 2nd International Conference on Power Energy, Environment and Intelligent Control (PEEIC), 2019, pp. 472-475. doi:10.1109/PEEIC47157.2019.8976730.

[188] W. Qiang, Z. Zhongli, Reinforcement learning model, algorithms and its application, in: 2011 International Conference on Mechatronic Science, Electric Engineering and Computer (MEC), 2011, pp. 1143-1146. doi:10.1109/MEC.2011.6025669.

[189] S. S. Doddalinganavar, P. V. Tergundi, R. S. Patil, Survey on deep reinforcement learning protocol in vanet, in: 2019 1st International Conference on Advances in Information Technology (ICAIT), 2019, 
pp. 81-86. doi:10.1109/ICAIT47043.2019.8987282.

[190] Arun K., Rejimoan R., A survey on network path identification using bio inspired algorithms, in: 2016 2nd International Conference on Advances in Electrical, Electronics, Information, Communication and Bio-Informatics (AEEICB), 2016, pp. 387-389. doi:10.1109/AEEICB . 2016.7538314.

[191] P. T. Daely, D. Kim, Bio-inspired cooperative localization in industrial wireless sensor network, in: 2019 15th IEEE International Workshop on Factory Communication Systems (WFCS), 2019, pp. 1-4. doi : 10.1109/WFCS. 2019.8758004.

[192] S. Bitam, A. Mellouk, S. Zeadally, Bio-inspired routing algorithms survey for vehicular ad hoc networks, IEEE Communications Surveys Tutorials 17 (2) (2015) 843-867. doi:10.1109/COMST.2014. 2371828.

[193] M. Bahrami, Cloud computing for emerging mobile cloud apps, in: 2015 3rd IEEE International Conference on Mobile Cloud Computing, Services, and Engineering, 2015, pp. 4-5. doi:10.1109/ MobileCloud.2015.40.

[194] N. K. Giang, R. Lea, M. Blackstock, V. C. M. Leung, Fog at the edge: Experiences building an edge computing platform, in: 2018 IEEE International Conference on Edge Computing (EDGE), 2018, pp. 9-16. doi:10.1109/EDGE. 2018.00009.

[195] S. Singla, N. K. Bhati, S. Aswath, Future Opportunistic Fog/Edge Computational Models and their Limitations, Wiley, 2021, Ch. 2, pp. 27-46. doi:10.1002/9781119670087.ch2.

[196] X. Zhang, X. Chen, Data security sharing and storage based on a consortium blockchain in a vehicular ad-hoc network, IEEE Access 7 (2019) 58241-58254. doi:10.1109/ACCESS .2018.2890736.

[197] L. Xie, Y. Ding, H. Yang, X. Wang, Blockchain-based secure and trustworthy internet of things in sdn-enabled 5g-vanets, IEEE Access 7 (2019) 56656-56666. doi:10.1109/ACCESS.2019. 2913682.

[198] H. Kaushal, V. Jain, S. Kar, Overview of Wireless Optical Communication Systems, Springer India, 2017, Ch. 1, pp. 1 - 39. doi:10.1007/978-81-322-3691-7_1.

[199] M. Z. Chowdhury, M. T. Hossan, A. Islam, Y. M. Jang, A comparative survey of optical wireless technologies: Architectures and applications, IEEE Access 6 (2018) 9819-9840.

[200] W. Liu, J. Ding, J. Zheng, X. Chen, C. L. I, Relay-assisted technology in optical wireless communications: A survey, IEEE Access 8 (2020) 194384-194409. doi:10.1109/ACCESS.2020.3031288.

[201] A. Vater, Madpastry : A dht substrate for practicably sized manets, in: in Proc. of 5th Workshop on Applications and Services in Wireless Networks (ASWN2005), 2010, pp. 1-12.

[202] W. Anwar, N. Franchi, G. Fettweis, Physical layer evaluation of v2x communications technologies: 5g nr-v2x, lte-v2x, ieee 802.11bd, and ieee 802.11p, in: 2019 IEEE 90th Vehicular Technology Conference (VTC2019-Fall), 2019, pp. 1-7. doi:10.1109/VTCFall.2019.8891313.

[203] G. Naik, B. Choudhury, J.-M. Park, Ieee 802.11bd amp; $5 \mathrm{~g}$ nr v2x: Evolution of radio access technologies for v2x communications, IEEE Access 7 (2019) 70169-70184. doi:10.1109/ACCESS. 2019. 2919489.

[204] W. Anwar, N. Franchi, G. Fettweis, Physical layer evaluation of v2x communications technologies: $5 \mathrm{~g}$ nr-v2x, lte-v2x, ieee 802.11bd, and ieee 802.11p, in: 2019 IEEE 90th Vehicular Technology Conference (VTC2019-Fall), 2019, pp. 1-7. doi:10.1109/VTCFall.2019.8891313.

[205] A. Ghosh, A. Maeder, M. Baker, D. Chandramouli, 5g evolution: A view on 5g cellular technology beyond 3gpp release 15, IEEE Access 7 (2019) 127639-127651. doi:10.1109/ACCESS. 2019. 2939938.

[206] Y. Liu, X. Yuan, Z. Xiong, J. Kang, X. Wang, D. Niyato, Federated learning for 6g communications: Challenges, methods, and future directions, China Communications 17 (9) (2020) 105-118. doi: 10.23919/JCC. 2020.09.009.

[207] G. Wikström, J. Peisa, P. Rugeland, N. Johansson, S. Parkvall, M. Girnyk, G. Mildh, I. L. Da Silva, Challenges and technologies for 6g, in: 2020 2nd 6G Wireless Summit (6G SUMMIT), 2020, pp. 1-5. doi:10.1109/6GSUMMIT49458.2020.9083880.

[208] S. Wang, S. Lu, Q. Zhang, Instantly decodable network coding-assisted data dissemination for prioritized services in vehicular ad hoc networks, International Journal of Distributed Sensor Networks 15 (4) (2019) 1550147719842137. arXiv:https://doi.org/10.1177/1550147719842137, doi: $10.1177 / 1550147719842137$. 
URL https://doi.org/10.1177/1550147719842137

[209] D. Vasiliev, A. Chunaev, A. Abilov, I. Kaysina, D. Meitis, Application layer arq and network coding for qos improving in uav-assisted networks, in: 2019 25th Conference of Open Innovations Association (FRUCT), 2019, pp. 353-360. doi:10.23919/FRUCT48121.2019.8981502.

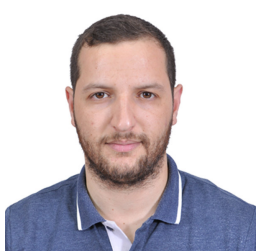

Abdelkader Ilyes Ameur is a PhD student in Computer Science. He received his degree of Engineer (2008) and the M.Sc. degree (2012) in computer science from the University of Laghouat, Algeria. He is currently working as an Assistant Lecturer and Research Assistant in the Computer Science and Mathematics Lab (LIM) at the University of Laghouat. His research interests fall into the areas of wireless networking, UAVs, artificial intelligence, and mobile networks.

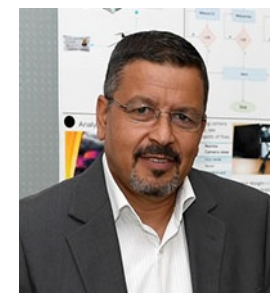

Abderrahmane Lakas received his MS (1990) and PhD (1996) in Computer Systems from the University of Paris VI, Paris, France. He joined the College of Information Technology, UAE University in 2003. He is teaching various courses on computer networks and network security. Dr. Lakas had many years of industrial experience holding various technical positions in telecommunication companies such as Netrake (Plano, Texas, 2002), Nortel (Ottawa, 2000) and Newbridge (Ottawa, 1998). He spent two years (94-96) as a Research Associate at the University of Lancaster, UK. Dr. Lakas has been conducting research in the areas of network design and performance, voice over IP, quality of service and wireless networks. He is a senior member of the IEEE and IEEE Communications Society. Dr. Lakas is in the editorial board of Journal of Communications (Actapress), and Journal of Computer Systems, Networks, and Communications (Hindawi). He is serving on the technical program committees of many international conferences GLOBCOM, ICC, VTC, etc.

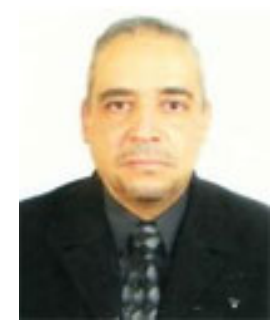

Mohamed Bachir Yagoubi is currently a Professor at University of Laghouat, Algeria. He received his MSc in 1992 from Paris 13 University, Paris, France and the $\mathrm{PhD}$ degree in Computer Science in 1997 from Evry-val-d'Essonne University, France. His research interests include Distributed Systems, Fault tolerance, Security, and Vehicular Networks. 


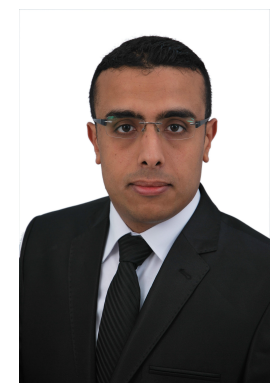

Omar Sami Oubbati is an Associate Professor at University Gustave Eiffel in the region of Paris, France. He is a member of the Gaspard Monge Computer Science laboratory (LIGM CNRS UMR 8049). He received his degree of Engineer (2010), M.Sc. in Computer Engineering (2011), M.Sc. degree (2014), and a PhD in Computer Science (2018), all from University of Laghouat, Algeria. From Oct. 2016 to Oct. 2017, he was a Visiting PhD Student with the Laboratory of Computer Science, University of Avignon, France. He spent 6 years as an Assistant Professor at the Electronics department, University of Laghouat, Algeria and a Research Assistant in the Computer Science and Mathematics Lab (LIM) at the same university. His main research interests are in Flying and Vehicular ad hoc networks, Energy harvesting and Mobile Edge Computing, Energy efficiency and Internet of Things (IoT). He is the recipient of the 2019 Best Survey Paper for Vehicular Communications (Elsevier). He has actively served as a reviewer for flagship IEEE Transactions journals and conferences, and participated as a Technical Program Committee Member for a variety of international conferences, such as IEEE ICC, IEEE CCNC, IEEE ICCCN, IEEE WCNC, IEEE ICAEE, and IEEE ICAIT. He serves on the editorial board of Vehicular Communications Journal of Elsevier and Communications Networks Journal of Frontiersin. He has also served as guest editor for a number of international journals. He is a member of the IEEE and IEEE Communications Society. 